\title{
DECAY ESTIMATES FOR BI-SCHRÖDINGER OPERATORS IN DIMENSION ONE
}

\author{
AVY SOFFER, ZHAO WU AND XIAOHUA YAO ${ }^{\dagger}$
}

\begin{abstract}
This paper is devoted to study the time decay estimates for bi-Schrödinger operators $H=\Delta^{2}+V(x)$ in dimension one with decaying potentials $V(x)$. We first deduce the asymptotic expansions of resolvent of $H$ at zero energy threshold without/with the presence of resonances, and then characterize these resonance spaces corresponding to different types of zero resonance in suitable weighted spaces $L_{s}^{2}(\mathbf{R})$. Next we use them to establish the sharp $L^{1}-L^{\infty}$ decay estimates of Schrödinger groups $e^{-i t H}$ generated by bi-Schrödinger operators also with zero resonances. As a consequence, Strichartz estimates are obtained for the solution of fourth-order Schrödinger equations with potentials for initial data in $L^{2}(\mathbf{R})$. In particular, it should be emphasized that the presence of zero resonances does not change the optimal time decay rate of $e^{-i t H}$ in dimension one, except at requiring faster decay rate of the potential.
\end{abstract}

\section{INTRODUCTION}

1.1. Backgrounds and problems. The higher-order elliptic operators $P(D)+V$ have been extensively considered as general Hamiltonian operator by many people in different contexts. We just mention some references, not all. For instance, see Schechter [Sch71] for spectral theory, Kuroda Kur78, Agmon Agm75, Hörmander [Hö05] for scattering theory, Davies [Dav97, Davies and Hinz DH98b], Deng et al [DDY14] for the Gaussian estimates of semigroup, and as well [HS15, HS17, BS91, Mou81, SYY18, for other interesting topics.

In this paper, we will consider the time decay estimates for the bi-Schrödinger operator:

$$
H=\Delta^{2}+V(x), H_{0}=\Delta^{2},
$$

where $V(x)$ is a real-valued function satisfying $|V(x)| \lesssim(1+|x|)^{-\beta}$ for some $\beta>0$. Such investigations are motivated in large part by classical Schrödinger operator $-\Delta+V$ and their fundamental applications. In the last thirty years, the dispersive estimates of Schrödinger operator have been one of the key topics, which were applied broadly to nonlinear Schrödinger equations, see e.g. [Bou91, Caz03, Tao06, JSS91, KT98, NaSc11, Sch07, Sch20, Sim18a, Sim18b] and references therein.

For the free propagators $e^{-i t H_{0}}$ and $H_{0}=\Delta^{2}$, Ben-Artzi, Koch and Saut in [BKS] have proved the following pointwise estimates for the kernel of $e^{-i t H_{0}}$ on $\mathbf{R}^{n}$ :

$$
\left|\partial_{x}^{\alpha} I_{0}(x, t)\right| \lesssim t^{-\frac{n+|\alpha|}{4}}\left(1+\frac{|x|}{t^{1 / 4}}\right)^{-\frac{(n-|\alpha|)}{3}}, x \in \mathbf{R}^{n},
$$

where $I_{0}(x, t)$ be the kernel of $e^{-i t H_{0}}$ and $\alpha \in \mathbf{N}^{n}$, which immediately implies the following $L^{1}-L^{\infty}$ estimates:

$$
\left\|\partial^{\alpha} e^{-i t H_{0}}\right\|_{L^{1}\left(\mathbf{R}^{n}\right) \rightarrow L^{\infty}\left(\mathbf{R}^{n}\right)} \lesssim|t|^{-\frac{n+|\alpha|}{4}}, \quad|\alpha| \leq n .
$$

Date: December 16, 2021.

Key words and phrases. Decay estimates, Bi-Schrödinger operators, Zero resonances, Resolvent expansions.

$\dagger$ Corresponding author. 
Moreover, we notice that the regular term $\partial^{\alpha}$ of the inequality (1.3) can be replaced by fractional power $H_{0}^{\frac{s}{4}}$ for any $0 \leq s \leq n$ ( see e.g. CMY12 ).

For the fourth order Schrödinger operators $H=\Delta^{2}+V(x)$, such time decay estimates were much less understood than Schrödinger operators. Recently, Feng, Soffer and Yao [FSY18] deduced the asymptotic expansion of the resolvent $R_{V}(z)=(H-z)^{-1}$ around zero threshold, assuming that zero is a regular point of $H$ for $n \geq 5$ and $n=3$, and showed that in the regular case, the KatoJensen time decay estimate of $e^{-i t H}$ is $(1+|t|)^{-n / 4}$ for $n \geq 5$ and the $L^{1}-L^{\infty}$ decay estimates is $O\left(|t|^{-1 / 2}\right.$ ) for $n=3$ (not optimal).

Based on the higher energy estimates of resolvent in [FSY18], Erdogăn, Green and Toprak in [EGT21] for $n=3$ and Green-Toprak in [GT19] for $n=4$ further derived the asymptotic expansion of $R_{V}(z)$ near zero with the presence of resonance or eigenvalue, and obtained $L^{1}-L^{\infty}$ estimates for each kind of zero resonances. They proved the $L^{1}-L^{\infty}$ time decay estimate is $|t|^{-n / 4}$ for $n=3,4$ if zero is a regular point, and zero energy resonance will change the time decay rate.

Moreover, we mention that Kato-Jensen estimates and asymptotic expansion of resolvent have been established by [FSWY20] for poly-Schrödinger operators $(-\Delta)^{m}+V$ with $n \geq 2 m \geq 4$. In previous works, Murata in [Mur82] have generalized Kato and Jensen's work [JK79] to a certain class of $P(D)+V$ assume that $P(D)$ satisfies

$$
(\nabla P)\left(\xi_{0}\right)=0,\left.\quad \operatorname{det}\left[\partial_{i} \partial_{j} P(\xi)\right]\right|_{\xi_{0}} \neq 0 .
$$

However, the poly-harmonic operators $H_{0}=(-\Delta)^{m}$ do not satisfy the nondegenerate condition (1.4) at zero but the case $m=1$. Hence the results in [FSWY20, were not covered by Mur82]. In particular, the degeneracy of $(-\Delta)^{m}$ at zero energy certainly leads to more complicated zero resonances classifications and asymptotic expansions of the resolvent for $(-\Delta)^{m}+V$ as $m \geq 2$. This is actually one of the main difficult points for the higher order estimates.

In this paper, we are devoted to establishing the same $L^{1}-L^{\infty}$ estimates of $H=\Delta^{2}+V$ (with regularity terms) as the free estimates (1.3) in dimension one. We showed that the presence of zero resonance of $H$ does not affect the time decay rate of $e^{-i t H}$ but requires higher decay rate of potential $V$. This seems to be distinct phenomena than other situations ( see e.g. [FSWY20, EGT21, GT19, Sch20]).

As a consequence, Strichartz estimates were obtained for the fourth-order Schrodinger equations with potentials for initial data in $L^{2}(\mathbf{R})$. Furthermore, by using similar methods, Li, Soffer and Yao [LSY21] have derived the $L^{1}-L^{\infty}$ estimates for $H$ with zero resonances in two dimensional case. In particular, we remark that arguments used here can be applied to the higher-order elliptic operators $(-\Delta)^{m}+V$.

For Schrödinger operator $-\Delta+V$, the $L^{1}-L^{\infty}$ decay estimates have been widely investigated by many people. For $n \geq 3$, Journé, Soffer and Sogge in [JSS91] first established the $L^{1}-L^{\infty}$ dispersive estimate in the regular case. For $n \leq 3$, one can see Weder [Wed00, Rodnianski and Schlag [RS04, Schlag and Goldberg [GS04, Schlag [Sch05] and so on. Yajima in Yaj95 has also established the $L^{1}-L^{\infty}$ estimates for $-\Delta+V$ by wave operator method. For many further studies, one can refer to Wed99, ES04, ES06, GG15, GG17, Sch07, Sch20] and therein references.

1.2. Some notations. In this subsection, we collect some notations used throughout the paper.

- For $a \in \mathbf{R}$, we write $a \pm$ to denote $a \pm \epsilon$ for any small $\epsilon>0$. [a] denotes the largest integer less than or equal to $a$. For $a, b \in \mathbf{R}^{+}$, we denote $A \lesssim B$ (or $B \gtrsim A$ ) which means that there exists some constant $c>0$ such that $A \leq c B$ (or $B \geq c A$ ). Moreover, if $A \lesssim B$ and $B \lesssim A$, then we write $A \sim B$. 
- For $s, s^{\prime} \in \mathbf{R}, B\left(s, s^{\prime}\right)$ denote the space of the bounded operators from $L_{s}^{2}(\mathbf{R})$ to $L_{s^{\prime}}^{2}(\mathbf{R})$, where $L_{s}^{2}(\mathbf{R})$ is the weighted $L^{2}$ space:

$$
L_{s}^{2}(\mathbf{R})=\left\{f \in L_{l o c}^{2}(\mathbf{R}):(1+|\cdot|)^{s} f \in L^{2}(\mathbf{R})\right\} .
$$

1.3. Main Results. In the subsection, we will state our main results. Recall that, if there exists a nonzero solution $\phi(x) \in L^{2}(\mathbf{R})$ satisfying the equation $H \phi(x)=0$ in distributional sense, then we say that zero is an eigenvalue of $H=\Delta^{2}+V(x)$. In general, there may exist a nonzero resonant solution $\phi(x)$ in $L_{-s}^{2}(\mathbf{R})$ with some $s>0$ such that $H \phi(x)=0$, which usually results in the obstructions to dispersive estimates at low energy. In the present case, we first introduce the zero resonance definitions of $\Delta^{2}+V(x)$ in dimension one. For $\sigma \in \mathbf{R}$, let $W_{\sigma}(\mathbf{R})$ denotes the intersection space $W_{\sigma}(\mathbf{R})=\bigcap_{s>\sigma} L_{-s}^{2}(\mathbf{R})$. Note that, $W_{\sigma_{1}}(\mathbf{R}) \supset W_{\sigma_{2}}(\mathbf{R})$ if $\sigma_{1}>\sigma_{2}$ and $W_{0}(\mathbf{R}) \supset L^{2}(\mathbf{R})$.

Definition 1.1. Let $H=\Delta^{2}+V$ on $L^{2}(\mathbf{R})$ and $|V(x)| \lesssim(1+|x|)^{-\beta}$ for some $\beta>0$. Then we say that

(i) zero is a first kind resonance of $H$ if there exists some nonzero $\phi(x) \in W_{\frac{3}{2}}(\mathbf{R})$ but no any nonzero $\phi \in W_{\frac{1}{2}}(\mathbf{R})$ such that $H \phi(x)=0$ in the distributional sense;

(ii) zero is a second kind resonance of $H$ if there exists some nonzero $\phi(x) \in W_{\frac{1}{2}}(\mathbf{R})$ but no any nonzero $\phi \in L^{2}(\mathbf{R})$ such that $H \phi(x)=0$ in the distributional sense;

(iii) zero is an eigenvalue of $H$ if there exists some nonzero $\phi(x) \in L^{2}(\mathbf{R})$ such that $H \phi(x)=0$ in the distributional sense;

(iv) zero is a regular point of $H$ if zero is neither a resonance nor an eigenvalue of $H$.

Remark 1.2. Some examples of zero resonance and zero eigenvalue:

(i) (Zero resonance) Assume that $\phi \in C^{\infty}(\mathbf{R})$ is a positive function which is equal to $c|x|+d$ for $|x|>1$. Then $H \phi(x)=0$ when $V(x)=-\left(\Delta^{2} \phi\right) / \phi(x)$. One can easily check that $V(x) \in C_{0}^{\infty}(\mathbf{R})$ and $\phi(x) \in W_{\frac{3}{2}}(\mathbf{R})$. Hence by Definition 1.1, we know that the zero is a resonance point of $H$ even with a compactly supported smooth potential.

(ii) (Zero eigenvalue) Unlike zero resonance, the zero eigenvalue problem of $H$ is subtler. For instance, let $\phi(x)=\left(1+x^{2}\right)^{-s}$ with $s>\frac{1}{4}$ and $V(x)=-\left(\Delta^{2} \phi\right) / \phi(x)$. Then one has that

$$
V(x)=O\left((1+|x|)^{-4}\right), \quad|x| \rightarrow \infty,
$$

and $\phi \in L^{2}(\mathbf{R})$ satisfying $H \phi=0$. This means that zero is an eigenvalue of $H$ with such slowly decaying potential.

On the other hand, it was pointed out to us by Prof. Goldberg Gold21 that if a potential $V(x)$ has faster decay, then zero eigenvalue of the one-dimensional bi-Schrödinger operator may not occur, see e.g.[DT79] for Schrödinger operator case. In fact, if the potential $V$ satisfies the decay condition of Theorem 1.3 below, then a proof on the absence of zero eigenvalue has been stated in Lemma 3.5 of Section 3 .

Now we state the first main result under the decay conditions depending on the types of zero energy defined above. In this paper due to the faster decay rate of $V$ and the absence of zero eigenvalue, we do not necessarily discuss zero eigenvalue case in Theorem 1.3 below. Even so, it should be noticed that compared with the Schrödinger operator $-\Delta+V$ on $L^{2}(\mathbf{R})$ ( see e.g. Jensen and Nenciu [JN01, JN04] ), the classification of the zero resonance for $H=\Delta^{2}+V$ in one dimensional case is more complicated due to the low dimensionality (i.e. $n=1$ ) and the degeneracy of $\Delta^{2}$ at zero energy ( i.e. zero is the degenerate critical point of the symbol $\xi^{4}$ ); it 
leads to the more singular terms in the zero asymptotic expansion of resolvent $R_{V}^{ \pm}(\mu)$ ( see Theorem 1.8 below or Section 3 ).

Theorem 1.3. 1 Let $|V(x)| \lesssim(1+|x|)^{-\beta}$ with some $\beta>0$ depending on the following types:

$$
\beta>\left\{\begin{array}{l}
13, \quad \text { zero is the regular point, } \\
17, \text { zero is the first kind resonance, } \\
25, \quad \text { zero is the second kind resonance. }
\end{array}\right.
$$

Assume that $H=\Delta^{2}+V$ has no positive embedded eigenvalues and $P_{a c}(H)$ denotes the projection onto the absolutely continuous spectrum space of $H$. Then we have the following decay estimate:

$$
\left\|e^{-i t H} P_{a c}(H)\right\|_{L^{1}(\mathbf{R}) \rightarrow L^{\infty}(\mathbf{R})} \lesssim|t|^{-\frac{1}{4}}, \quad t \neq 0 .
$$

Furthermore, for any $0 \leq \alpha<1$, the following improved estimate holds:

$$
\left\|H^{\frac{\alpha}{4}} e^{-i t H} P_{a c}(H)\right\|_{L^{1}(\mathbf{R}) \rightarrow L^{\infty}(\mathbf{R})} \lesssim|t|^{-\frac{1+\alpha}{4}}, \quad t \neq 0 .
$$

As we will see later, the needed decay rates of the potential $V$ mainly depend on the lower energy expansions of resolvent $R_{V}(z)$ for different zero resonance types (see Theorem 1.8 below). According to Remark 1.10 after Theorem 1.8, we still can obtain the decay estimates (1.6) and (1.7) by using the weak expansions of $R_{V}(z)$ under the assumption of the following less decay rates:

$$
\beta> \begin{cases}7, & \text { zero is the regular point, } \\ 9, & \text { zero is the first kind resonance, } \\ 13, & \text { zero is the second kind resonance. }\end{cases}
$$

In the paper, we do not follow the way along with these interesting improvements. Instead, we focus on the simplicity of the proof based on the expansions of resolvent $R_{V}(z)$ in Theorem 1.8 , Noting that the estimates (1.6) and (1.7) above are optimal in view of the free estimate (1.3) with $V=0$.

In particular, the optimal time decay rate ( e.g. $|t|^{-\frac{1}{4}}$ in the (1.6) ) does not change as the zero resonance arises, but only requires the faster decay rate $\beta$ of potential $V$ as the compensations of zero singularities. Moreover, note that for Schrödinger operator $-\Delta+V$, Egorova, Kopylova, Marchenko and Teschl in [EKMT16] have proved that the resonant case does not need additional decay of the potential, hence it is an interesting question whether we can show that the sharp decay estimates (1.6) still hold on the same decay condition on $V(x)$ for $H=\Delta^{2}+V$.

Moreover, in the above Theorem 1.3, we also assume that $H=\Delta^{2}+V$ has no positive eigenvalues embedded in the absolutely continuous spectrum, which has been the indispensable condition in dispersive estiamtes. For Schrödinger operator $-\Delta+V$, Kato in Kat59 has shown the absence of positive eigenvalues for $H=-\Delta+V$ with the decay potentials $V=o\left(|x|^{-1}\right)$ as $|x| \rightarrow \infty$. For the bi-Schrödinger operator $H=\Delta^{2}+V$, the situation seems to be more complicated than the second order operator, since there exist examples even with compactly supported smooth potentials such that positive eigenvalues appear ( see e.g. [FSWY20] ).

However, interestingly, a simple criterion has been proved in [FSWY20] that $H=\Delta^{2}+V$ has no any positive eigenvalues under the assumption that the potential $V$ is bounded and satisfies the repulsive condition (i.e. $(x \cdot \nabla) V \leq 0)$. In particular, such criterion also works for the general higher order elliptic operator $P(D)+V$. We also notice that for a general selfadjoint operator $\mathcal{H}$ on $L^{2}\left(\mathbf{R}^{n}\right)$, even if $\mathcal{H}$ has a simple embedded eigenvalue, Costin and Soffer in [CS01] have proved

\footnotetext{
${ }^{1}$ After the submission of our paper, we learned that by a different method, Thomas Hill has proved that the estimates (1.6) and (1.7) hold, assuming that zero is a regular point for $H=\Delta^{2}+V$ with a compactly supported potential $V(x) \in C_{c}^{1}(\mathbf{R})$, see his dissertation Hill20.
} 
that $\mathcal{H}+\epsilon W$ can kick off the eigenvalue located in a small interval under certain small perturbation of potential.

As an interesting consequence of $L^{1}-L^{\infty}$ estimates above, by Keel-Tao [KT98] method, we immediately obtain Strichartz estimate to the fourth-order Schrödinger equation on $\mathbf{R}$ :

$$
\left\{\begin{array}{l}
i \partial_{t} \phi=\left(\Delta^{2}+V\right) \phi+F(t, x), \quad(t, x) \in \mathbf{R}^{1+1}, \\
\phi(0, \cdot)=\phi_{0}(x) .
\end{array}\right.
$$

Recall that, a pair $(q, r)$ is said to be $\sigma$-admissible if

$$
\frac{1}{q}+\frac{\sigma}{r}=\frac{\sigma}{2}, \quad 2 \leq q \leq \infty, \quad(q, r, \sigma) \neq(2, \infty, 1) .
$$

Corollary 1.4. Let $V$ and $H=\Delta^{2}+V$ satisfy the same conditions as Theorem 1.3 above. Then for any $\frac{1}{4}$-admissible pairs $(q, r)$ and $(\tilde{q}, \tilde{r})$, we have the following estimates:

(i) Homogeneous Strichartz Estimate

$$
\left\|e^{-i t H} P_{a c}(H) \phi_{0}\right\|_{L_{t}^{q} L_{x}^{r}(\mathbf{R} \times \mathbf{R})} \lesssim\left\|\phi_{0}\right\|_{L^{2}(\mathbf{R})} .
$$

(ii) Dual Homogeneous Strichartz Estimate

$$
\left\|\int_{\mathbf{R}} e^{-i s H} P_{a c}(H) F(s, \cdot) d s\right\|_{L_{x}^{2}(\mathbf{R})} \lesssim\|F\|_{L_{t}^{\tilde{q}^{\prime}} L_{x}^{\tilde{r}^{\prime}}(\mathbf{R} \times \mathbf{R})} .
$$

(iii) The solution $\phi(t, x)$ of the equation (1.8) satisfies

$$
\left\|P_{a c}(H) \phi\right\|_{L_{t}^{q} L_{x}^{r}(\mathbf{R} \times \mathbf{R})} \lesssim\left\|\phi_{0}\right\|_{L^{2}(\mathbf{R})}+\|F\|_{L_{t}^{\tilde{q}^{\prime}} L_{x}^{\tilde{r}^{\prime}}(\mathbf{R} \times \mathbf{R})} .
$$

In order to derive the decay estimates (1.6), it follows from Stone's formula that

$$
e^{-i t H} P_{a c}(H) f=\frac{2}{\pi i} \int_{0}^{\infty} e^{-i t \mu^{4}} \mu^{3}\left[R_{V}^{+}\left(\mu^{4}\right)-R_{V}^{-}\left(\mu^{4}\right)\right] f(x) d \mu .
$$

Hence we need to study the detailed zero expansions of the resolvent $R_{V}(z)=(H-z)^{-1}$ by the perturbations of the free resolvent $R_{0}(z)$, for which the following identity holds:

$$
R_{0}(z):=\left(\Delta^{2}-z\right)^{-1}=\frac{1}{2 z^{\frac{1}{2}}}\left(R\left(-\Delta ; z^{\frac{1}{2}}\right)-R\left(-\Delta ;-z^{\frac{1}{2}}\right)\right), \quad z \in \mathbf{C} \backslash[0, \infty) .
$$

Here the resolvent $R\left(-\Delta ; z^{\frac{1}{2}}\right):=\left(-\Delta-z^{\frac{1}{2}}\right)^{-1}$. For $\lambda \in \mathbf{R}^{+}$, we define the limiting resolvent operators by

$$
\begin{aligned}
& R_{0}^{ \pm}(\lambda):=R_{0}^{ \pm}(\lambda \pm i 0)=\lim _{\epsilon \rightarrow 0^{+}}\left(\Delta^{2}-(\lambda \pm i \epsilon)\right)^{-1}, \\
& R_{V}^{ \pm}(\lambda):=R_{V}^{ \pm}(\lambda \pm i 0)=\lim _{\epsilon \rightarrow 0^{+}}(H-(\lambda \pm i \epsilon))^{-1},
\end{aligned}
$$

By using the representation (1.14) for $R_{0}(z)$ with $z=\omega^{4}$ for $\omega$ in the first quadrant of the complex plane, and taking limits as $\omega \rightarrow \mu$ and $\omega \rightarrow i \mu$, we obtain

$$
R_{0}^{ \pm}\left(\mu^{4}\right)=\frac{1}{2 \mu^{2}}\left(R^{ \pm}\left(-\Delta ; \mu^{2}\right)-R\left(-\Delta ;-\mu^{2}\right)\right), \quad \mu>0 .
$$

It is well-known that by the limiting absorption principle ( see e.g. Agm75), $R^{ \pm}\left(-\Delta ; \mu^{2}\right)$ are well-defined as the bounded operators of $B\left(L_{s}^{2}, L_{-s}^{2}\right)$ for any $s>1 / 2$, and therefore $R_{0}^{ \pm}\left(\mu^{4}\right)$ are also well-defined between these weighted spaces. This limiting absorption property can be extended to $R_{V}^{ \pm}\left(\mu^{4}\right)$ for $\mu>0$ for certain decaying and bounded potentials ( see e.g. [FSY18] ). 
The main challenge is to derive the asymptotic expansions of $R_{V}^{ \pm}\left(\mu^{4}\right)$ as $\mu$ near zero. Recall that the Green's kernel of the free Laplace resolvent in dimension one( see [ES04, JN01] for example)

$$
R^{ \pm}\left(-\Delta ; \mu^{2}\right)(x, y)=\frac{ \pm i}{2 \mu} e^{ \pm i \mu|x-y|}
$$

Therefore, by (1.17), we have for $\mu>0$,

$$
R_{0}^{ \pm}\left(\mu^{4}\right)(x, y)=\frac{1}{4 \mu^{3}}\left( \pm i e^{ \pm i \mu|x-y|}-e^{-\mu|x-y|}\right) .
$$

Denote $M^{ \pm}(\mu)=U+v R_{0}^{ \pm}\left(\mu^{4}\right) v, v(x)=|V(x)|^{1 / 2}$ and $U=\operatorname{sgn}(V(x))$, by the following symmetric identity:

$$
R_{V}^{ \pm}\left(\mu^{4}\right)=R_{0}^{ \pm}\left(\mu^{4}\right)-R_{0}^{ \pm}\left(\mu^{4}\right) v\left(M^{ \pm}(\mu)\right)^{-1} v R_{0}^{ \pm}\left(\mu^{4}\right)
$$

It suffices to establish the asymptotic expansions of $\left(M^{ \pm}(\mu)\right)^{-1}$ with the presence of zero resonance. For this end, let's introduce some spaces to derive the asymptotic expansions of $\left(M^{ \pm}(\mu)\right)^{-1}$.

Let $T_{0}:=U+v G_{0} v$ with $G_{0}=\left(\Delta^{2}\right)^{-1}$ and $P=\|V\|_{L^{1}(\mathbf{R})}^{-1} v\langle v, \cdot\rangle$ be the orthogonal projection onto the span of $v$, i.e. $P L^{2}(\mathbf{R})=\operatorname{span}\{v\}$.

Definition 1.5. Let $Q=I-P$. Then we define that

(i) $S_{0} L^{2}(\mathbf{R})=\left\{f \in L^{2} \mid\left\langle f, x^{k} v\right\rangle=0, k=0,1\right\}$.

(ii) $S_{1} L^{2}(\mathbf{R})=\left\{f \in L^{2} \mid\left\langle f, x^{k} v\right\rangle=0, k=0,1\right.$, and $\left.S_{0} T_{0} f=0\right\}$.

(iii) $S_{2} L^{2}(\mathbf{R})=\left\{f \in L^{2} \mid\left\langle f, x^{k} v\right\rangle=0, k=0,1,2\right.$, and $\left.Q T_{0} f=0\right\}$.

(iv) $S_{3} L^{2}(\mathbf{R})=\left\{f \in L^{2} \mid\left\langle f, x^{k} v\right\rangle=0, k=0,1,2,3\right.$, and $\left.T_{0} f=0\right\}$.

Remark 1.6. (i) The projection spaces clearly satisfy the following equivalent relationships:

$$
\begin{aligned}
& f \in S_{0} L^{2}(\mathbf{R}) \Longleftrightarrow f \in \operatorname{span}\{v, x v\}^{\perp} ; \\
& f \in S_{1} L^{2}(\mathbf{R}) \Longleftrightarrow f \in \operatorname{span}\{v, x v\}^{\perp} \text { and } T_{0} f \in \operatorname{span}\{v, x v\} ; \\
& f \in S_{2} L^{2}(\mathbf{R}) \Longleftrightarrow f \in \operatorname{span}\left\{v, x v, x^{2} v\right\}^{\perp} \text { and } T_{0} f \in \operatorname{span}\{v\} ; \\
& f \in S_{3} L^{2}(\mathbf{R}) \Longleftrightarrow f \in \operatorname{span}\left\{v, x v, x^{2} v, x^{3} v\right\}^{\perp} \text { and } T_{0} f=0 .
\end{aligned}
$$

Hence, $S_{0} L^{2}(\mathbf{R}) \supseteq S_{1} L^{2}(\mathbf{R}) \supseteq S_{2} L^{2}(\mathbf{R}) \supseteq S_{3} L^{2}(\mathbf{R})$.

(ii) Denote by $S_{j}(j=0,1,2,3)$ the orthogonal projections onto the related to projection spaces $S_{j} L^{2}(\mathbf{R})$, then their orthogonal relationships can be listed in the following Table $\mathbf{1}$.

(iii) From Lemma 3.5 later, we will further prove that $S_{3} L^{2}(\mathbf{R})=\{0\}$, which is important in the proof of Theorem 1.8 (iii), see Section 3 below.

These projection spaces in Definition 1.5 will be used to characterize the zero energy resonance types of $H$ ( see Theorem 4.1 for the proof).

Theorem 1.7. Assume that $H=\Delta^{2}+V$ with $|V(x)| \lesssim(1+|x|)^{-\beta}$ with some $\beta$ satisfying the condition (1.5). Then the following statements hold:

(i) zero is a regular point of $H$ if and only if $S_{1} L^{2}(\mathbf{R})=\{0\}$;

(ii) zero is a first kind resonance of $H$ if and only if $S_{1} L^{2}(\mathbf{R}) \neq\{0\}$ and $S_{2} L^{2}(\mathbf{R})=\{0\}$;

(iii) zero is a second kind resonance of $H$ if and only if $S_{2} L^{2}(\mathbf{R}) \neq\{0\}$. 


\begin{tabular}{|c|c|}
\hline Projections on $L^{2}(\mathbf{R})$ & Orthogonal Relationships \\
\hline$S_{0}$ & $S_{0}(v(x))=S_{0}(x v(x))=0$ \\
\hline \multirow{2}{*}{$S_{1}$} & $S_{1} T_{0} S_{0}=S_{0} T_{0} S_{1}=0$ \\
& $S_{1}(v(x))=S_{1}(x v(x))=0$ \\
\hline \multirow{2}{*}{$S_{2}$} & $S_{2} T_{0} Q=Q T_{0} S_{2}=0$ \\
& $S_{2}(v(x))=S_{2}(x v(x))=S_{2}\left(x^{2} v(x)\right)=0$ \\
\hline \multirow{2}{*}{$S_{3}$} & $S_{3} T_{0} Q=Q T_{0} S_{3}=0$ \\
& $S_{3} T_{0} P=P T_{0} S_{3}=0$ \\
& $S_{3}(v(x))=S_{3}(x v(x))=0$ \\
& $S_{3}\left(x^{2} v(x)\right)=S_{3}\left(x^{3} v(x)\right)=0$ \\
\hline
\end{tabular}

TABLE 1. Orthogonal Relationships of Projections

By the characterizations of zero resonances in Theorem 1.7 and using the orthogonal relationships in Table 1, we can derive the asymptotic expansions of $\left(M^{ \pm}(\mu)\right)^{-1}$, which are fundamental to study dispersive estimates and other problems related to $H$.

Theorem 1.8. Assume that $|V(x)| \lesssim(1+|x|)^{-\beta}$ with some $\beta>0$. Then for $0<|\mu| \ll 1$, we have the following expansions of $\left(M^{ \pm}(\mu)\right)^{-1}$ in $L^{2}(\mathbf{R})$ :

(i) If zero is a regular point of $H$ and $\beta>13$, then

$$
\left(M^{ \pm}(\mu)\right)^{-1}=S_{0} A_{01}^{0} S_{0}+\mu Q A_{11}^{0} Q+\mu^{2}\left(Q A_{21}^{0} Q+S_{0} A_{22}^{0}+A_{23}^{0} S_{0}\right)+\Gamma_{3}^{0}(\mu),
$$

where $A_{k j}^{0}$ are $\mu$-independent bounded operators in $L^{2}(\mathbf{R})$ and $\Gamma_{3}^{0}(\mu)$ is $\mu$-dependent operator which satisfies

$$
\left\|\Gamma_{3}^{0}(\mu)\right\|_{L^{2} \rightarrow L^{2}}+\mu\left\|\partial_{\mu}\left(\Gamma_{3}^{0}(\mu)\right)\right\|_{L^{2} \rightarrow L^{2}} \lesssim \mu^{3} .
$$

(ii) If zero is a first kind resonance of $H$ and $\beta>17$, then

$$
\begin{aligned}
\left(M^{ \pm}(\mu)\right)^{-1}= & \frac{S_{1} A_{-1,1}^{1} S_{1}}{\mu}+S_{0} A_{01}^{1} Q+Q A_{02}^{1} S_{0}+\mu\left(Q A_{11}^{1} Q+S_{0} A_{12}^{1}+A_{13}^{1} S_{0}\right) \\
& +\mu^{2}\left(Q A_{21}^{1}+A_{22}^{1} Q\right)+\Gamma_{3}^{1}(\mu),
\end{aligned}
$$

where $A_{k j}^{1}$ are $\mu$-independent bounded operators in $L^{2}(\mathbf{R})$ and $\Gamma_{3}^{1}(\mu)$ is $\mu$-dependent operator which satisfies

$$
\left\|\Gamma_{3}^{1}(\mu)\right\|_{L^{2} \rightarrow L^{2}}+\mu\left\|\partial_{\mu}\left(\Gamma_{3}^{1}(\mu)\right)\right\|_{L^{2} \rightarrow L^{2}} \lesssim \mu^{3} .
$$

(iii) If zero is a second kind resonance of $H$ and $\beta>25$, then

$$
\begin{aligned}
\left(M^{ \pm}(\mu)\right)^{-1}= & \frac{S_{2} A_{-3,1}^{2} S_{2}}{\mu^{3}}+\frac{S_{2} A_{-2,1}^{2} S_{0}+S_{0} A_{-2,2}^{2} S_{2}}{\mu^{2}}+\frac{S_{0} A_{-1,1}^{2} S_{0}+Q A_{-1,2}^{2} S_{2}+S_{2} A_{-1,3}^{2} Q}{\mu} \\
& +\left(Q A_{01}^{2} S_{0}+S_{0} A_{02}^{2} Q+S_{2} A_{03}^{2}+A_{04}^{2} S_{2}\right)+\mu\left(Q A_{11}^{2} Q+S_{0} A_{12}^{2}+A_{13}^{2} S_{0}\right) \\
& +\mu^{2}\left(Q A_{21}^{2}+A_{21}^{2} Q\right)+\Gamma_{3}^{2}(\mu),
\end{aligned}
$$

where $A_{k j}^{2}$ are $\mu$-independent bounded operators in $L^{2}(\mathbf{R})$ and $\Gamma_{3}^{2}(\mu)$ is $\mu$-dependent operator which satisfies

$$
\left\|\Gamma_{3}^{2}(\mu)\right\|_{L^{2} \rightarrow L^{2}}+\mu\left\|\partial_{\mu}\left(\Gamma_{3}^{2}(\mu)\right)\right\|_{L^{2} \rightarrow L^{2}} \lesssim \mu^{3} .
$$


Remark 1.9. If we assume the more decay rate of $V(x)$, then we will have more information about the error term $\Gamma_{3}^{i}(\mu)$ ( here the index $i$ respects to the zero energy type). More specifically, one actually can obtain that

$$
\Gamma_{3}^{i}(\mu)=\frac{\mu^{3}}{\tilde{a}^{ \pm}} P+\mu^{3}\left(Q A_{31}^{i}+A_{32}^{i} Q\right)+\Gamma_{4}^{i}(\mu),
$$

where $A_{31}^{i}$ are $\mu$-independent bounded operators in $L^{2}(\mathbf{R})$ and $\Gamma_{4}^{i}(\mu)$ are $\mu$-dependent operators which satisfy

$$
\left\|\Gamma_{4}^{i}(\mu)\right\|_{L^{2} \rightarrow L^{2}}+\mu\left\|\partial_{\mu}\left(\Gamma_{4}^{i}(\mu)\right)\right\|_{L^{2} \rightarrow L^{2}} \lesssim \mu^{4}
$$

Remark 1.10. In addtion, assuming the less decay rate of $V(x)$, we can also obtain some weak expansions of $\left(M^{ \pm}(\mu)\right)^{-1}$ by checking the proof process of Theorem 1.8 (see Section 3) along with Remark 3.2. Specifically, in the Regular Case, it is enough for $\beta>7$ to obtain the following expansions:

$$
\left(M^{ \pm}(\mu)\right)^{-1}=S_{0} \Gamma_{01}^{0}(\mu) S_{0}+Q \Gamma_{11}^{0}(\mu) Q+\left(Q \Gamma_{21}^{0}(\mu) Q+S_{0} \Gamma_{22}^{0}(\mu)+\Gamma_{23}^{0}(\mu) S_{0}\right)+\Gamma_{31}^{0}(\mu),
$$

where $\Gamma_{k j}^{0}(\mu)(k=0,1,2,3)$ are $\mu$-dependent operators which satisfy

$$
\left\|\Gamma_{k j}^{0}(\mu)\right\|_{L^{2} \rightarrow L^{2}}+\mu\left\|\partial_{\mu}\left(\Gamma_{k j}^{0}(\mu)\right)\right\|_{L^{2} \rightarrow L^{2}} \lesssim \mu^{k} .
$$

Furthermore, similar weak expansions of $\left(M^{ \pm}(\mu)\right)^{-1}$ can be established for the different resonance cases if $V$ satisfies the following decay rate:

$$
\beta> \begin{cases}9, & \text { zero is the first kind resonance, } \\ 13, & \text { zero is the second kind resonance. }\end{cases}
$$

According to the proof of Theorem 1.3, we remark that even if we use the above weak form expansion of $\left(M^{ \pm}(\mu)\right)^{-1}$, the $L^{1}-L^{\infty}$ decay estimates (1.6) of $e^{-i t H}$ also hold. But the expansions of Theorem 1.8 will be helpful to other problems such as the $L^{p}$ boundedness of the wave operators.

From Theorem 1.8 above, it is obvious that all types of zero energy resonances lead to the higher singularity than the regular case in the expansions of $\left(M^{ \pm}(\mu)\right)^{-1}$. On the other hand, the zero energy resonances also offer more orthogonal relationships, which in turn provide the more cancellations to balance the singularities at the cost of the decay rate of potentials, as shown in the proof of Theorem 1.3 below.

We also remark that our analysis for the proof of Theorem 1.8 is longer and more complicated processes in some ways. First, some singular terms in the expansions of the free resolvent $R_{0}^{ \pm}\left(\mu^{4}\right)$ will bring more complicated interacting terms in the expansions of $\left(M^{ \pm}(\mu)\right)^{-1}$, see Section 3 , Then these interacting terms will lead to the difficulties of the inversion processes, especially for the second kind resonance case. For instance, in the case of the second kind resonance, we need to analyze the kernel of the non-positive operator $T_{2}$ given by

$$
\begin{aligned}
T_{2} & =S_{2} v G_{2} v S_{2}-\frac{8 \cdot(6 !)}{\|V\|_{L^{1}}} S_{2} T_{0}^{2} S_{2}+\frac{5}{2} S_{2} v G_{1} v D_{0} v G_{1} v S_{2} \\
& +\frac{48 \cdot(6 !)}{\|V\|_{L^{1}}^{2}}\left(S_{2} T_{0} v G_{-1} v-\frac{\|V\|_{L^{1}}}{6} S_{2} v G_{1} v D_{0} T_{0}\right) D_{2}\left(v G_{-1} v T_{0} S_{2}-\frac{\|V\|_{L^{1}}}{6} T_{0} D_{0} v G_{1} v S_{2}\right),
\end{aligned}
$$

which is actually a sum of the four complicated operators with changing signs. We will identify the ker $T_{2}$ as the projection space $S_{3} L^{2}(\mathbf{R})$, see Lemma 3.4 for more details.

Finally, let us comment about the proof of the decay estimates in Theorem 1.3 . 
Since the derivative of $\mu$ for the free resolvent kernel $R_{0}^{ \pm}\left(\mu^{4}\right)(x, y)$ can not be dominated by $\mu^{\alpha}$ in $x, y$ uniformly, in this paper we have used Littlewood-Paley decompositions and oscillatory integrals theory to solve the uniformity problem, which seems to be general interesting technical point. Using some similar methods but with important technical modifications, Li, Soffer and Yao LSY21] have established the $L^{1}-L^{\infty}$ estimates for $e^{-i t H}$ in dimension two. We also think that these arguments used here could be extended to general higher-order elliptic operators $P(D)+V$.

The paper is organized as follows. In Section 2, we give the proof of Theorem 1.3. In Section

3 , we give the process how to obtain the asymptotic expansion of $\left(M^{ \pm}(\mu)\right)^{-1}$ case by case. In Section 4, we characterize the resonance spaces for each kind of zero resonance.

\section{Proof of Theorem 1.3}

In this section, we will give the proof of Theorem 1.3. Choose a fixed even function $\varphi \in C_{c}^{\infty}(\mathbf{R})$ such that

$$
\varphi(s)= \begin{cases}1, & \text { if }|s| \leq \frac{1}{2} \\ 0, & \text { if }|s| \geq 1\end{cases}
$$

For each $N \in \mathbf{Z}$, let $\varphi_{N}(s)=\varphi\left(2^{-N} s\right)-\varphi\left(2^{-N+1} s\right)$, then $\operatorname{supp} \varphi_{N} \subset\left[2^{N-2}, 2^{N}\right]$ and $\varphi_{N}(s)=$ $\varphi_{0}\left(2^{-N} s\right)$. Furthermore, for any $s \neq 0$ we have $\sum_{N=-\infty}^{+\infty} \varphi_{N}(s)=1$. Using functional calculus and Stone's formula, we have

$$
\begin{aligned}
e^{-i t H} P_{a c}(H) f= & \frac{2}{\pi i} \int_{0}^{\infty} e^{-i t \mu^{4}} \mu^{3}\left[R_{V}^{+}\left(\mu^{4}\right)-R_{V}^{-}\left(\mu^{4}\right)\right] f d \mu \\
= & \frac{2}{\pi i} \int_{0}^{\infty} e^{-i t \mu^{4}} \sum_{N=-\infty}^{+\infty} \varphi_{N}(\mu) \mu^{3}\left[R_{V}^{+}\left(\mu^{4}\right)-R_{V}^{-}\left(\mu^{4}\right)\right] f d \mu \\
:= & \frac{2}{\pi i} \int_{0}^{\infty} e^{-i t \mu^{4}} \chi(\mu) \mu^{3}\left[R_{V}^{+}\left(\mu^{4}\right)-R_{V}^{-}\left(\mu^{4}\right)\right] f d \mu \\
& +\frac{2}{\pi i} \int_{0}^{\infty} e^{-i t \mu^{4}} \widetilde{\chi}(\mu) \mu^{3}\left[R_{V}^{+}\left(\mu^{4}\right)-R_{V}^{-}\left(\mu^{4}\right)\right] f d \mu,
\end{aligned}
$$

where $\chi(\mu)=\sum_{N=-\infty}^{N_{0}} \varphi_{N}(\mu)$ and $\tilde{\chi}(\mu)=\sum_{N=N_{0}+1}^{+\infty} \varphi_{N}(\mu)$ for some $N_{0}<0$ ( fixed later but depending on specific zero energy types). Hence we reduce the proof of Theorem 1.3 into the estimates of the following two parts: low and high energy parts.

Theorem 2.1. (the low energy dispersive estimate) Let $|V(x)| \lesssim(1+|x|)^{-\beta}$ with some $\beta>0$ depending on the following zero energy types:

$$
\beta>\left\{\begin{array}{l}
13, \quad \text { zero is the regular point, } \\
17, \quad \text { zero is the first kind resonance, } \\
25, \text { zero is the second kind resonance. }
\end{array}\right.
$$

Assume that $H=\Delta^{2}+V$ has no positive embedded eigenvalue and $P_{a c}(H)$ denotes the projection onto the absolutely continuous spectrum space of $H$, then the following estimate holds:

$$
\left\|e^{-i t H} P_{a c}(H) \chi(H)\right\|_{L^{1}(\mathbf{R}) \rightarrow L^{\infty}(\mathbf{R})} \lesssim|t|^{-\frac{1}{4}} .
$$

Moreover, for any $0 \leq \alpha<1$, we have the following estimate:

$$
\left\|H^{\frac{\alpha}{4}} e^{-i t H} P_{a c}(H) \chi(H)\right\|_{L^{1}(\mathbf{R}) \rightarrow L^{\infty}(\mathbf{R})} \lesssim|t|^{-\frac{1+\alpha}{4}} .
$$




\section{Theorem 2.2. (the high energy dispersive estimate)}

Let $|V(x)| \lesssim(1+|x|)^{-2-}$. Assume that $H=\Delta^{2}+V$ has no positive embedded eigenvalue and $P_{a c}(H)$ denotes the projection onto the absolutely continuous spectrum space of $H$, then we have

$$
\left\|e^{-i t H} P_{a c}(H) \widetilde{\chi}(H)\right\|_{L^{1}(\mathbf{R}) \rightarrow L^{\infty}(\mathbf{R})} \lesssim|t|^{-\frac{1}{4}} .
$$

Moreover, for any $0<\alpha<1$, we have the following estimate:

$$
\left\|H^{\frac{\alpha}{4}} e^{-i t H} P_{a c}(H) \widetilde{\chi}(H)\right\|_{L^{1}(\mathbf{R}) \rightarrow L^{\infty}(\mathbf{R})} \lesssim|t|^{-\frac{1+\alpha}{4}} .
$$

The proofs of the low and high energy parts will be given in the following subsections. For the end, let us first prove the decay estimates of the free group $e^{-i t \Delta^{2}}$ in dimension one by the resolvent method, which display some basic technics how to prove the potential case.

2.1. The estimates for the free case. First, we give a useful lemma to estimates a kind of oscillatory integral appeared repeatedly in this paper.

Lemma 2.3. Let $m \geq 1, \Psi(z)$ be a nonnegative real valued function of $\mathbf{R}^{m}$ and $\Phi(s ; z)$ be a function on $\mathbf{R} \times \mathbf{R}^{m}$, which is smooth for the first variable $s$. Assume that for any $s \in \operatorname{supp} \varphi_{0}(s)$ (here $\varphi_{0}(s)=\varphi(s)-\varphi\left(\frac{1}{2} s\right)$, see (2.1)) and any $N \in \mathbf{Z}, \Phi(s ; z)$ satisfies the following uniform bounded condition:

then we have

$$
\left|\partial_{s}^{k} \Phi\left(2^{N} s ; z\right)\right| \lesssim 1, \quad k=0,1
$$

$$
\sup _{z \in \mathbf{R}^{m}}\left|\int_{0}^{\infty} e^{-i t 2^{4 N} s^{4}} \varphi_{0}(s) e^{ \pm i 2^{N} s \Psi(z)} \Phi\left(2^{N} s ; z\right) d s\right| \lesssim\left(1+|t| \cdot 2^{4 N}\right)^{-\frac{1}{2}} .
$$

Proof. Denote

$$
K_{N}^{ \pm}(t, z):=\int_{0}^{\infty} e^{-i t 2^{4 N} s^{4}} \varphi_{0}(s) e^{ \pm i 2^{N} s \Psi(z)} \Phi\left(2^{N} s ; z\right) d s .
$$

Since for any $s \in \operatorname{supp} \varphi_{0}(s) \subset\left[\frac{1}{4}, 1\right],\left|\Phi\left(2^{N} s ; z\right)\right| \lesssim 1$, then we have

$$
\left|K_{N}^{ \pm}(t, z)\right| \lesssim \int_{\frac{1}{4}}^{1}\left|\varphi_{0}(s)\right| d s \lesssim 1 .
$$

Case 1: $2^{N} \Psi(z) \leq 1$. Using integrate by parts once, we have

$$
\begin{aligned}
K_{N}^{ \pm}(t, z)=\left.\frac{e^{-i t 2^{4 N} s^{4}}\left(s^{-3} \varphi_{0}(s) e^{ \pm i 2^{N} s \Psi(z)} \Phi\left(2^{N} s ; z\right)\right)}{-4 i t \cdot 2^{4 N}}\right|_{0} ^{\infty} \\
\quad+\frac{1}{4 i t \cdot 2^{4 N}} \int_{0}^{\infty} e^{-i t 2^{4 N} s^{4}} \partial_{s}\left(s^{-3} \varphi_{0}(s) e^{ \pm i 2^{N} s \Psi(z)} \Phi\left(2^{N} s ; z\right)\right) d s .
\end{aligned}
$$

Noting that for any $s \in \operatorname{supp} \varphi_{0}(s)$ and $k=0,1$,

$$
\left|\partial_{s}^{k}\left(e^{ \pm i 2^{N} s \Psi(z)}\right)\right| \lesssim 1, \quad \mid \partial_{s}^{k}\left(\Phi\left(2^{N} s ; z\right) \mid \lesssim 1\right.
$$

thus we can obtain that

$$
\left|K_{N}^{ \pm}(t, z)\right| \lesssim\left(|t| \cdot 2^{4 N}\right)^{-1}
$$

Combining (2.8) with (2.9), we obtain that

$$
\sup _{z \in \mathbf{R}^{m}}\left|K_{N}^{ \pm}(t, z)\right| \lesssim\left(1+|t| \cdot 2^{4 N}\right)^{-\frac{1}{2}}
$$

Case 2: $2^{N} \Psi(z) \geq 1$. Set $r=2^{N} \Psi(z)$. In this case, we rewrite $K_{N}^{ \pm}(t, z)$ as follows:

$$
K_{N}^{ \pm}(t, z)=\int_{0}^{\infty} e^{-i t 2^{4 N} s^{4} \pm i s r} \varphi_{0}(s) \Phi\left(2^{N} s ; z\right) d s
$$


Assume that $t>0$, we consider $K_{N}^{-}(t, z)$ and $K_{N}^{+}(z)$ case by case.

Firstly, we study $K_{N}^{-}(t, z)$. Let $u_{-}(s, r)=t \cdot 2^{4 N} s^{4}+s r$, then we have

$$
K_{N}^{-}(t, z)=\int_{0}^{\infty} e^{-i u_{-}(s, r)} \varphi_{0}(s) \Phi\left(2^{N} s ; z\right) d s .
$$

It is easy to know that

$$
\left|\partial_{s} u_{-}(s, r)\right|=\left|4 t \cdot 2^{4 N} s^{3}+r\right| \gtrsim\left(1+|t| \cdot 2^{4 N}\right) .
$$

Thus $u_{-}(s, r)$ does not exist critical point, by (2.8) and integrating by parts once, we have

$$
\left|K_{N}^{-}(t, z)\right| \lesssim\left(1+|t| \cdot 2^{4 N}\right)^{-\frac{1}{2}}
$$

Next to consider $K_{N}^{+}(t, z)$. Let $u_{+}(s, r)=t \cdot 2^{4 N} s^{4}-s r$, then we have

$$
K_{N}^{+}(t, z)=\int_{0}^{\infty} e^{-i u_{+}(s, r)} \varphi_{0}(s) \Phi\left(2^{N} s ; z\right) d s .
$$

It is obvious that

$$
\partial_{s} u_{+}(s, r)=4 t \cdot 2^{4 N} s^{3}-r
$$

has a critical point $s_{0}$ such $r=4 t \cdot 2^{4 N} s_{0}^{3}$.

If $r \geq 8 t \cdot 2^{4 N}$ or $r \leq \frac{1}{32} t \cdot 2^{4 N}$, then the such critical point $s_{0}$ does not exist in the range $[1 / 4,1]$, so by (2.8) and using integration by parts once, we have

$$
\left|K_{N}^{+}(t, z)\right| \lesssim\left(1+|t| \cdot 2^{4 N}\right)^{-\frac{1}{2}}
$$

If $\frac{1}{32} t \cdot 2^{4 N} \leq r \leq 8 t \cdot 2^{4 N}$, then the critical point exists. But since $\left|\partial_{s}^{2} u_{+}(s, r)\right|=\left|12 t \cdot 2^{4 N} s^{2}\right| \gtrsim$ $|t| \cdot 2^{4 N}$, thus by Van der Corput lemma ( see e.g. [Ste93]), we have

$$
\left|K_{N}^{+}(t, z)\right| \lesssim\left(1+|t| \cdot 2^{4 N}\right)^{-\frac{1}{2}}
$$

Hence, by summing all cases above we have that

$$
\sup _{z \in \mathbf{R}^{m}}\left|K_{N}^{ \pm}(t, z)\right| \lesssim\left(1+|t| \cdot 2^{4 N}\right)^{-\frac{1}{2}}
$$

which concludes the proof of this lemma.

Using Lemma 2.3, we can give the following decay estimates of $e^{-i t \Delta^{2}}$ in one dimensional case by the free resolvent $R_{0}^{ \pm}\left(\mu^{4}\right)$. Note that we can write down

$$
e^{-i t \Delta^{2}} f(x)=\int_{\mathbf{R}}\left(\frac{2}{\pi i} \int_{0}^{\infty} e^{-i t \mu^{4}} \mu^{3}\left[R_{0}^{+}\left(\mu^{4}\right)-R_{0}^{-}\left(\mu^{4}\right)\right](x, y) d \mu\right) f(y) d y
$$

where

$$
R_{0}^{ \pm}\left(\mu^{4}\right)(x, y)=\frac{1}{4 \mu^{3}}\left( \pm i e^{ \pm i \mu|x-y|}-e^{-\mu|x-y|}\right)=\frac{e^{ \pm i \mu|x-y|}}{4 \mu^{3}}\left( \pm i-e^{-\mu|x-y| \mp i \mu|x-y|}\right) .
$$

Proposition 2.4. For any $N \in \mathbf{Z}$, we have

$$
\sup _{x, y \in \mathbf{R}}\left|\int_{0}^{\infty} e^{-i t \mu^{4}} \mu^{3} \varphi_{N}(\mu) R_{0}^{ \pm}\left(\mu^{4}\right)(x, y) d \mu\right| \lesssim 2^{N}\left(1+|t| \cdot 2^{4 N}\right)^{-\frac{1}{2}} .
$$

Furthermore, we have

$$
\sup _{x, y \in \mathbf{R}}\left|\int_{0}^{\infty} e^{-i t \mu^{4}} \mu^{3} R_{0}^{ \pm}\left(\mu^{4}\right)(x, y) d \mu\right| \lesssim|t|^{-\frac{1}{4}} .
$$

As a consequence, it follows that

$$
\left\|e^{-i t \Delta^{2}}\right\|_{L^{1}(\mathbf{R}) \rightarrow L^{\infty}(\mathbf{R})} \lesssim|t|^{-\frac{1}{4}}, \quad t \neq 0 .
$$


Proof. Since $\varphi_{N}(\mu)=\varphi_{0}\left(2^{-N} \mu\right)$, thus set

$$
K_{0, N}^{ \pm}(t ; x, y):=\int_{0}^{\infty} e^{-i t \mu^{4}} \mu^{3} \varphi_{0}\left(2^{-N} \mu\right)\left[R_{0}^{ \pm}\left(\mu^{4}\right)\right](x, y) d \mu .
$$

Let $\mu=2^{N} s$, then we have

$$
K_{0, N}^{ \pm}(t ; x, y)=2^{N} \int_{0}^{\infty} e^{-i t 2^{4 N} s^{4}} \varphi_{0}(s) e^{ \pm i 2^{N} s|x-y|}\left( \pm i-e^{-2^{N} s|x-y| \mp i 2^{N} s|x-y|}\right) d s .
$$

Let $z=(x, y)$, and

$$
\Psi(z)=\Psi(x, y)=|x-y|, \quad \Phi^{ \pm}(s ; z)=\Phi^{ \pm}(s ; x, y)= \pm i-e^{-s|x-y| \mp i s|x-y|} .
$$

It is obvious that for any $s \in \operatorname{supp} \varphi_{0}(s)$ and $k=0,1,\left|\partial_{s}^{k}\left(\Phi^{ \pm}\left(2^{N} s ; x, y\right)\right)\right| \lesssim 1$, then by Lemma 2.3, we obtain that

$$
\sup _{x, y \in \mathbf{R}}\left|K_{0, N}^{ \pm}(t ; x, y)\right| \lesssim 2^{N}\left(1+|t| \cdot 2^{4 N}\right)^{-\frac{1}{2}}
$$

Denote

$$
K_{0}^{ \pm}(t ; x, y)=\int_{0}^{\infty} e^{-i t \mu^{4}} \mu^{3} R_{0}^{ \pm}\left(\mu^{4}\right)(x, y) d \mu
$$

Thus

$$
\begin{aligned}
K_{0}^{ \pm}(t ; x, y) & =\sum_{N=-\infty}^{\infty} \int_{0}^{\infty} e^{-i t \mu^{4}} \mu^{3} \varphi_{N}(\mu)\left[R_{0}^{ \pm}\left(\mu^{4}\right)\right](x, y) d \mu \\
& =\sum_{N=-\infty}^{+\infty} K_{0, N}^{ \pm}(t ; x, y) .
\end{aligned}
$$

It is obvious that there exists $N^{\prime} \in \mathbf{Z}$ such that $|t| \cdot 2^{4 N^{\prime}} \sim 1$, so we have

$$
\begin{aligned}
\left|K_{0}^{ \pm}(t ; x, y)\right| & \lesssim \sum_{N=-\infty}^{N^{\prime}}\left|K_{0, N}^{ \pm}(t ; x, y)\right|+\sum_{N=N^{\prime}+1}^{+\infty}\left|K_{0, N}^{ \pm}(t ; x, y)\right| \\
& \lesssim \sum_{N=-\infty}^{N^{\prime}} 2^{N}\left(1+|t| \cdot 2^{4 N}\right)^{-\frac{1}{2}}+\sum_{N=N^{\prime}+1}^{+\infty} 2^{N}\left(1+|t| \cdot 2^{4 N}\right)^{-\frac{1}{2}} \\
& \lesssim \sum_{N=-\infty}^{N^{\prime}} 2^{N}+|t|^{-\frac{1}{2}} \sum_{N=N^{\prime}+1}^{+\infty} 2^{-N} \\
& \lesssim|t|^{-\frac{1}{4}},
\end{aligned}
$$

which immediately gives the (2.12).

2.2. Low Energy Dispersive Estimate with $\alpha=0$. In this subsection, we show the low energy dispersive estimate (2.3) case by case for zero is a regular point or zero energy resonance.

The following lemma play an important role to make use of cancellations from projections operator $S_{j}$ in the zero asymptotical expansions of resolvent $R_{V}(z)$ ( see Theorem 1.8 above ), and will be used repeatedly to obtain low energy dispersive estimates for all cases.

Lemma 2.5. Let $\mu>0$ and $\operatorname{sgn}(x)$ be the sign function of $x$ on $\mathbf{R}$. Then

(i) If $F(p) \in C^{1}(\mathbf{R})$, then for any $x, y \in \mathbf{R}$, we have

$$
F(\mu|x-y|)=F(\mu|x|)-\mu y \int_{0}^{1} \operatorname{sgn}(x-\theta y) F^{\prime}(\mu|x-\theta y|) d \theta .
$$


(ii) If $F(p) \in C^{2}(\mathbf{R})$ and $F^{\prime}(0)=0$, then for any $x, y \in \mathbf{R}$, we have

$$
F(\mu|x-y|)=F(\mu|x|)-\mu y \operatorname{sgn}(x) F^{\prime}(\mu|x|)+\mu^{2} y^{2} \int_{0}^{1}(1-\theta) F^{\prime \prime}(\mu|x-\theta y|) d \theta .
$$

(iii) If $F(p) \in C^{3}(\mathbf{R})$ and $F^{\prime}(0)=F^{\prime \prime}(0)=0$, then for any $x, y \in \mathbf{R}$, we have

$$
\begin{aligned}
F(\mu|x-y|)= & F(\mu|x|)-\mu y \operatorname{sgn}(x) F^{\prime}(\mu|x|)+\frac{\mu^{2} y^{2}}{2 !} F^{\prime \prime}(\mu|x|) \\
& -\frac{\mu^{3} y^{3}}{2 !} \int_{0}^{1}(1-\theta)^{2}(\operatorname{sgn}(x-\theta y))^{3} F^{(3)}(\mu|x-\theta y|) d \theta .
\end{aligned}
$$

Proof. Denote

$$
G_{\varepsilon}(y)=F\left(\mu \sqrt{\varepsilon^{2}+(x-y)^{2}}\right), \quad \varepsilon \neq 0 .
$$

If $F(p) \in C^{n+1}(\mathbf{R})$, then $G_{\varepsilon}(y) \in C^{n+1}(\mathbf{R})$ for $\varepsilon \neq 0$. By Taylor expansions, we have

$$
G_{\varepsilon}(y)=\sum_{k=0}^{n} \frac{G_{\varepsilon}^{(k)}(0)}{k !} y^{k}+\frac{y^{n+1}}{n !} \int_{0}^{1}(1-\theta)^{n} G_{\varepsilon}^{(n+1)}(\theta y) d \theta .
$$

(i) Since $F(p) \in C^{1}(\mathbf{R})$, then $\lim _{\varepsilon \rightarrow 0} G_{\varepsilon}(y)=\lim _{\varepsilon \rightarrow 0} F\left(\mu \sqrt{\varepsilon^{2}+(x-y)^{2}}\right)=F(\mu|x-y|)$. Note that for $\varepsilon \neq 0$,

$$
G_{\varepsilon}^{\prime}(y)=\frac{-\mu(x-y)}{\sqrt{\left(\varepsilon^{2}+(x-y)^{2}\right)}} F^{\prime}\left(\mu \sqrt{\varepsilon^{2}+(x-y)^{2}}\right),
$$

then for each $0 \leq \theta \leq 1$ and $x, y \in \mathbf{R}$,

$$
\lim _{\varepsilon \rightarrow 0} G_{\varepsilon}^{\prime}(\theta y)=-\mu \operatorname{sgn}(x-\theta y) F^{\prime}(\mu|x-\theta y|) .
$$

Since there exists a constant $C=C(\mu, x, y)>0$ such that $\left|G_{\varepsilon}^{\prime}(\theta y)\right| \leq C$ for all $0 \leq \theta \leq 1$ and $0<\varepsilon \leq 1$, then by taking $\varepsilon \downarrow 0$ in the two sides of (2.18) with $n=0$ and Lebesgue's dominated convergence theorem, it immediately follows that

$$
F(\mu|x-y|)=F(\mu|x|)-\mu y \int_{0}^{1} \operatorname{sgn}(x-\theta y) F^{\prime}(\mu|x-\theta y|) d \theta .
$$

(ii) If $F(p) \in C^{2}(\mathbf{R})$, then for $\varepsilon \neq 0$,

$$
\begin{aligned}
G_{\varepsilon}^{\prime \prime}(y)= & \mu^{2}\left(\frac{x-y}{\sqrt{\varepsilon^{2}+(x-y)^{2}}}\right)^{2} F^{\prime \prime}\left(\mu \sqrt{\varepsilon^{2}+(x-y)^{2}}\right) \\
& +\mu \frac{\varepsilon^{2}}{\left(\sqrt{\varepsilon^{2}+(x-y)^{2}}\right)^{2}} \cdot \frac{F^{\prime}\left(\mu \sqrt{\varepsilon^{2}+(x-y)^{2}}\right)}{\sqrt{\varepsilon^{2}+(x-y)^{2}}} .
\end{aligned}
$$

Since $F^{\prime}(0)=0$, thus we can obtain that

$$
\lim _{\varepsilon \rightarrow 0} G_{\varepsilon}^{\prime \prime}(y)=\mu^{2} F^{\prime \prime}(\mu|x-y|), x, y \in \mathbf{R} .
$$

Then by using (2.18) with $n=1$, (2.19), (2.20) and Lebesgue's dominated convergence theorem, we can conclude that

$$
\begin{aligned}
F(\mu|x-y|) & =\sum_{k=0}^{1} \frac{\lim _{\varepsilon \rightarrow 0} G_{\varepsilon}^{(k)}(0)}{k !} y^{k}+y^{2} \cdot \lim _{\varepsilon \rightarrow 0} \int_{0}^{1}(1-\theta) G_{\varepsilon}^{\prime \prime}(\theta y) d \theta . \\
& =F(\mu|x|)-\mu y \operatorname{sgn}(x) F^{\prime}(\mu|x|)+\mu^{2} y^{2} \int_{0}^{1}(1-\theta) F^{\prime \prime}(\mu|x-\theta y|) d \theta .
\end{aligned}
$$


(iii) If $F(p) \in C^{3}(\mathbf{R})$, then for $\varepsilon \neq 0$,

$$
\begin{aligned}
G_{\varepsilon}^{(3)}(y)= & -\mu^{3}\left(\frac{x-y}{\sqrt{\varepsilon^{2}+(x-y)^{2}}}\right)^{3} F^{(3)}\left(\mu \sqrt{\varepsilon^{2}+(x-y)^{2}}\right) \\
& -3 \mu^{2} \frac{(x-y) \varepsilon^{2}}{\left(\sqrt{\varepsilon^{2}+(x-y)^{2}}\right)^{3}} \cdot \frac{F^{\prime \prime}\left(\mu \sqrt{\varepsilon^{2}+(x-y)^{2}}\right)}{\sqrt{\varepsilon^{2}+(x-y)^{2}}} \\
& +3 \mu \frac{(x-y) \varepsilon^{2}}{\left(\sqrt{\varepsilon^{2}+(x-y)^{2}}\right)^{3}} \cdot \frac{F^{\prime}\left(\mu \sqrt{\varepsilon^{2}+(x-y)^{2}}\right)}{\left(\sqrt{\varepsilon^{2}+(x-y)^{2}}\right)^{2}} .
\end{aligned}
$$

Then for each $x, y \in \mathbf{R}$, we have

$$
\lim _{\varepsilon \rightarrow 0} G_{\varepsilon}^{(3)}(y)=-\mu^{3}(\operatorname{sgn}(x-y))^{3} F^{(3)}(\mu|x-y|) .
$$

Since $F^{\prime}(0)=F^{\prime \prime}(0)=0$, so by using the expansion (2.18) with $n=2$, the equalities (2.19) -(2.21) and Lebesgue's dominated convergence theorem, we can similarly obtain that

$$
\begin{aligned}
F(\mu|x-y|)= & \sum_{k=0}^{2} \frac{\lim _{\varepsilon \rightarrow 0} G_{\varepsilon}^{(k)}(0)}{k !} y^{k}+\frac{y^{3}}{2 !} \cdot \lim _{\varepsilon \rightarrow 0} \int_{0}^{1}(1-\theta)^{2} G_{\varepsilon}^{(3)}(\theta y) d \theta \\
= & F(\mu|x|)-\mu y \operatorname{sgn}(x) F^{\prime}(\mu|x|)+\frac{\mu^{2} y^{2}}{2 !} F^{\prime \prime}(\mu|x|) \\
& \quad-\frac{\mu^{3} y^{3}}{2 !} \int_{0}^{1}(1-\theta)^{2}(\operatorname{sgn}(x-\theta y))^{3} F^{(3)}(\mu|x-\theta y|) d \theta .
\end{aligned}
$$

Lemma 2.6. For any $0 \leq \alpha<1$, we have

$$
\sum_{N=-\infty}^{+\infty} 2^{(1+\alpha) N}\left(1+|t| \cdot 2^{4 N}\right)^{-\frac{1}{2}} \lesssim|t|^{-\frac{1+\alpha}{4}} .
$$

Proof. Since for any $t \neq 0$, there exists a $N_{0}^{\prime} \in \mathbf{Z}$ such that $|t| \cdot 2^{4 N_{0}^{\prime}} \sim 1$, thus we have

$$
\begin{aligned}
\sum_{N=-\infty}^{+\infty} 2^{(1+\alpha) N}\left(1+|t| \cdot 2^{4 N}\right)^{-\frac{1}{2}} & =\sum_{N=-\infty}^{N_{0}^{\prime}} 2^{(1+\alpha) N}\left(1+|t| \cdot 2^{4 N}\right)^{-\frac{1}{2}}+\sum_{N=N_{0}^{\prime}+1}^{+\infty} 2^{(1+\alpha) N}\left(1+|t| \cdot 2^{4 N}\right)^{-\frac{1}{2}} \\
& \lesssim \sum_{N=-\infty}^{N_{0}^{\prime}} 2^{(1+\alpha) N}+|t|^{-\frac{1}{2}} \sum_{N=N_{0}^{\prime}+1}^{+\infty} 2^{(\alpha-1) N} \\
& \lesssim|t|^{-\frac{1+\alpha}{4}} .
\end{aligned}
$$

2.2.1. Regular Case. In order to establish the lower energy estimate (2.3) with $\alpha=0$ in regular case, recall that by Stone's formula ( also see the equality (2.2))

$$
\begin{aligned}
e^{-i t H} P_{a c}(H) \chi(H) f & =\frac{2}{\pi i} \int_{0}^{\infty} e^{-i t \mu^{4}} \chi(\mu) \mu^{3}\left[R_{V}^{+}\left(\mu^{4}\right)-R_{V}^{-}\left(\mu^{4}\right)\right] f d \mu \\
& =\sum_{N=-\infty}^{N_{0}} \sum_{ \pm} \frac{ \pm 1}{2 \pi i} \int_{0}^{\infty} e^{-i t \mu^{4}} \varphi_{N}(\mu) \mu^{3} R_{V}^{ \pm}\left(\mu^{4}\right) f d \mu .
\end{aligned}
$$


When zero is a regular point of the spectrum of $H$, by (1.20) and (1.21), we have

$$
\begin{aligned}
R_{V}^{ \pm}\left(\mu^{4}\right)= & R_{0}^{ \pm}\left(\mu^{4}\right)-R_{0}^{ \pm}\left(\mu^{4}\right) v\left(S_{0} A_{01}^{0} S_{0}\right) v R_{0}^{ \pm}\left(\mu^{4}\right)-R_{0}^{ \pm}\left(\mu^{4}\right) v\left(\mu Q A_{11}^{0} Q\right) v R_{0}^{ \pm}\left(\mu^{4}\right) \\
& -R_{0}^{ \pm}\left(\mu^{4}\right) v\left(\mu^{2} Q A_{21}^{0} Q\right) v R_{0}^{ \pm}\left(\mu^{4}\right)-R_{0}^{ \pm}\left(\mu^{4}\right) v\left(\mu^{2} S_{0} A_{22}^{0}+\mu^{2} A_{23}^{0} S_{0}\right) v R_{0}^{ \pm}\left(\mu^{4}\right) \\
& -R_{0}^{ \pm}\left(\mu^{4}\right) v \Gamma_{3}^{0}(\mu) v R_{0}^{ \pm}\left(\mu^{4}\right) .
\end{aligned}
$$

If we substitute the (2.25) into the (2.23), and combine Proposition 2.4 and Lemma 2.6, then it suffices to show the following Propositions 2.7, 2.8, 2.9 and 2.10 corresponding to each different terms of the resolvent formula (2.25) above.

Proposition 2.7. For any $N \in \mathbf{Z}_{-}$and $N \leq N_{0}$, we have

$$
\sup _{x, y \in \mathbf{R}}\left|\int_{0}^{\infty} e^{-i t \mu^{4}} \mu^{3} \varphi_{N}(\mu)\left[R_{0}^{ \pm}\left(\mu^{4}\right) v\left(S_{0} A_{01}^{0} S_{0}\right) v R_{0}^{ \pm}\left(\mu^{4}\right)\right](x, y) d \mu\right| \lesssim 2^{2 N}\left(1+|t| \cdot 2^{4 N}\right)^{-\frac{1}{2}} .
$$

Proof. Since $\varphi_{N}(\mu)=\varphi_{0}\left(2^{-N} \mu\right)$, thus

$$
\begin{aligned}
K_{1, N}^{0, \pm}(t ; x, y) & :=\int_{0}^{\infty} e^{-i t \mu^{4}} \mu^{3} \varphi_{N}(\mu)\left[R_{0}^{ \pm}\left(\mu^{4}\right) v\left(S_{0} A_{01}^{0} S_{0}\right) v R_{0}^{ \pm}\left(\mu^{4}\right)\right](x, y) d \mu \\
& =\int_{0}^{\infty} e^{-i t \mu^{4}} \mu^{3} \varphi_{0}\left(2^{-N} \mu\right)\left[R_{0}^{ \pm}\left(\mu^{4}\right) v\left(S_{0} A_{01}^{0} S_{0}\right) v R_{0}^{ \pm}\left(\mu^{4}\right)\right](x, y) d \mu .
\end{aligned}
$$

Indeed, since

$$
R_{0}^{ \pm}(x, y)=\frac{ \pm i e^{ \pm i \mu|x-y|}-e^{-\mu|x-y|}}{4 \mu^{3}}:=\frac{1}{4 \mu^{3}} F^{ \pm}(\mu|x-y|),
$$

and $\left(F^{ \pm}\right)^{\prime}(0)=0$, then by Lemma (2.5) (ii) and using the fact that $S_{0}(v)=S_{0}(x v(x))=0$, we have

$$
\begin{aligned}
{\left[R_{0}^{ \pm}\left(\mu^{4}\right)\right.} & \left.v\left(S_{0} A_{01}^{0} S_{0}\right) v R_{0}^{ \pm}\left(\mu^{4}\right)\right](x, y) \\
= & \frac{1}{16 \mu^{6}} \int_{\mathbf{R}^{2}} F^{ \pm}\left(\mu\left|x-y_{2}\right|\right)\left[v S_{0} A_{01}^{0} S_{0} v\right]\left(y_{2}, y_{1}\right) F^{ \pm}\left(\mu\left|y-y_{1}\right|\right) d y_{1} d y_{2} \\
= & \frac{1}{16 \mu^{2}} \int_{\mathbf{R}^{2}}\left(\int_{0}^{1} \int_{0}^{1}\left(1-\theta_{2}\right)\left(1-\theta_{1}\right)\left(F^{ \pm}\right)^{\prime \prime}\left(\mu\left|x-\theta_{2} y_{2}\right|\right) \times\right. \\
& \left.\left(F^{ \pm}\right)^{\prime \prime}\left(\mu\left|y-\theta_{1} y_{1}\right|\right) d \theta_{1} d \theta_{2}\right) y_{1}^{2} y_{2}^{2}\left[v S_{0} A_{01}^{0} S_{0} v\right]\left(y_{2}, y_{1}\right) d y_{1} d y_{2} .
\end{aligned}
$$

Then we obtain that

$$
\begin{aligned}
& K_{1, N}^{0, \pm}(t ; x, y) \\
= & \frac{1}{16} \int_{0}^{\infty} e^{-i t \mu^{4}} \mu \varphi_{0}\left(2^{-N} \mu\right)\left[\int _ { \mathbf { R } ^ { 2 } } \left(\int_{0}^{1} \int_{0}^{1}\left(1-\theta_{2}\right)\left(1-\theta_{1}\right)\left(F^{ \pm}\right)^{\prime \prime}\left(\mu\left|x-\theta_{2} y_{2}\right|\right)\right.\right. \\
& \left.\left.\left(F^{ \pm}\right)^{\prime \prime}\left(\mu\left|y-\theta_{1} y_{1}\right|\right) d \theta_{1} d \theta_{2}\right) y_{1}^{2} y_{2}^{2}\left[v S_{0} A_{01}^{0} S_{0} v\right]\left(y_{2}, y_{1}\right) d y_{1} d y_{2}\right] d \mu \\
= & \frac{1}{16} \int_{\mathbf{R}^{2}}\left[\int_{0}^{1} \int_{0}^{1}\left(\int_{0}^{\infty} e^{-i t \mu^{4}} \mu \varphi_{0}\left(2^{-N} \mu\right)\left(F^{ \pm}\right)^{\prime \prime}\left(\mu\left|x-\theta_{2} y_{2}\right|\right)\left(F^{ \pm}\right)^{\prime \prime}\left(\mu\left|y-\theta_{1} y_{1}\right|\right) d \mu\right)\right. \\
& \left.\left(1-\theta_{1}\right)\left(1-\theta_{2}\right) d \theta_{1} d \theta_{2}\right] y_{1}^{2} y_{2}^{2}\left[v S_{0} A_{01}^{0} S_{0} v\right]\left(y_{2}, y_{1}\right) d y_{1} d y_{2} .
\end{aligned}
$$


Denote

$$
\begin{aligned}
& E_{1, N}^{0, \pm}\left(t ; x, y, \theta_{1}, \theta_{2}, y_{1}, y_{2}\right) \\
= & \int_{0}^{\infty} e^{-i t \mu^{4}} \mu \varphi_{0}\left(2^{-N} \mu\right)\left(F^{ \pm}\right)^{\prime \prime}\left(\mu\left|x-\theta_{2} y_{2}\right|\right)\left(F^{ \pm}\right)^{\prime \prime}\left(\mu\left|y-\theta_{1} y_{1}\right|\right) d \mu .
\end{aligned}
$$

It is obvious that

$$
\left|K_{1, N}^{0, \pm}(t ; x, y)\right| \lesssim \int_{\mathbf{R}^{2}}\left(\int_{0}^{1} \int_{0}^{1}\left|E_{1, N}^{0, \pm}\left(t ; x, y, \theta_{1}, \theta_{2}, y_{1}, y_{2}\right)\right| d \theta_{1} d \theta_{2}\right)\left|\left[v S_{0} A_{0}^{0} S_{0} v\right]\left(y_{2}, y_{1}\right)\right| y_{1}^{2} y_{2}^{2} d y_{1} d y_{2} .
$$

Now we estimate $E_{1, N}^{0, \pm}\left(t ; x, y, \theta_{1}, \theta_{2}, y_{1}, y_{2}\right)$. Since

$$
\begin{aligned}
& \left(F^{ \pm}\right)^{\prime \prime}\left(\mu\left|x-\theta_{2} y_{2}\right|\right)=\mp i e^{ \pm i \mu\left|x-\theta_{2} y_{2}\right|}-e^{-\mu\left|x-\theta_{2} y_{2}\right|}:=e^{ \pm i \mu\left|x-\theta_{2} y_{2}\right|} \mathcal{F}^{ \pm}\left(\mu\left|x-\theta_{2} y_{2}\right|\right), \\
& \left(F^{ \pm}\right)^{\prime \prime}\left(\mu\left|y-\theta_{1} y_{1}\right|\right)=\mp i e^{ \pm i \mu\left|y-\theta_{1} y_{1}\right|}-e^{-\mu\left|y-\theta_{1} y_{1}\right|}:=e^{ \pm i \mu\left|y-\theta_{1} y_{1}\right|} \mathcal{F}^{ \pm}\left(\mu\left|y-\theta_{1} y_{1}\right|\right),
\end{aligned}
$$

and let $\mu=2^{N} s$, we can rewrite $E_{1, N}^{0, \pm}\left(t ; x, y, \theta_{1}, \theta_{2}, y_{1}, y_{2}\right)$ as follows:

$$
\begin{array}{r}
E_{1, N}^{0, \pm}\left(t ; x, y, \theta_{1}, \theta_{2}, y_{1}, y_{2}\right)=2^{2 N} \int_{0}^{\infty} e^{-i t 2^{4 N} s^{4}} s \varphi_{0}(s) e^{ \pm i 2^{N} s\left|x-\theta_{2} y_{2}\right|} \mathcal{F}^{ \pm}\left(2^{N} s\left|x-\theta_{2} y_{2}\right|\right) \\
\times e^{ \pm i 2^{N} s\left|y-\theta_{1} y_{1}\right|} \mathcal{F}^{ \pm}\left(2^{N} s\left|y-\theta_{1} y_{1}\right|\right) d s
\end{array}
$$

Noting that for any $s \in \operatorname{supp} \varphi_{0}(s)$ and $k=0,1$, we have

$$
\left|\partial_{s}^{k} \mathcal{F}^{ \pm}\left(2^{N} s\left|x-\theta_{2} y_{2}\right|\right)\right| \lesssim 1, \quad\left|\partial_{s}^{k} \mathcal{F}^{ \pm}\left(2^{N} s\left|y-\theta_{1} y_{1}\right|\right)\right| \lesssim 1
$$

Using Lemma 2.3 with $z=\left(x, y, y_{1}, y_{2}, \theta_{1}, \theta_{2}\right)$ and

$$
\Psi(z)=\left|x-\theta_{2} y_{2}\right|+\left|y-\theta_{1} y_{1}\right|, \quad \Phi^{ \pm}\left(2^{N} s ; z\right)=\mathcal{F}^{ \pm}\left(2^{N} s\left|x-\theta_{2} y_{2}\right|\right) \cdot \mathcal{F}^{ \pm}\left(2^{N} s\left|y-\theta_{1} y_{1}\right|\right),
$$

we obtain that

$$
\sup _{x, y \in \mathbf{R}}\left|E_{1, N}^{0, \pm}\left(t ; x, y, \theta_{1}, \theta_{2}, y_{1}, y_{2}\right)\right| \lesssim 2^{2 N}\left(1+|t| \cdot 2^{4 N}\right)^{-\frac{1}{2}}
$$

Noting that $v(x) \lesssim(1+|x|)^{-\frac{13}{2}-}$ in this case, thus we have

$$
\begin{aligned}
\left|K_{1, N}^{0, \pm}(t ; x, y)\right| & \lesssim 2^{2 N}\left(1+|t| \cdot 2^{4 N}\right)^{-\frac{1}{2}} \int_{\mathbf{R}^{2}} y_{2}^{2}\left|v\left(y_{2}\right)\right|\left|\left[S_{0} A_{0}^{0} S_{0}\right]\left(y_{2}, y_{1}\right)\right|\left|v\left(y_{1}\right)\right| y_{1}^{2} d y_{1} d y_{2} \\
& \lesssim 2^{2 N}\left(1+|t| \cdot 2^{4 N}\right)^{-\frac{1}{2}}\left\|y_{2}^{2} v\left(y_{2}\right)\right\|_{L_{y_{2}}^{2}} \cdot\left\|S_{0} A_{01}^{0} S_{0}\right\|_{L^{2} \rightarrow L_{2}} \cdot\left\|y_{1}^{2} v\left(y_{1}\right)\right\|_{L_{y_{1}}^{2}} \\
& \lesssim 2^{2 N}\left(1+|t| \cdot 2^{4 N}\right)^{-\frac{1}{2}}
\end{aligned}
$$

uniformly in $x, y$.

Proposition 2.8. For any $N \in \mathbf{Z}_{-}$and $N \leq N_{0}$, we have

$$
\sup _{x, y \in \mathbf{R}}\left|\int_{0}^{\infty} e^{-i t \mu^{4}} \mu^{3} \varphi_{N}(\mu)\left[R_{0}^{ \pm}\left(\mu^{4}\right) v\left(\mu Q A_{11}^{0} Q\right) v R_{0}^{ \pm}\left(\mu^{4}\right)\right](x, y) d \mu\right| \lesssim 2^{N}\left(1+|t| \cdot 2^{4 N}\right)^{-\frac{1}{2}} .
$$

Proof. Since $\varphi_{N}(\mu)=\varphi_{0}\left(2^{-N} \mu\right)$, thus

$$
K_{2, N}^{0, \pm}(t ; x, y):=\int_{0}^{\infty} e^{-i t \mu^{4}} \mu^{3} \varphi_{0}\left(2^{-N} \mu\right)\left[R_{0}^{ \pm}\left(\mu^{4}\right) v\left(\mu Q A_{11}^{0} Q\right) v R_{0}^{ \pm}\left(\mu^{4}\right)\right](x, y) d \mu .
$$

It suffices to prove that

$$
\sup _{x, y}\left|K_{2, N}^{0, \pm}(t ; x, y)\right| \lesssim 2^{N}\left(1+|t| \cdot 2^{4 N}\right)^{-\frac{1}{2}}
$$

Indeed, since

$$
R_{0}^{ \pm}(x, y)=\frac{ \pm i e^{ \pm i \mu|x-y|}-e^{-\mu|x-y|}}{4 \mu^{3}}:=\frac{1}{4 \mu^{3}} F^{ \pm}(\mu|x-y|),
$$


by Lemma (2.5) (i) and using the fact that $Q(v)=0$, we have

$$
\begin{aligned}
& {\left[R_{0}^{ \pm}\left(\mu^{4}\right) v\left(\mu Q A_{11}^{0} Q\right) v R_{0}^{ \pm}\left(\mu^{4}\right)\right](x, y) } \\
= & \frac{1}{16 \mu^{5}} \int_{\mathbf{R}^{2}} F\left(\mu\left|x-y_{2}\right|\right)\left[v Q A_{11}^{0} Q v\right]\left(y_{2}, y_{1}\right) F\left(\mu\left|y-y_{1}\right|\right) d y_{1} d y_{2} \\
= & \frac{1}{16 \mu^{3}} \int_{\mathbf{R}^{2}}\left(\int_{0}^{1} \int_{0}^{1}\left(F^{ \pm}\right)^{\prime}\left(\mu\left|x-\theta_{2} y_{2}\right|\right) \operatorname{sgn}\left(x-\theta_{2} y_{2}\right)\left[v Q A_{11}^{0} Q v\right]\left(y_{2}, y_{1}\right)\right. \\
& \left.\left(F^{ \pm}\right)^{\prime}\left(\mu\left|y-\theta_{1} y_{1}\right|\right) \operatorname{sgn}\left(y-\theta_{1} y_{1}\right) d \theta_{1} d \theta_{2}\right) y_{1} y_{2} d y_{1} d y_{2} .
\end{aligned}
$$

Then we obtain that

$$
\begin{aligned}
& K_{2, N}^{0, \pm}(t ; x, y) \\
&= \frac{1}{16} \int_{\mathbf{R}^{2}}\left[\int _ { 0 } ^ { 1 } \int _ { 0 } ^ { 1 } \operatorname { s g n } ( y - \theta _ { 1 } y _ { 1 } ) \operatorname { s g n } ( x - \theta _ { 2 } y _ { 2 } ) \left(\int_{0}^{\infty} e^{-i t \mu^{4}} \varphi_{0}\left(2^{-N} \mu\right)\left(F^{ \pm}\right)^{\prime}\left(\mu\left|x-\theta_{2} y_{2}\right|\right)\right.\right. \\
&\left.\left.\left(F^{ \pm}\right)^{\prime}\left(\mu\left|y-\theta_{1} y_{1}\right|\right) d \mu\right) d \theta_{1} d \theta_{2}\right]\left[v Q A_{11}^{0} Q v\right]\left(y_{2}, y_{1}\right) y_{1} y_{2} d y_{1} d y_{2} .
\end{aligned}
$$

Denote

$$
E_{2, N}^{0, \pm}\left(t ; x, y, \theta_{1}, \theta_{2}, y_{1}, y_{2}\right)=\int_{0}^{\infty} e^{-i t \mu^{4}} \varphi_{0}\left(2^{-N} \mu\right)\left(F^{ \pm}\right)^{\prime}\left(\mu\left|x-\theta_{2} y_{2}\right|\right)\left(F^{ \pm}\right)^{\prime}\left(\mu\left|y-\theta_{1} y_{1}\right|\right) d \mu .
$$

Then

$$
\left|K_{2, N}^{0, \pm}(t ; x, y)\right| \lesssim \int_{\mathbf{R}^{2}}\left(\int_{0}^{1} \int_{0}^{1}\left|E_{2, N}^{0, \pm}\left(t ; x, y, \theta_{1}, \theta_{2}, y_{1}, y_{2}\right)\right| d \theta_{1} d \theta_{2}\right)\left|\left[v Q A_{11}^{0} Q v\right]\right|\left(y_{2}, y_{1}\right)\left|y_{1} y_{2}\right| d y_{1} d y_{2} \text {. }
$$

Next to estimate $E_{2, N}^{0, \pm}\left(t ; x, y, \theta_{1}, \theta_{2}, y_{1}, y_{2}\right)$. Since

$$
\begin{aligned}
& \left(F^{ \pm}\right)^{\prime}\left(\mu\left|x-\theta_{2} y_{2}\right|\right)=-e^{ \pm i \mu\left|x-\theta_{2} y_{2}\right|}+e^{-\mu\left|x-\theta_{2} y_{2}\right|}:=e^{ \pm i \mu\left|x-\theta_{2} y_{2}\right|} \mathcal{F}^{ \pm}\left(\mu\left|x-\theta_{2} y_{2}\right|\right), \\
& \left(F^{ \pm}\right)^{\prime}\left(\mu\left|y-\theta_{1} y_{1}\right|\right)=-e^{ \pm i \mu\left|y-\theta_{1} y_{1}\right|}+e^{-\mu\left|y-\theta_{1} y_{1}\right|}:=e^{ \pm i \mu\left|y-\theta_{1} y_{1}\right|} \mathcal{F}^{ \pm}\left(\mu\left|y-\theta_{1} y_{1}\right|\right),
\end{aligned}
$$

and let $\mu=2^{N} s$, we can rewrite $E_{2, N}^{0, \pm}\left(t ; x, y, \theta_{1}, \theta_{2}, y_{1}, y_{2}\right)$ as follows:

$$
\begin{array}{r}
E_{2, N}^{0, \pm}\left(t ; x, y, \theta_{1}, \theta_{2}, y_{1}, y_{2}\right)=2^{N} \int_{0}^{\infty} e^{-i t 2^{4 N} s^{4}} \varphi_{0}(s) e^{ \pm i 2^{N} s\left|x-\theta_{2} y_{2}\right|} \mathcal{F}^{ \pm}\left(2^{N} s\left|x-\theta_{2} y_{2}\right|\right) \\
e^{ \pm i 2^{N} s\left|y-\theta_{1} y_{1}\right|} \mathcal{F}^{ \pm}\left(2^{N} s\left|y-\theta_{1} y_{1}\right|\right) d s
\end{array}
$$

Noting that for any $s \in \operatorname{supp} \varphi_{0}(s)$ and $k=0,1$, we have

$$
\left|\partial_{s}^{k} \mathcal{F}^{ \pm}\left(2^{N} s\left|x-\theta_{2} y_{2}\right|\right)\right| \lesssim 1, \quad\left|\partial_{s}^{k} \mathcal{F}^{ \pm}\left(2^{N} s\left|y-\theta_{1} y_{1}\right|\right)\right| \lesssim 1
$$

then using Lemma 2.3 with $z=\left(x, y, y_{1}, y_{2}, \theta_{1}, \theta_{2}\right)$ and

$$
\Psi(z)=\left|x-\theta_{2} y_{2}\right|+\left|y-\theta_{1} y_{1}\right|, \quad \Phi^{ \pm}\left(2^{N} s ; z\right)=\mathcal{F}^{ \pm}\left(2^{N} s\left|x-\theta_{2} y_{2}\right|\right) \cdot \mathcal{F}^{ \pm}\left(2^{N} s\left|y-\theta_{1} y_{1}\right|\right),
$$

we have

$$
\sup _{x, y \in \mathbf{R}}\left|E_{2, N}^{0, \pm}\left(t ; x, y, \theta_{1}, \theta_{2}, y_{1}, y_{2}\right)\right| \lesssim 2^{N}\left(1+|t| \cdot 2^{4 N}\right)^{-\frac{1}{2}}
$$

Furthermore, since $v(x) \lesssim(1+|x|)^{-\frac{13}{2}-}$, we have

$$
\begin{aligned}
\left|K_{2, N}^{0, \pm}(t ; x, y)\right| & \lesssim 2^{N}\left(1+|t| \cdot 2^{4 N}\right)^{-\frac{1}{2}} \int_{\mathbf{R}^{2}}\left|y_{2} v\left(y_{2}\right)\right|\left|\left[Q A_{11}^{0} Q\right]\left(y_{2}, y_{1}\right)\right|\left|y_{1} v\left(y_{1}\right)\right| d y_{1} d y_{2} \\
& \lesssim 2^{N}\left(1+|t| \cdot 2^{4 N}\right)^{-\frac{1}{2}}\left\|y_{2} v\left(y_{2}\right)\right\|_{L_{y_{2}}^{2}} \cdot\left\|Q A_{11}^{0} Q\right\|_{L^{2} \rightarrow L_{2}} \cdot\left\|y_{1} v\left(y_{1}\right)\right\|_{L_{y_{1}}^{2}} \\
& \lesssim 2^{N}\left(1+|t| \cdot 2^{4 N}\right)^{-\frac{1}{2}}
\end{aligned}
$$


uniformly in $x, y$.

By using the method in the proof of Proposition 2.7 and Proposition 2.8, it is easy similarly to obtain the following estimates ( we omit the details of calculations here ).

Proposition 2.9. For any $N \in \mathbf{Z}_{-}$and $N \leq N_{0}$, we have

$$
\begin{aligned}
& \sup _{x, y}\left|\int_{0}^{\infty} e^{-i t \mu^{4}} \mu^{3} \varphi_{N}(\mu)\left[R_{0}^{ \pm}\left(\mu^{4}\right) v\left(\mu^{2} Q A_{21}^{0} Q\right) v R_{0}^{ \pm}\left(\mu^{4}\right)\right](x, y) d \mu\right| \lesssim 2^{2 N}\left(1+|t| \cdot 2^{4 N}\right)^{-\frac{1}{2}} . \\
& \sup _{x, y}\left|\int_{0}^{\infty} e^{-i t \mu^{4}} \mu^{3} \varphi_{N}(\mu)\left[R_{0}^{ \pm}\left(\mu^{4}\right) v\left(\mu^{2} S_{0} A_{22}^{0}\right) v R_{0}^{ \pm}\left(\mu^{4}\right)\right](x, y) d \mu\right| \lesssim 2^{2 N}\left(1+|t| \cdot 2^{4 N}\right)^{-\frac{1}{2}} . \\
& \sup _{x, y}\left|\int_{0}^{\infty} e^{-i t \mu^{4}} \mu^{3} \varphi_{N}(\mu)\left[R_{0}^{ \pm}\left(\mu^{4}\right) v\left(\mu^{2} A_{23}^{0} S_{0}\right) v R_{0}^{ \pm}\left(\mu^{4}\right)\right](x, y) d \mu\right| \lesssim 2^{2 N}\left(1+|t| \cdot 2^{4 N}\right)^{-\frac{1}{2}} .
\end{aligned}
$$

Finally, for the last remained term $R_{0}^{ \pm}\left(\mu^{4}\right) v \Gamma_{3}^{0}(\mu) v R_{0}^{ \pm}\left(\mu^{4}\right)$ in (2.25), we have the following estimates.

Proposition 2.10. For any $N \in \mathbf{Z}_{-}$and $N \leq N_{0}$, we have

$$
\sup _{x, y}\left|\int_{0}^{\infty} e^{-i t \mu^{4}} \mu^{3} \varphi_{N}(\mu)\left[R_{0}^{ \pm}\left(\mu^{4}\right) v \Gamma_{3}^{0}(\mu) v R_{0}^{ \pm}\left(\mu^{4}\right)\right](x, y) d \mu\right| \lesssim 2^{N}\left(1+|t| \cdot 2^{4 N}\right)^{-\frac{1}{2}}
$$

Proof. Indeed, it suffices to prove that for any $f, g \in L^{1}(\mathbf{R})$,

$$
\left|\int_{0}^{\infty} e^{-i t \mu^{4}} \mu^{3} \varphi_{N}(\mu)\left\langle\left[v \Gamma_{3}^{0}(\mu) v\right] R_{0}^{ \pm}\left(\mu^{4}\right) f,\left(R_{0}^{ \pm}\right)^{*}\left(\mu^{4}\right) g\right\rangle d \mu\right| \lesssim 2^{N}\left(1+|t| \cdot 2^{4 N}\right)^{-\frac{1}{2}}\|f\|_{L^{1}} \cdot\|g\|_{L^{1}} .
$$

Equivalently, it is enough to bound the following kernel uniformly by $O\left(2^{N}\left(1+|t| \cdot 2^{4 N}\right)^{-\frac{1}{2}}\right)$ :

$$
K_{4, N}^{0, \pm}(t ; x, y)=\int_{0}^{\infty} e^{-i t \mu^{4}} \mu^{3} \varphi_{N}(\mu)\left\langle\left[v \Gamma_{3}^{0}(\mu) v\right]\left(R_{0}^{ \pm}\left(\mu^{4}\right)(*, y)\right)(\cdot), R_{0}^{\mp}\left(\mu^{4}\right)(x, \cdot)\right\rangle d \mu
$$

Noting that

$$
R_{0}^{ \pm}\left(\mu^{4}\right)(x, y)=\frac{1}{4 \mu^{3}}\left( \pm i e^{ \pm i \mu|x-y|}-e^{-\mu|x-y|}\right):=\frac{e^{ \pm i \mu|x-y|}}{4 \mu^{3}} \mathcal{R}_{0}^{ \pm}(\mu)(x, y),
$$

thus, we have

$$
\begin{aligned}
& \left\langle\left[v \Gamma_{3}^{0}(\mu) v\right]\left(R_{0}^{ \pm}\left(\mu^{4}\right)(*, y)\right)(\cdot), R_{0}^{\mp}\left(\mu^{4}\right)(x, \cdot)\right\rangle \\
= & \frac{1}{16 \mu^{6}}\left\langle\left[v \Gamma_{3}^{0}(\mu) v\right]\left(e^{ \pm i \mu|*-y|} \mathcal{R}_{0}^{ \pm}(\mu)(*, y)\right)(\cdot),\left(e^{\mp i \mu|x-\cdot|} \mathcal{R}_{0}^{\mp}(\mu)(x, \cdot)\right)\right\rangle \\
= & \frac{1}{16 \mu^{6}} e^{ \pm i \mu|x|} e^{ \pm i \mu|y|}\left\langle\left[v \Gamma_{3}^{0}(\mu) v\right]\left(e^{ \pm i \mu(|*-y|-|y|)} \mathcal{R}_{0}^{ \pm}(\mu)(*, y)\right)(\cdot),\left(e^{\mp i \mu(|x-\cdot|-|x|)} \mathcal{R}_{0}^{\mp}(\mu)(x, \cdot)\right)\right\rangle \\
:= & \frac{1}{16 \mu^{6}} e^{ \pm i \mu(|x|+|y|)} E_{3, N}^{0, \pm}(\mu ; x, y) .
\end{aligned}
$$

Let $\mu=2^{N} s$, we have

$$
K_{4, N}^{0, \pm}(t ; x, y)=2^{-2 N} \int_{0}^{\infty} e^{-i t 2^{4 N} s^{4}} \varphi_{0}(s) s^{-3} e^{ \pm i 2^{N} s(|x|+|y|)} E_{3, N}^{0, \pm}\left(2^{N} s ; x, y\right) d s .
$$

Noting that for any $s \in \operatorname{supp} \varphi_{0}(s)$ and any $N \in \mathbf{Z}_{-}$, we have

$$
\begin{aligned}
& \left|\partial_{s}^{k}\left(e^{ \pm i 2^{N} s(*-y|-| y \mid)} \mathcal{R}_{0}^{ \pm}\left(2^{N} s\right)(*, y)\right)\right| \lesssim\langle *\rangle^{k}, \quad k=0,1, \\
& \left|\partial_{s}^{k}\left(e^{ \pm i 2^{N} s(|x-\cdot|-|x|)} \mathcal{R}_{0}^{ \pm}\left(2^{N} s\right)(x, \cdot)\right)\right| \lesssim\langle\cdot\rangle^{k}, \quad k=0,1,
\end{aligned}
$$


and since $\left\|\Gamma_{3}^{0}(\mu)\right\|_{L^{2} \rightarrow L^{2}}=O_{1}\left(\mu^{3}\right)$ for $\mu<<1$ from Theorem 1.8 ( e.g. choosing $\mu<2^{N_{0}}$ for some large $N_{0} \in \mathbf{Z}_{-}$), then we have

$$
\left\|\partial_{s}^{k}\left(\Gamma_{3}^{0}\left(2^{N} s\right)\right)\right\|_{L^{2} \rightarrow L^{2}} \lesssim 2^{3 N} s^{3-k}, \quad k=0,1 .
$$

Furthermore, since

$$
\begin{aligned}
& \partial_{s} E_{3, N}^{0, \pm}\left(2^{N} s ; x, y\right) \\
= & \left\langle\left[v \partial_{s} \Gamma_{3}^{0}\left(2^{N} s\right) v\right]\left(e^{ \pm i 2^{N} s(|*-y|-|y|)} \mathcal{R}_{0}^{ \pm}\left(2^{N} s\right)(*, y)\right)(\cdot),\left(e^{\mp i 2^{N} s(|x-\cdot|-|x|)} \mathcal{R}_{0}^{\mp}\left(2^{N} s\right)(x, \cdot)\right)\right\rangle \\
& +\left\langle\left[v \Gamma_{3}^{0}\left(2^{N} s\right) v\right] \partial_{s}\left(e^{ \pm i 2^{N} s(|*-y|-|y|)} \mathcal{R}_{0}^{ \pm}\left(2^{N} s\right)(*, y)\right)(\cdot),\left(e^{\mp i 2^{N} s(|x-\cdot|-|x|)} \mathcal{R}_{0}^{\mp}\left(2^{N} s\right)(x, \cdot)\right)\right\rangle \\
& +\left\langle\left[v \Gamma_{3}^{0}\left(2^{N} s\right) v\right]\left(e^{ \pm i 2^{N} s(|*-y|-|y|)} \mathcal{R}_{0}^{ \pm}\left(2^{N} s\right)(*, y)\right)(\cdot), \partial_{s}\left(e^{\mp i 2^{N} s(|x-\cdot|-|x|)} \mathcal{R}_{0}^{\mp}\left(2^{N} s\right)(x, \cdot)\right)\right\rangle,
\end{aligned}
$$

and $v(x) \lesssim(1+|x|)^{-\frac{13}{2}-}$, then by Hölder inequality, for any $s \in \operatorname{supp} \varphi_{0}(s)$, we have

$$
\left|\partial_{s}^{k} E_{3, N}^{0, \pm}\left(2^{N} s ; x, y\right)\right| \lesssim 2^{3 N} s^{3-k}, \quad k=0,1 .
$$

Using again Lemma 2.3 with $z=(x, y)$, and

$$
\Psi(z)=|x|+|y|, \quad \Phi^{ \pm}\left(2^{N} s ; z\right)=2^{-3 N} E_{3, N}^{0, \pm}\left(2^{N} s ; x, y\right)
$$

we have

$$
\sup _{x, y \in \mathbf{R}}\left|K_{4, N}^{0, \pm}(t ; x, y)\right| \lesssim 2^{N}\left(1+|t| \cdot 2^{4 N}\right)^{-\frac{1}{2}}
$$

2.2.2. First Kind of Resonance. If there is first kind of resonance at zero, using (1.20) and (1.22), we have

$$
\begin{aligned}
R_{V}^{ \pm}\left(\mu^{4}\right)= & R_{0}^{ \pm}\left(\mu^{4}\right)-R_{0}^{ \pm}\left(\mu^{4}\right) v\left(\mu^{-1} S_{1} A_{-1,1}^{1} S_{1}\right) v R_{0}^{ \pm}\left(\mu^{4}\right)-R_{0}^{ \pm}\left(\mu^{4}\right) v\left(S_{0} A_{01}^{1} Q+Q A_{02}^{1} S_{0}\right) v R_{0}^{ \pm}\left(\mu^{4}\right) \\
& -R_{0}^{ \pm}\left(\mu^{4}\right) v\left(\mu Q A_{11}^{1} Q\right) v R_{0}^{ \pm}\left(\mu^{4}\right)-R_{0}^{ \pm}\left(\mu^{4}\right) v\left(\mu S_{0} A_{12}^{1}+\mu A_{13}^{1} S_{0}\right) v R_{0}^{ \pm}\left(\mu^{4}\right) \\
& -R_{0}^{ \pm}\left(\mu^{4}\right) v\left(\mu^{2} Q A_{21}^{1}+\mu^{2} A_{22}^{1} Q\right) v R_{0}^{ \pm}\left(\mu^{4}\right)-R_{0}^{ \pm}\left(\mu^{4}\right) v \Gamma_{3}^{1}(\mu) v R_{0}^{ \pm}\left(\mu^{4}\right)
\end{aligned}
$$

By checking the analysis of Regular Case, we only need to establish the following two propositions.

Proposition 2.11. For any $N \in \mathbf{Z}_{-}$and $N \leq N_{0}$, we have

$$
\sup _{x, y}\left|\int_{0}^{\infty} e^{-i t \mu^{4}} \mu^{3} \varphi_{N}(\mu)\left[R_{0}^{ \pm}\left(\mu^{4}\right) v\left(\mu^{-1} S_{1} A_{-1}^{1} S_{1}\right) v R_{0}^{ \pm}\left(\mu^{4}\right)\right](x, y) d \mu\right| \lesssim 2^{N}\left(1+|t| \cdot 2^{4 N}\right)^{-\frac{1}{2}}
$$

Proof. Since $S_{1}$ has the same orthogonality relationships with $S_{0}$, thus the proof is the same as the proof of Proposition 2.7.

According to the orthogonality of $S_{1}$ and $Q$, using Lemma 2.5, it is easy to prove the following estimates.

Proposition 2.12. For any $N \in \mathbf{Z}_{-}$and $N \leq N_{0}$, we have

$$
\begin{aligned}
& \sup _{x, y}\left|\int_{0}^{\infty} e^{-i t \mu^{4}} \mu^{3} \varphi_{N}(\mu)\left[R_{0}^{ \pm}\left(\mu^{4}\right) v\left(S_{0} A_{01}^{1} Q\right) v R_{0}^{ \pm}\left(\mu^{4}\right)\right](x, y) d \mu\right| \lesssim 2^{N}\left(1+|t| \cdot 2^{4 N}\right)^{-\frac{1}{2}} . \\
& \sup _{x, y}\left|\int_{0}^{\infty} e^{-i t \mu^{4}} \mu^{3} \varphi_{N}(\mu)\left[R_{0}^{ \pm}\left(\mu^{4}\right) v\left(Q A_{01}^{1} S_{0}\right) v R_{0}^{ \pm}\left(\mu^{4}\right)\right](x, y) d \mu\right| \lesssim 2^{N}\left(1+|t| \cdot 2^{4 N}\right)^{-\frac{1}{2}} .
\end{aligned}
$$


2.2.3. Second Kind of Resonance. If there is second kind of resonance at zero, using (1.20) and (1.23), we have

$$
\begin{aligned}
& R_{V}^{ \pm}\left(\mu^{4}\right)= \\
& R_{0}^{ \pm}\left(\mu^{4}\right)-R_{0}^{ \pm}\left(\mu^{4}\right) v\left(\mu^{-3} S_{2} A_{-3,1}^{2} S_{2}\right) v R_{0}^{ \pm}\left(\mu^{4}\right)-R_{0}^{ \pm}\left(\mu^{4}\right) v \mu^{-2}\left(S_{2} A_{-2,1}^{2} S_{0}+S_{0} A_{-2,2}^{2} S_{2}\right) v R_{0}^{ \pm}\left(\mu^{4}\right) \\
& \quad-R_{0}^{ \pm}\left(\mu^{4}\right) v\left(\mu^{-1} S_{0} A_{-1,1}^{2} S_{0}\right) v R_{0}^{ \pm}\left(\mu^{4}\right)-R_{0}^{ \pm}\left(\mu^{4}\right) v\left(\mu^{-1} S_{2} A_{-1,2}^{2} Q+\mu^{-1} Q A_{-1,2}^{2} S_{2}\right) v R_{0}^{ \pm}\left(\mu^{4}\right) \\
& \quad-R_{0}^{ \pm}\left(\mu^{4}\right) v\left(Q A_{01}^{2} S_{0}+S_{0} A_{02}^{2} Q\right) v R_{0}^{ \pm}\left(\mu^{4}\right)-R_{0}^{ \pm}\left(\mu^{4}\right) v\left(S_{2} A_{03}^{2}+A_{04}^{2} S_{2}\right) v R_{0}^{ \pm}\left(\mu^{4}\right) \\
& \quad-R_{0}^{ \pm}\left(\mu^{4}\right) v\left(\mu Q A_{11}^{2} Q\right) v R_{0}^{ \pm}\left(\mu^{4}\right)-R_{0}^{ \pm}\left(\mu^{4}\right) v\left(\mu S_{0} A_{12}^{2}+\mu A_{13}^{2} S_{0}\right) v R_{0}^{ \pm}\left(\mu^{4}\right) \\
& \quad-R_{0}^{ \pm}\left(\mu^{4}\right) v\left(\mu^{2} Q A_{21}^{2}+\mu^{2} A_{22}^{2} Q\right) v R_{0}^{ \pm}\left(\mu^{4}\right)-R_{0}^{ \pm}\left(\mu^{4}\right) v \Gamma_{3}^{2}(\mu) v R_{0}^{ \pm}\left(\mu^{4}\right) .
\end{aligned}
$$

By combing the analysis of Regular Case and First Kind of Resonance, it is suffices to establish the following estimates.

Proposition 2.13. For any $N \in \mathbf{Z}_{-}$and $N \leq N_{0}$, we have

$$
\begin{aligned}
& \sup _{x, y}\left|\int_{0}^{\infty} e^{-i t \mu^{4}} \varphi_{N}(\mu) \mu^{3}\left[R_{0}^{ \pm}\left(\mu^{4}\right) v\left(\mu^{-3} S_{2} A_{-3,1}^{2} S_{2}\right) v R_{0}^{ \pm}\left(\mu^{4}\right)\right](x, y) d \mu\right| \lesssim 2^{N}\left(1+|t| \cdot 2^{4 N}\right)^{-\frac{1}{2}}, \\
& \sup _{x, y}\left|\int_{0}^{\infty} e^{-i t \mu^{4}} \varphi_{N}(\mu) \mu^{3}\left[R_{0}^{ \pm}\left(\mu^{4}\right) v\left(\mu^{-2} S_{2} A_{-2,1}^{2} S_{0}\right) v R_{0}^{ \pm}\left(\mu^{4}\right)\right](x, y) d \mu\right| \lesssim 2^{N}\left(1+|t| \cdot 2^{4 N}\right)^{-\frac{1}{2}} . \\
& \sup _{x, y}\left|\int_{0}^{\infty} e^{-i t \mu^{4}} \varphi_{N}(\mu) \mu^{3}\left[R_{0}^{ \pm}\left(\mu^{4}\right) v\left(\mu^{-2} S_{0} A_{-2,2}^{2} S_{2}\right) v R_{0}^{ \pm}\left(\mu^{4}\right)\right](x, y) d \mu\right| \lesssim 2^{N}\left(1+|t| \cdot 2^{4 N}\right)^{-\frac{1}{2}} .
\end{aligned}
$$

Proof. We only prove (2.36) here since the other two estimates can be proved by the similar way. Note that

$$
K_{1, N}^{2, \pm}(t ; x, y):=\int_{0}^{\infty} e^{-i t \mu^{4}} \varphi_{N}(\mu) \mu^{3}\left[R_{0}^{ \pm}\left(\mu^{4}\right) v\left(\mu^{-3} S_{2} A_{-3,1}^{2} S_{2}\right) v R_{0}^{ \pm}\left(\mu^{4}\right)\right](x, y) d \mu .
$$

It suffices to prove that

$$
\sup _{x, y}\left|K_{1, N}^{2, \pm}(t ; x, y)\right| \lesssim 2^{N}\left(1+|t| \cdot 2^{4 N}\right)^{-\frac{1}{2}}
$$

Noting that

$$
R_{0}^{ \pm}(x, y)=\frac{ \pm i e^{ \pm i \mu|x-y|}-e^{-\mu|x-y|}}{4 \mu^{3}}
$$

by Lemma 2.5(iii) and using the fact that $S_{2}(v)=S_{2}(x v(x))=S_{2}\left(x^{2} v(x)\right)=0$, we have

$$
\begin{aligned}
& {\left[R_{0}^{ \pm}\left(\mu^{4}\right) v\left(\mu^{-3} S_{2} A_{-3,1}^{2} S_{2}\right) v R_{0}^{ \pm}\left(\mu^{4}\right)\right](x, y) } \\
= & \frac{1}{16 \mu^{9}} \int_{\mathbf{R}^{2}}\left( \pm i e^{ \pm i \mu\left|x-y_{2}\right|}-e^{-\mu\left|x-y_{2}\right|}+\frac{1 \pm i}{2} \mu^{2}\left|x-y_{2}\right|^{2}\right)\left[v S_{2} A_{-3,1}^{2} S_{2} v\right]\left(y_{2}, y_{1}\right) \\
& \left( \pm i e^{ \pm i \mu\left|y-y_{1}\right|}-e^{-\mu\left|y-y_{1}\right|}+\frac{1 \pm i}{2} \mu^{2}\left|y-y_{1}\right|^{2}\right) d y_{1} d y_{2} \\
:= & \frac{1}{16 \mu^{9}} \int_{\mathbf{R}^{2}} F^{ \pm}\left(\mu\left|x-y_{2}\right|\right)\left[v S_{2} A_{-3,1}^{2} S_{2} v\right]\left(y_{2}, y_{1}\right) F^{ \pm}\left(\mu\left|y-y_{1}\right|\right) d y_{1} d y_{2},
\end{aligned}
$$

where $F^{ \pm}(p)= \pm i e^{ \pm i p}-e^{-p}+\frac{1 \pm i}{2} p^{2}$. 
It is obvious that $F^{ \pm}(p) \in C^{\infty}(\mathbf{R})$ and $\left(F^{ \pm}\right)^{(k)}(0)=0(k=1,2)$, then by (2.17) and using the fact that $S_{2}(v)=S_{2}(x v(x))=S_{2}\left(x^{2} v(x)\right)=0$, we obtain that

$$
\begin{aligned}
& {\left[R_{0}^{ \pm}\left(\mu^{4}\right) v\left(\mu^{-3,1} S_{2} A_{-3}^{2} S_{2}\right) v R_{0}^{ \pm}\left(\mu^{4}\right)\right](x, y) } \\
= & \frac{1}{16 \mu^{9}} \int_{\mathbf{R}^{2}} F^{ \pm}\left(\mu\left|x-y_{2}\right|\right)\left[v S_{2} A_{-3,1}^{2} S_{2} v\right]\left(y_{2}, y_{1}\right) F^{ \pm}\left(\mu\left|y-y_{1}\right|\right) d y_{1} d y_{2} \\
= & \frac{1}{16(2 !)^{2} \mu^{3}} \int_{\mathbf{R}^{2}}\left(\int_{0}^{1} \int_{0}^{1}\left(1-\theta_{1}\right)^{2}\left(1-\theta_{2}\right)^{2}\left(F^{ \pm}\right)^{(3)}\left(\mu\left|x-\theta_{2} y_{2}\right|\right)\left(\operatorname{sgn}\left(x-\theta_{2} y_{2}\right)\right)^{3}\right. \\
& \left.\left(F^{ \pm}\right)^{(3)}\left(\mu\left|y-\theta_{1} y_{1}\right|\right)\left(\operatorname{sgn}\left(y-\theta_{1} y_{1}\right)\right)^{3} d \theta_{1} d \theta_{2}\right) y_{1}^{3} y_{2}^{3}\left[v S_{2} A_{-3,1}^{2} S_{2} v\right]\left(y_{2}, y_{1}\right) d y_{1} d y_{2} .
\end{aligned}
$$

Then we obtain that

$$
\begin{aligned}
& K_{1, N}^{2, \pm}(t ; x, y) \frac{1}{16(2 !)^{2}} \int_{\mathbf{R}^{2}}\left[\int _ { 0 } ^ { 1 } \int _ { 0 } ^ { 1 } ( 1 - \theta _ { 1 } ) ^ { 2 } ( 1 - \theta _ { 2 } ) ^ { 2 } ( \operatorname { s g n } ( y - \theta _ { 1 } y _ { 1 } ) ) ^ { 3 } ( \operatorname { s g n } ( x - \theta _ { 2 } y _ { 2 } ) ) ^ { 3 } \left(\int_{0}^{\infty} e^{-i t \mu^{4}} \varphi_{0}\left(2^{-N} \mu\right)\right.\right. \\
&\left.\left.\left(F^{ \pm}\right)^{(3)}\left(\mu\left|x-\theta_{2} y_{2}\right|\right)\left(F^{ \pm}\right)^{(3)}\left(\mu\left|y-\theta_{1} y_{1}\right|\right) d \mu\right) d \theta_{1} d \theta_{2}\right] y_{1}^{3} y_{2}^{3}\left[v S_{2} A_{-3,1}^{2} S_{2} v\right]\left(y_{2}, y_{1}\right) d y_{1} d y_{2} .
\end{aligned}
$$

Denote

$$
E_{1, N}^{2, \pm}\left(t ; x, y, \theta_{1}, \theta_{2}, y_{1}, y_{2}\right)=\int_{0}^{\infty} e^{-i t \mu^{4}} \varphi_{0}\left(2^{-N} \mu\right)\left(F^{ \pm}\right)^{(3)}\left(\mu\left|x-\theta_{2} y_{2}\right|\right)\left(F^{ \pm}\right)^{(3)}\left(\mu\left|y-\theta_{1} y_{1}\right|\right) d \mu .
$$

Thus

$$
\left|K_{1, N}^{2, \pm}(t ; x, y)\right| \lesssim \int_{\mathbf{R}^{2}}\left(\int_{0}^{1} \int_{0}^{1}\left|E_{1, N}^{2, \pm}\left(t ; x, y, \theta_{1}, \theta_{2}, y_{1}, y_{2}\right)\right| d \theta_{1} d \theta_{2}\right)\left|\left[v S_{2} A_{-3,1}^{2} S_{2} v\right]\left(y_{2}, y_{1}\right)\right|\left|y_{1}^{3} y_{2}^{3}\right| d y_{1} d y_{2} .
$$

Now we consider $E_{1, N}^{2, \pm}\left(t ; x, y, \theta_{1}, \theta_{2}, y_{1}, y_{2}\right)$. Since

$$
\begin{aligned}
& \left(F^{ \pm}\right)^{(3)}\left(\mu\left|x-\theta_{2} y_{2}\right|\right)=e^{ \pm i \mu\left|x-\theta_{2} y_{2}\right|}+e^{-\mu\left|x-\theta_{2} y_{2}\right|}:=e^{ \pm i \mu\left|x-\theta_{2} y_{2}\right|} \mathcal{F}^{ \pm}\left(\mu\left|x-\theta_{2} y_{2}\right|\right), \\
& \left(F^{ \pm}\right)^{(3)}\left(\mu\left|y-\theta_{1} y_{1}\right|\right)=e^{ \pm i \mu\left|y-\theta_{1} y_{1}\right|}+e^{-\mu\left|y-\theta_{1} y_{1}\right|}:=e^{ \pm i \mu\left|y-\theta_{1} y_{1}\right|} \mathcal{F}^{ \pm}\left(\mu\left|y-\theta_{1} y_{1}\right|\right),
\end{aligned}
$$

let $\mu=2^{N} s$, we can rewrite $E_{1, N}^{2, \pm}\left(t ; x, y, \theta_{1}, \theta_{2}, y_{1}, y_{2}\right)$ as follows:

$$
\begin{array}{r}
E_{1, N}^{2, \pm}\left(t ; x, y, \theta_{1}, \theta_{2}, y_{1}, y_{2}\right)=2^{N} \int_{0}^{\infty} e^{-i t 2^{4 N} s^{4}} \varphi_{0}(s) e^{ \pm i 2^{N} s\left|x-\theta_{2} y_{2}\right|} \mathcal{F}^{ \pm}\left(2^{N} s\left|x-\theta_{2} y_{2}\right|\right) \\
e^{ \pm i 2^{N} s\left|y-\theta_{1} y_{1}\right|} \mathcal{F}^{ \pm}\left(2^{N} s\left|y-\theta_{1} y_{1}\right|\right) d s .
\end{array}
$$

Noting that for any $s \in \operatorname{supp} \varphi_{0}(s)$ and $k=0,1$, we have

$$
\left|\partial_{s}^{k} \mathcal{F}^{ \pm}\left(2^{N} s\left|x-\theta_{2} y_{2}\right|\right)\right| \lesssim 1, \quad\left|\partial_{s}^{k} \mathcal{F}^{ \pm}\left(2^{N} s\left|y-\theta_{1} y_{1}\right|\right)\right| \lesssim 1
$$

then using Lemma 2.3 with $z=\left(x, y, y_{1}, y_{2}, \theta_{1}, \theta_{2}\right)$ and

$$
\Psi(z)=\left|x-\theta_{2} y_{2}\right|+\left|y-\theta_{1} y_{1}\right|, \quad \Phi^{ \pm}\left(2^{N} s ; z\right)=\mathcal{F}^{ \pm}\left(2^{N} s\left|x-\theta_{2} y_{2}\right|\right) \cdot \mathcal{F}^{ \pm}\left(2^{N} s\left|y-\theta_{1} y_{1}\right|\right),
$$

we obtain that

$$
\sup _{x, y \in \mathbf{R}}\left|E_{1, N}^{2, \pm}\left(t ; x, y, \theta_{1}, \theta_{2}, y_{1}, y_{2}\right)\right| \lesssim 2^{N}\left(1+|t| \cdot 2^{4 N}\right)^{-\frac{1}{2}}
$$


Then, since $v(x) \lesssim(1+|x|)^{-\frac{25}{2}-}$, we have

$$
\begin{aligned}
\left|K_{1, N}^{2, \pm}(t ; x, y)\right| & \lesssim 2^{N}\left(1+|t| \cdot 2^{4 N}\right)^{-\frac{1}{2}} \int_{\mathbf{R}^{2}}\left|y_{2}^{3} v\left(y_{2}\right) \|\left[S_{2} A_{-3,1}^{2} S_{2}\right]\left(y_{2}, y_{1}\right)\right|\left|y_{1}^{3} v\left(y_{1}\right)\right| d y_{1} d y_{2} \\
& \lesssim 2^{N}\left(1+|t| \cdot 2^{N}\right)^{-\frac{1}{2}}\left\|y_{2}^{3} v\left(y_{2}\right)\right\|_{L_{y_{2}}^{2}}\left\|S_{2}-3^{2} S_{2}\right\|_{L^{2} \rightarrow L_{2}}\left\|y_{1}^{3} v\left(y_{1}\right)\right\|_{L_{y_{1}}^{2}} \\
& \lesssim 2^{N}\left(1+|t| \cdot 2^{N}\right)^{-\frac{1}{2}}
\end{aligned}
$$

uniformly in $x, y$.

2.3. High Energy Dispersive Estimates with $\alpha=0$. In this subsection, we will give the proof of the dispersive bound (2.5) with $\alpha=0$ for the large energy part. To complete the proof, we use the formula

$$
e^{-i t H} P_{a c}(H) \widetilde{\chi}(H) f(x)=\sum_{N=N_{0}+1}^{+\infty} \sum_{ \pm} \frac{ \pm 2}{\pi i} \int_{\mathbf{R}}\left(\int_{0}^{\infty} e^{-i t \mu^{4}} \varphi_{N}(\mu) \mu^{3} R_{V}^{ \pm}\left(\mu^{4}\right)(x, y) d \mu\right) f(y) d y
$$

and the following resolvent identity,

$$
R_{V}^{ \pm}\left(\mu^{4}\right)=R_{0}^{ \pm}\left(\mu^{4}\right)-R_{0}^{ \pm}\left(\mu^{4}\right) V R_{0}^{ \pm}\left(\mu^{4}\right)+R_{0}^{ \pm}\left(\mu^{4}\right) V R_{V}^{ \pm}\left(\mu^{4}\right) V R_{0}^{ \pm}\left(\mu^{4}\right)
$$

By combing Proposition 2.4 and Lemma 2.6, it suffices to establish the following bound is valid for the last two terms in (2.41).

Proposition 2.14. Assume that $|V(x)| \lesssim(1+|x|)^{-1-}$, then for any $N>N_{0}$, we have

$$
\sup _{x, y \in \mathbf{R}}\left|\int_{0}^{\infty} e^{-i t \mu^{4}} \mu^{3} \varphi_{N}(\mu)\left[R_{0}^{ \pm}\left(\mu^{4}\right) V R_{0}^{ \pm}\left(\mu^{4}\right)\right](x, y) d \mu\right| \lesssim 2^{N}\left(1+|t| \cdot 2^{4 N}\right)^{-\frac{1}{2}} .
$$

Proof. Let

$$
L_{1}^{ \pm, N}(t ; x, y):=\int_{0}^{\infty} e^{-i t \mu^{4}} \mu^{3} \varphi_{N}(\mu)\left[R_{0}^{ \pm}\left(\mu^{4}\right) V R_{0}^{ \pm}\left(\mu^{4}\right)\right](x, y) d \mu
$$

and

$$
R_{0}^{ \pm}\left(\mu^{4}\right)(x, y)=\frac{1}{4 \mu^{3}}\left( \pm i e^{ \pm i \mu|x-y|}-e^{-\mu|x-y|}\right):=\frac{e^{ \pm i \mu|x-y|}}{4 \mu^{3}} \mathcal{R}_{0}^{ \pm}(\mu|x-y|) .
$$

Let $\mu=2^{N} s$ we can rewrite $L_{1}^{ \pm, N}(x, y)$ as follows:

$$
\begin{aligned}
& L_{1}^{ \pm, N}(t ; x, y) \\
= & \frac{2^{-2 N}}{16} \int_{0}^{\infty} e^{-i t 2^{4 N} s^{4}} s^{-3} \varphi_{0}(s)\left(\int_{\mathbf{R}} e^{ \pm i 2^{N} s\left|x-y_{1}\right|} \mathcal{R}_{0}^{ \pm}\left(2^{N} s\left|x-y_{1}\right|\right) V\left(y_{1}\right) e^{ \pm i 2^{N} s\left|y-y_{1}\right|} \mathcal{R}_{0}^{ \pm}\left(2^{N} s\left|y-y_{1}\right|\right) d y_{1}\right) d s \\
= & \frac{2^{-2 N}}{16} \int_{\mathbf{R}}\left(\int_{0}^{\infty} e^{-i t 2^{4 N} s^{4}} s^{-3} \varphi_{0}(s) e^{ \pm i 2^{N} s\left|x-y_{1}\right|} e^{ \pm i 2^{N} s\left|y-y_{1}\right|} \mathcal{R}_{0}^{ \pm}\left(2^{N} s\left|x-y_{1}\right|\right) V\left(y_{1}\right) \mathcal{R}_{0}^{ \pm}\left(2^{N} s\left|y-y_{1}\right|\right) d s\right) d y_{1} .
\end{aligned}
$$

Since for any $s \in \operatorname{supp} \varphi_{0}(s)$ and $k=0,1$, we have

$$
\sup _{x, y \in \mathbf{R}}\left|\partial_{s}^{k}\left(\mathcal{R}_{0}^{ \pm}\left(2^{N} s\left|x-y_{1}\right|\right) V\left(y_{1}\right) \mathcal{R}_{0}^{ \pm}\left(2^{N} s\left|y-y_{1}\right|\right)\right)\right| \lesssim\left|V\left(y_{1}\right)\right|,
$$

then using Lemma 2.3 with $z=\left(x, y, y_{1}\right)$ and

$$
\Psi(z)=\left|x-y_{1}\right|+\left|y-y_{1}\right|, \quad \Phi\left(2^{N} s ; z\right)=\mathcal{R}_{0}^{ \pm}\left(2^{N} s\left|x-y_{1}\right|\right) \cdot \mathcal{R}_{0}^{ \pm}\left(2^{N} s\left|y-y_{1}\right|\right),
$$

we can obtain that

$$
\sup _{x, y \in \mathbf{R}}\left|L_{1}^{ \pm, N}(t ; x, y)\right| \lesssim 2^{-2 N}\left(1+|t| \cdot 2^{4 N}\right)^{-\frac{1}{2}} \int_{\mathbf{R}}\left|V\left(y_{1}\right)\right| d y_{1} \lesssim 2^{N}\left(1+|t| \cdot 2^{4 N}\right)^{-\frac{1}{2}}
$$


We next consider the last term $R_{0}^{ \pm}\left(\mu^{4}\right) V R_{V}^{ \pm}\left(\mu^{4}\right) V R_{0}^{ \pm}\left(\mu^{4}\right)$ in (2.41). To control this term, we utilize the following estimates.

Lemma 2.15. ([FSY18], Theorem 2.23) Let $k \geq 0$ and $|V(x)| \lesssim(1+|x|)^{-k-1-}$ such that $H=$ $(-\Delta)^{2}+V$ has no embedded positive eigenvalues. Then for any $\sigma>k+\frac{1}{2}, R_{V}^{ \pm}(\lambda) \in \mathcal{B}\left(L_{\sigma}^{2}(\mathbf{R}), L_{-\sigma}^{2}(\mathbf{R})\right)$ are $C^{k}$-continuous for all $\lambda>0$. Furthermore,

$$
\left\|\partial_{\lambda}^{k} R_{V}^{ \pm}(\lambda)\right\|_{L_{\sigma}^{2}(\mathbf{R}) \rightarrow L_{-\sigma}^{2}(\mathbf{R})}=O\left(|\lambda|^{-\frac{3(k+1)}{4}}\right),
$$

as $\lambda \rightarrow+\infty$.

Proposition 2.16. Assume that $|V(x)| \lesssim(1+|x|)^{-2-}$, then for any $N>N_{0}$, we have

$$
\sup _{x, y}\left|\int_{0}^{\infty} e^{-i t \mu^{4}} \mu^{3} \varphi_{N}(\mu)\left[R_{0}^{ \pm}\left(\mu^{4}\right) V R_{V}^{ \pm}\left(\mu^{4}\right) V R_{0}^{ \pm}\left(\mu^{4}\right)\right](x, y) d \mu\right| \lesssim 2^{N}\left(1+|t| \cdot 2^{4 N}\right)^{-\frac{1}{2}}
$$

Proof. For the (2.44), it suffices to prove that for any $f, g \in L^{1}(\mathbf{R})$,

$$
\left|\int_{0}^{\infty} e^{-i t \mu^{4}} \mu^{3} \varphi_{0}\left(2^{-N} \mu\right)\left\langle V R_{V}^{ \pm}\left(\mu^{4}\right) V R_{0}^{ \pm}\left(\mu^{4}\right) f,\left(R_{0}^{ \pm}\left(\mu^{4}\right)\right)^{*} g\right\rangle d \mu\right| \lesssim 2^{N}\left(1+|t| \cdot 2^{4 N}\right)^{-\frac{1}{2}}\|f\|_{L^{1}} \cdot\|g\|_{L^{1}} .
$$

Equivalently, it is enough to bound the following kernel uniformly by $O\left(2^{N}\left(1+|t| \cdot 2^{4 N}\right)^{-\frac{1}{2}}\right)$ :

$$
\widetilde{L}_{2}^{ \pm, N}(t ; x, y)=\int_{0}^{\infty} e^{-i t \mu^{4}} \mu^{3} \varphi_{0}\left(2^{-N} \mu\right)\left\langle V R_{V}^{ \pm}\left(\mu^{4}\right) V\left(R_{0}^{ \pm}\left(\mu^{4}\right)(*, y)\right)(\cdot),\left(R_{0}^{ \pm}\left(\mu^{4}\right)\right)^{*}(x, \cdot)\right\rangle d \mu
$$

where

$$
R_{0}^{ \pm}\left(\mu^{4}\right)(x, y)=\frac{1}{4 \mu^{3}}\left( \pm i e^{ \pm i \mu|x-y|}-e^{-\mu|x-y|}\right):=\frac{e^{ \pm i \mu|x-y|}}{4 \mu^{3}} \mathcal{R}_{0}^{ \pm}(\mu)(x, y) .
$$

Rewrite that

$$
\begin{aligned}
& \left\langle V R_{V}^{ \pm}\left(\mu^{4}\right) V\left(R_{0}^{ \pm}\left(\mu^{4}\right)(*, y)\right)(\cdot), R_{0}^{\mp}\left(\mu^{4}\right)(x, \cdot)\right\rangle \\
= & \frac{1}{16 \mu^{6}}\left\langle V R_{V}^{ \pm} V\left(e^{ \pm i \mu|*-y|} \mathcal{R}_{0}^{ \pm}(\mu)(*, y)\right)(\cdot),\left(e^{\mp i \mu|x-\cdot|} \mathcal{R}_{0}^{\mp}(\mu)(x, \cdot)\right)\right\rangle \\
= & \frac{1}{16 \mu^{6}} e^{ \pm i \mu|x|} e^{ \pm i \mu|y|}\left\langle V R_{V}^{ \pm} V\left(e^{ \pm i \mu(|*-y|-|y|)} \mathcal{R}_{0}^{ \pm}(\mu)(*, y)\right)(\cdot),\left(e^{\mp i \mu(|x-\cdot|-|x|)} \mathcal{R}_{0}^{\mp}(\mu)(x, \cdot)\right)\right\rangle \\
:= & \frac{1}{16 \mu^{6}} e^{ \pm i \mu(|x|+|y|)} E_{2, N}^{L, \pm}(\mu ; x, y) .
\end{aligned}
$$

Let $\mu=2^{N} s$, then we have

$$
\widetilde{L}_{2}^{ \pm, N}(t ; x, y)=\frac{2^{-2 N}}{16} \int_{0}^{\infty} e^{-i t 2^{4 N} s^{4}} e^{ \pm i 2^{N} s|x|} e^{ \pm i 2^{N} s|y|} s^{-3} \varphi_{0}(s) E_{2, N}^{L, \pm}\left(2^{N} s ; x, y\right) d s .
$$

Noting that for any $s \in \operatorname{supp} \varphi_{0}(s)$ and any $N>N_{0}$, we have

$$
\begin{aligned}
& \left|\partial_{s}^{k}\left(e^{ \pm i 2^{N} s(*-y|-| y \mid)} \mathcal{R}_{0}^{ \pm}\left(2^{N} s\right)(*, y)\right)\right| \lesssim 2^{k N}\langle *\rangle^{k}, \quad k=0,1, \\
& \left|\partial_{s}^{k}\left(e^{ \pm i 2^{N} s(|x-\cdot|-|x|)} \mathcal{R}_{0}^{ \pm}\left(2^{N} s\right)(x, \cdot)\right)\right| \lesssim 2^{k N}\langle\cdot\rangle^{k}, \quad k=0,1 .
\end{aligned}
$$

And by Lemma 2.15, for $\sigma>k+\frac{1}{2}$ with $k=0,1$, we have

$$
\left\|\partial_{s}^{k} R_{V}^{ \pm}\left(2^{4 N} s^{4}\right)\right\|_{L_{\sigma}^{2} \rightarrow L_{-\sigma}^{2}} \lesssim 2^{k N}\left(2^{N} s\right)^{-3} .
$$


Furthermore, since $|V(x)| \lesssim(1+|x|)^{-2-}$, then for $\sigma>k+\frac{1}{2}$ with $k=0,1$, we can obtain that

$$
\begin{aligned}
\left|\partial_{s}^{k} E_{2, N}^{L, \pm}\left(2^{N} s ; x, y\right)\right| & \lesssim \sum_{k=0}^{1}\left\|V(\cdot)\langle\cdot\rangle^{\sigma+1-k}\right\|_{L^{2}}^{2} \cdot\left\|\partial_{s}^{k} R_{V}^{ \pm}\left(2^{4 N} s^{4}\right)\right\|_{L_{\sigma}^{2} \rightarrow L_{-\sigma}^{2}} \\
& \lesssim 2^{k N}\left(2^{N} s\right)^{-3} .
\end{aligned}
$$

Hence, using Lemma 2.3 with $z=(x, y)$ and

$$
\Psi(z)=|x|+|y|, \quad \Phi\left(2^{N} s ; z\right)=E_{2, N}^{L, \pm}\left(2^{N} s ; x, y\right),
$$

we have

$$
\left|\widetilde{L}_{2}^{ \pm, N}(t ; x, y)\right| \lesssim 2^{(k-5) N}\left(1+|t| \cdot 2^{4 N}\right)^{-\frac{1}{2}} \lesssim 2^{N}\left(1+|t| \cdot 2^{4 N}\right)^{-\frac{1}{2}}
$$

uniform in $x, y$ for any $N>N_{0}$.

2.4. Proofs of the decay estimates with regular term $H^{\frac{\alpha}{4}}$ for $0<\alpha<1$. In the final subsection, we will give the proof of the estimates (2.4) and (2.6). By using functional calculus and the Stone's formula, we have

$$
\begin{aligned}
H^{\frac{\alpha}{4}} e^{-i t H} P_{a c}(H) f(x) & =\frac{2}{\pi i} \int_{0}^{\infty} e^{-i t \lambda} \lambda^{\frac{\alpha}{4}}\left[R_{V}^{+}(\lambda)-R_{V}^{-}(\lambda)\right] f d \lambda \\
& =\int_{\mathbf{R}}\left(\frac{2}{\pi i} \int_{0}^{\infty} e^{-i t \mu^{4}} \mu^{3+\alpha}\left[R_{V}^{+}\left(\mu^{4}\right)-R_{V}^{-}\left(\mu^{4}\right)\right](x, y) d \mu\right) f(y) d y .
\end{aligned}
$$

Similar to the proof of Theorem 1.3 for $\alpha=0$, by using dyadic partition of unity, we can obtain for any $N \in \mathbf{Z}$ and $\alpha \geq 0$,

$$
\sup _{x, y \in \mathbf{R}}\left|\int_{0}^{\infty} e^{-i t \mu^{4}} \mu^{3+\alpha} \varphi_{N}(\mu)\left[R_{V}^{+}\left(\mu^{4}\right)-R_{V}^{-}\left(\mu^{4}\right)\right](x, y) d \mu\right| \lesssim 2^{(\alpha+1) N}\left(1+|t| \cdot 2^{4 N}\right)^{-\frac{1}{2}} .
$$

Then, by Lemma 2.6, for any $0<\alpha<1$, we have

$$
\begin{aligned}
& \sup _{x, y \in \mathbf{R}}\left|\int_{0}^{\infty} e^{-i t \mu^{4}} \mu^{3+\alpha}\left[R_{V}^{+}\left(\mu^{4}\right)-R_{V}^{-}\left(\mu^{4}\right)\right](x, y) d \mu\right| \\
\lesssim & \sum_{N=-\infty}^{+\infty} \sup _{x, y \in \mathbf{R}}\left|\int_{0}^{\infty} e^{-i t \mu^{4}} \varphi_{N}(\mu) \mu^{3+\alpha}\left[R_{V}^{+}\left(\mu^{4}\right)-R_{V}^{-}\left(\mu^{4}\right)\right](x, y) d \mu\right| \lesssim|t|^{-\frac{1+\alpha}{4}} .
\end{aligned}
$$

Hence, for any $0<\alpha<1$, we have

$$
\left\|H^{\frac{\alpha}{4}} e^{-i t H} P_{a c}(H)\right\|_{L^{1}(\mathbf{R}) \rightarrow L^{\infty}(\mathbf{R})} \lesssim|t|^{-\frac{1+\alpha}{4}}, t \neq 0 .
$$

\section{Proof of Theorem 1.8}

In this section, we show the processes how to derive the asymptotic expansions of $\left(M^{ \pm}(\mu)\right)^{-1}$. Recall that the integral kernel of $R_{0}^{ \pm}\left(\mu^{4}\right)$ are given by

$$
R_{0}^{ \pm}\left(\mu^{4}\right)(x, y)=\frac{1}{4 \mu^{3}}\left( \pm i e^{ \pm i \mu|x-y|}-e^{-\mu|x-y|}\right)
$$

When $\mu|x-y|<1$, we have the following representation for the $R_{0}^{ \pm}\left(\mu^{4}\right)(x, y)$ :

$$
\begin{aligned}
R_{0}^{ \pm}\left(\mu^{4}\right)(x, y)= & \frac{a^{ \pm}}{\mu^{3}} I(x, y)+\frac{a_{-1}^{ \pm}}{\mu} G_{-1}(x, y)+G_{0}(x, y)+a_{1}^{ \pm} \mu G_{1}(x, y)+a_{3}^{ \pm} \mu^{3} G_{3}(x, y) \\
& +\mu^{4} G_{4}(x, y)+\sum_{k=5}^{N} a_{k}^{ \pm} \mu^{k} G_{k}(x, y)+O\left(\mu^{N+1}|x-y|^{N+4}\right) .
\end{aligned}
$$


Here $G_{k}(x, y)$ are integral kernels which defined as follows:

$$
\begin{aligned}
G_{-1}(x, y) & =|x-y|^{2}, \quad G_{0}(x, y)=\frac{|x-y|^{3}}{12}, \quad G_{1}(x, y)=|x-y|^{4}, \\
G_{3}(x, y) & =|x-y|^{6}, \quad G_{4}(x, y)=\frac{|x-y|^{7}}{2 \times(7 !)}, \quad G_{k}(x, y)=|x-y|^{3+k}(k \geq 5),
\end{aligned}
$$

and the coefficients $a^{ \pm}=\frac{-1 \pm i}{4}, a_{k}^{ \pm}=\frac{( \pm i)^{k+4}+(-1)^{k+4}}{4 \times(k+3) !}$. Notice that, $G_{0}(x)=\frac{|x|^{3}}{12}$ is the fundamental solution of $\Delta^{2}$ in $\mathbf{R}$. In fact, when $\mu|x-y| \gtrsim 1$, the expansions remains valid. It is obvious that the first two terms in (3.1) are singular, which is different from at most one singular term on $\mu$ for $n \geq 3$, see e.g. [EGT21, FWY18, GT19].

For convenience, we introduce two notations here. For operators $\varepsilon(\mu)$ with some parameter $\mu$, we write $\left.\varepsilon(\mu)=O_{1}\left(\mu^{-\alpha} g(x, y)\right)\right)$ if it's kernel $\varepsilon(\mu)(x, y)$ has the property

$$
\mu^{\alpha}|\varepsilon(\mu)(x, y)|+\mu^{\alpha+1}\left|\partial_{\mu} \varepsilon(\mu)(x, y)\right| \lesssim g(x, y) .
$$

And we denote $\Gamma_{k}(\mu)$ as a $\mu$ dependent operator which satisfies

$$
\left\|\left|\Gamma_{k}(\mu)\right|\right\|_{L^{2} \rightarrow L^{2}}+\mu\left\|\partial_{\mu}\left|\Gamma_{k}(\mu)\right|\right\|_{L^{2} \rightarrow L^{2}} \lesssim \mu^{k}, \quad \mu>0,
$$

Noting that the operator $\Gamma_{k}(\mu)$ will vary from line to line.

Let $T_{0}:=U+v G_{0} v$ and $P=\|V\|_{L^{1}(\mathbf{R})}^{-1} v\langle v, \cdot\rangle$ denote the orthogonal projection onto the span of $v$. Since $M^{ \pm}(\mu)=U+v R_{0}^{ \pm}\left(\mu^{4}\right) v$ ( see Theorem 1.8), then we obtain the following expansions of $M^{ \pm}(\mu)$.

Lemma 3.1. Let $\widetilde{a}^{ \pm}=a^{ \pm}\|V\|_{L^{1}(\mathbf{R})}$. Assume that $|V(x)| \lesssim(1+|x|)^{-\beta}$ with some $\beta>0$, the following expansions for $M^{ \pm}(\mu)$ in $L^{2}(\mathbf{R})$ hold:

(i) If $\beta>13$, then we have

$$
M^{ \pm}(\mu)=\frac{\widetilde{a}^{ \pm}}{\mu^{3}} P+\frac{a_{-1}^{ \pm}}{\mu} v G_{-1} v+T_{0}+a_{1}^{ \pm} \mu v G_{1} v+\Gamma_{3}(\mu) .
$$

(ii) If $\beta>17$, then we have

$$
M^{ \pm}(\mu)=\frac{\widetilde{a}^{ \pm}}{\mu^{3}} P+\frac{a_{-1}^{ \pm}}{\mu} v G_{-1} v+T_{0}+a_{1}^{ \pm} \mu v G_{1} v+a_{3}^{ \pm} \mu^{3} v G_{3} v+\mu^{4} v G_{4} v+\Gamma_{5}(\mu) .
$$

(iii) If $\beta>25$, we have

$$
\begin{aligned}
M^{ \pm}(\mu)= & \frac{\widetilde{a}^{ \pm}}{\mu^{3}} P+\frac{a_{-1}^{ \pm}}{\mu} v G_{-1} v+T_{0}+a_{1}^{ \pm} \mu v G_{1} v+a_{3}^{ \pm} \mu^{3} v G_{3} v+\mu^{4} v G_{4} v \\
& +\sum_{k=5}^{8} a_{k}^{ \pm} \mu^{k} v G_{k} v+\Gamma_{9}(\mu) .
\end{aligned}
$$

Proof. We only give a proof of (3.5), the other cases are similar. If $\mu|x-y|<1$, using (3.1), we have

$$
R_{0}^{ \pm}\left(\mu^{4}\right)(x, y)=\frac{a^{ \pm}}{\mu^{3}}+\frac{a_{-1}^{ \pm}}{\mu} G_{-1}+G_{0}+a_{1}^{ \pm} \mu G_{1}+O_{1}\left(\mu^{3}|x-y|^{6}\right)
$$


If $\mu|x-y|>1$, using (1.19), we have

$$
\begin{aligned}
& R_{0}^{ \pm}\left(\mu^{4}\right)(x, y) \\
= & \frac{a^{ \pm}}{\mu^{3}}+\frac{a_{-1}^{ \pm}}{\mu} G_{-1}+G_{0}+a_{1}^{ \pm} \mu G_{1}+\left(\frac{ \pm i e^{ \pm i \mu|x-y|}-e^{-\mu|x-y|}}{4 \mu^{3}}-\frac{a^{ \pm}}{\mu^{3}}-\frac{a_{-1}^{ \pm}}{\mu} G_{-1}-G_{0}-a_{1}^{ \pm} \mu G_{1}\right) \\
= & \frac{a^{ \pm}}{\mu^{3}}+\frac{a_{-1}^{ \pm}}{\mu} G_{-1}+G_{0}+a_{1}^{ \pm} \mu G_{1}+O_{1}\left(\mu^{3}|x-y|^{6}\right) .
\end{aligned}
$$

Using (3.8) and (3.9) in (3.5), we have

$$
\left|\left(M^{ \pm}(\mu)-\frac{\widetilde{a}^{ \pm}}{\mu^{3}} P-\frac{a_{-1}^{ \pm}}{\mu} v G_{-1} v-T_{0}-a_{1}^{ \pm} \mu v G_{1} v\right)\right| \lesssim v(x) v(y)|x-y|^{6} \mu^{3}
$$

and

$$
\left|\partial_{\mu}\left(M^{ \pm}(\mu)-\frac{\widetilde{a}^{ \pm}}{\mu^{3}} P-\frac{a_{-1}^{ \pm}}{\mu} v G_{-1} v-T_{0}-a_{1}^{ \pm} \mu v G_{1} v\right)\right| \lesssim v(x) v(y)|x-y|^{6} \mu^{2}
$$

Since the error term is a Hilbert-Schmidt operator if $v(x) \lesssim(1+|x|)^{-\frac{13}{2}-}$, this implies (3.5) .

Remark 3.2. If we just want to get a weak expansions of $\left(M^{ \pm}(\mu)\right)^{-1}$ (see Remark 1.10 of Introduction), then we only need the following expansions of $M^{ \pm}(\mu)$ in the following Table 2.

\begin{tabular}{|c|c|}
\hline$\beta>7+2 l$ & $M^{ \pm}(\mu)=\frac{\widetilde{a}^{ \pm}}{\mu^{3}} P+\frac{a_{-1}^{ \pm}}{\mu} v G_{-1} v+T_{0}+\Gamma_{l}(\mu), l \in[0,1]$ \\
\hline$\beta>9+2 l$ & $M^{ \pm}(\mu)=\frac{\widetilde{a}^{ \pm}}{\mu^{3}} P+\frac{a_{-1}^{ \pm}}{\mu} v G_{-1} v+T_{0}+a_{1}^{ \pm} \mu v G_{1} v+\Gamma_{1+l}(\mu), l \in[0,2]$ \\
\hline$\beta>13+2 l$ & $M^{ \pm}(\mu)=\frac{\widetilde{a}^{ \pm}}{\mu^{3}} P+\frac{a_{-1}^{ \pm}}{\mu} v G_{-1} v+T_{0}+a_{1}^{ \pm} \mu v G_{1} v+a_{3}^{ \pm} \mu^{3} v G_{3} v+\Gamma_{3+l}(\mu), l \in[0,1]$ \\
\hline
\end{tabular}

TABLE 2. $M^{ \pm}(\mu)$ expansions

Now, we give the following lemma used repeatedly to determine the inverse of $\left(M^{ \pm}(\mu)\right)^{-1}$.

Lemma 3.3. ([JN01, Lemma 2.1]) Let $A$ be a closed operator and $S$ be a projection. Suppose $A+S$ has a bounded inverse. Then $A$ has a bounded inverse if and only if

$$
B:=S-S(A+S)^{-1} S
$$

has a bounded inverse in $S \mathcal{H}$, and in this case

$$
A^{-1}=(A+S)^{-1}+(A+S)^{-1} S B^{-1} S(A+S)^{-1} .
$$

Recall from (3.5), let $M^{ \pm}(\mu)=\frac{\widetilde{a}^{ \pm}}{\mu^{3}} \widetilde{M^{ \pm}}(\mu)$, we have

$$
\begin{aligned}
\widetilde{M^{ \pm}}(\mu)= & P+\frac{a_{-1}^{ \pm}}{\widetilde{a}^{ \pm}} \mu^{2} v G_{-1} v+\frac{\mu^{3}}{\widetilde{a}^{ \pm}} T_{0}+\frac{a_{1}^{ \pm}}{\widetilde{a}^{ \pm}} \mu^{4} v G_{1} v+\frac{a_{3}^{ \pm}}{\widetilde{a}^{ \pm}} \mu^{6} v G_{3} v+\frac{\mu^{7}}{\widetilde{a}^{ \pm}} v G_{4} v \\
& +\sum_{k=5}^{10} \frac{a_{k}^{ \pm}}{\widetilde{a}^{ \pm}} \mu^{k+3} v G_{k} v+\Gamma_{14}(\mu) .
\end{aligned}
$$


Thus it suffices to derive the asymptotic expansion of $\left(\widetilde{M^{ \pm}}(\mu)\right)^{-1}$ as $\mu \rightarrow 0$. Denote $Q=I-P$, it is obvious that

$$
\begin{aligned}
\widetilde{M^{ \pm}}(\mu)+Q= & I+\frac{a_{-1}^{ \pm}}{\widetilde{a}^{ \pm}} \mu^{2} v G_{-1} v+\frac{\mu^{3}}{\widetilde{a}^{ \pm}} T_{0}+\frac{a_{1}^{ \pm}}{\widetilde{a}^{ \pm}} \mu^{4} v G_{1} v+\frac{a_{3}^{ \pm}}{\widetilde{a}^{ \pm}} \mu^{6} v G_{3} v+\frac{\mu^{7}}{\widetilde{a}^{ \pm}} v G_{4} v \\
& +\sum_{k=5}^{10} \frac{a_{k}^{ \pm}}{\widetilde{a}^{ \pm}} \mu^{k+3} v G_{k} v+\Gamma_{14}(\mu) .
\end{aligned}
$$

Since $v G_{-1} v$ is a bounded operator from $L^{2}(\mathbf{R})$ to $L^{2}(\mathbf{R})$, then using Neumann series, we can obtain that

$$
\left(\widetilde{M^{ \pm}}(\mu)+Q\right)^{-1}=I-\sum_{k=1}^{12} \mu^{k+1} B_{k}^{ \pm}+\Gamma_{14}(\mu),
$$

where $B_{k}^{ \pm}(1 \leq k \leq 12)$ are bounded operators in $L^{2}(\mathbf{R})$ and it is easy to know that

$$
\begin{aligned}
& B_{1}^{ \pm}=\frac{a_{-1}^{ \pm}}{\widetilde{a}^{ \pm}} v G_{-1} v, \quad B_{2}^{ \pm}=\frac{1}{\widetilde{a}^{ \pm}} T_{0}, \quad B_{3}^{ \pm}=\frac{a_{1}^{ \pm}}{\widetilde{a}^{ \pm}} v G_{1} v-\frac{\left(a_{-1}^{ \pm}\right)^{2}}{\left(\widetilde{a}^{ \pm}\right)^{2}}\left(v G_{-1} v\right)^{2}, \\
& B_{4}^{ \pm}=-\frac{a_{-1}^{ \pm}}{\left(\widetilde{a}^{ \pm}\right)^{2}}\left(v G_{-1} v T_{0}+T_{0} v G_{-1} v\right), \\
& B_{5}^{ \pm}=\frac{a_{3}^{ \pm}}{\widetilde{a}^{ \pm}} v G_{3} v+\frac{\left(a_{-1}^{ \pm}\right)^{3}}{\left(\widetilde{a}^{ \pm}\right)^{3}}\left(v G_{-1} v\right)^{3}-\frac{1}{\left(\widetilde{a}^{ \pm}\right)^{2}} T_{0}^{2}-\frac{a_{-1}^{ \pm} a_{1}^{ \pm}}{\left(\widetilde{a}^{ \pm}\right)^{2}}\left(v G_{-1} v \cdot v G_{1} v+v G_{1} v \cdot v G_{-1} v\right), \\
& B_{6}^{ \pm}=\frac{1}{\widetilde{a}^{ \pm}} v G_{4} v+\frac{\left(a_{-1}^{ \pm}\right)^{2}}{\left(\widetilde{a}^{ \pm}\right)^{3}}\left(\left(v G_{-1} v\right)^{2} T_{0}+v G_{-1} v T_{0} v G_{-1} v+T_{0}\left(v G_{-1} v\right)^{2}\right)-\frac{a_{1}^{ \pm}}{\left(\widetilde{a}^{ \pm}\right)^{2}}\left(v G_{1} v T_{0}+T_{0} v G_{1} v\right) .
\end{aligned}
$$

For the convenience, we denote some inverse operators as follows:

$$
\begin{array}{lll}
D_{0}=\left(Q v G_{-1} v Q+S_{0}\right)^{-1} \text { on } Q L^{2}(\mathbf{R}) ; & D_{1}=\left(S_{0} T_{0} S_{0}+S_{1}\right)^{-1} \text { on } S_{0} L^{2}(\mathbf{R}) ; \\
D_{2}=\left(T_{1}+S_{2}\right)^{-1} \text { on } S_{1} L^{2}(\mathbf{R}) ; & D_{3}=\left(T_{2}+S_{3}\right)^{-1} \text { on } S_{2} L^{2}(\mathbf{R}) .
\end{array}
$$

Next, we list the orthogonality relations of various operators and projections which we need in the proof of Theorem 1.8 .

$$
\begin{aligned}
& Q D_{0}=D_{0} Q=D_{0} ; \\
& S_{i} D_{j}=D_{j} S_{i}=D_{j}, i<j ; \quad S_{i} D_{j}=D_{j} S_{i}=S_{i}, i \geq j ; \quad D_{i} D_{j}=D_{j} D_{i}=D_{j}, i<j ; \\
& Q B_{1}^{ \pm} S_{0}=S_{0} B_{1}^{ \pm} Q=S_{2} B_{1}^{ \pm}=B_{1}^{ \pm} S_{2}=S_{3} B_{1}^{ \pm}=B_{1}^{ \pm} S_{3}=0 ; \\
& S_{0} B_{2}^{ \pm} S_{1}=S_{1} B_{2}^{ \pm} S_{0}=Q B_{2}^{ \pm} S_{2}=S_{2} B_{2}^{ \pm} Q=S_{3} B_{2}^{ \pm}=B_{2}^{ \pm} S_{3}=0 ; \\
& S_{0} B_{3}^{ \pm} S_{2}=S_{2} B_{3}^{ \pm} S_{0}=Q B_{3}^{ \pm} S_{3}=S_{3} B_{3}^{ \pm} Q=0 ; \\
& S_{3} B_{4}^{ \pm}=B_{4}^{ \pm} S_{3}=S_{2} B_{5}^{ \pm} S_{3}=S_{3} B_{5}^{ \pm} S_{2}=0 .
\end{aligned}
$$

Now, we turn to the proof of Theorem 1.8. The following lemma which will be used in the proof is an important classification of the projection spaces $S_{j} L^{2}(\mathbf{R})(j=0,1,2,3)$.

Lemma 3.4. We have

(1) $f \in S_{0} L^{2}(\mathbf{R})$ if and only if $f \in \operatorname{ker} Q v G_{-1} v Q$ on $Q L^{2}(\mathbf{R})$;

(2) $f \in S_{1} L^{2}(\mathbf{R})$ if and only if $f \in \operatorname{ker} S_{0} T_{0} S_{0}$ on $S_{0} L^{2}(\mathbf{R})$;

(3) $f \in S_{2} L^{2}(\mathbf{R})$ if and only if $f \in \operatorname{ker} T_{1}$ on $S_{1} L^{2}(\mathbf{R})$ with

$$
T_{1}:=S_{1} v G_{1} v S_{1}+\frac{6}{\|V\|_{L^{1}}} S_{1} v G_{-1} v P v G_{-1} v S_{1}-384 S_{1} T_{0} D_{0} T_{0} S_{1} .
$$


(4) $f \in S_{3} L^{2}(\mathbf{R})$ if and only if $f \in \operatorname{ker} T_{2}$ on $S_{2} L^{2}(\mathbf{R})$ with

$$
\begin{aligned}
T_{2}:= & S_{2} v G_{3} v S_{2}-\frac{8 \cdot(6 !)}{\|V\|_{L^{1}}} S_{2} T_{0}^{2} S_{2}+\frac{5}{2} S_{2} v G_{1} v D_{0} v G_{1} v S_{2}+\frac{48 \cdot(6 !)}{\|V\|_{L^{1}}^{2}}\left(S_{2} T_{0} v G_{-1} v\right. \\
& \left.-\frac{\|V\|_{L^{1}}}{6} S_{2} v G_{1} v D_{0} T_{0}\right) D_{2}\left(v G_{-1} v T_{0} S_{2}-\frac{\|V\|_{L^{1}}}{6} T_{0} D_{0} v G_{1} v S_{2}\right) .
\end{aligned}
$$

Proof. (1) Let $f \in Q L^{2}(\mathbf{R})$ and $f \in \operatorname{ker} Q v G_{-1} v Q$, that is, $Q v G_{-1} v Q f=0$. Then we have

$$
0=\left\langle Q v G_{-1} v Q f, f\right\rangle=\left\langle v G_{-1} v Q f, Q f\right\rangle=\left\langle v G_{-1} v f, f\right\rangle .
$$

Notice that

$$
G_{-1} v f(x)=\int_{\mathbf{R}}|x-y|^{2} v(y) f(y) d y,
$$

and $Q(v)=0$, i.e. $\langle f, v\rangle=\int_{\mathbf{R}} v(x) f(x) d x=0$. So we have

$$
\begin{aligned}
0=\left\langle v G_{-1} v f, f\right\rangle & =\int_{\mathbf{R}}\left(v G_{-1} v f\right)(x) \overline{f(x)} d x \\
& =\int_{\mathbf{R}} \overline{v(x) f(x)}\left(\int_{\mathbf{R}}|x-y|^{2} v(y) f(y) d y\right) d x \\
& =\int_{\mathbf{R}} \overline{v(x) f(x)}\left(\int_{\mathbf{R}}\left(x^{2}-2 x y+y^{2}\right) v(y) f(y) d y\right) d x \\
& =-2\left|\int_{\mathbf{R}} x v(x) f(x) d x\right|^{2}=-2|\langle f, x v\rangle|^{2},
\end{aligned}
$$

which implies that $\langle f, x v\rangle=0$. Hence $f \in S_{0} L^{2}(\mathbf{R})$.

On the other hand, if $f \in S_{0} L^{2}(\mathbf{R})$, that is,

$$
\langle f, v\rangle=\int_{\mathbf{R}} v(x) f(x) d x=0, \quad\langle f, x v\rangle=\int_{\mathbf{R}} x v(x) f(x) d x=0 .
$$

Since $S_{0} L^{2}(\mathbf{R}) \subseteq Q L^{2}(\mathbf{R})$, thus we have

$$
Q v G_{-1} v Q f=Q v(x) \int_{\mathbf{R}}\left(x^{2}-2 x y+y^{2}\right) v(y) f(y) d y=\left\langle f, x^{2} v\right\rangle Q(v(x))=0,
$$

which means that $f \in \operatorname{ker} Q v G_{-1} v Q$.

(2) It is obvious by the definition of $S_{1}$, see Table 1 .

(3) First, we prove the Claim: Denote $v_{1}:=Q(x v(x))=x v(x)-\frac{\langle v, x v\rangle}{\|V\|_{L^{1}}} v(x)$, then we have

$$
\left\langle D_{0} v_{1}, v_{1}\right\rangle=-\frac{1}{2}
$$

In fact, using $Q(v(x))=0, S_{0}(v(x))=S_{0}(x v(x))=0$ and $\left\langle v, v_{1}\right\rangle=0$, we have

$$
\begin{aligned}
\left(Q v G_{-1} v Q+S_{0}\right) v_{1} & =Q v G_{-1} v Q v_{1} \\
& =Q v(x) \int_{\mathbf{R}}\left(x^{2}-2 x y+y^{2}\right) v(y) v_{1}(y) d y \\
& =-2 Q(x v(x)) \int_{\mathbf{R}} y v(y) v_{1}(y) d y \\
& =-2 v_{1}\left\langle v_{1}, v_{1}\right\rangle .
\end{aligned}
$$

Hence, we obtain that

$$
D_{0} v_{1}=\left(Q v G_{-1} v Q+S_{0}\right)^{-1} v_{1}=-\frac{v_{1}}{2\left\langle v_{1}, v_{1}\right\rangle}
$$


furthermore, we have $\left\langle D_{0} v_{1}, v_{1}\right\rangle=-\frac{1}{2}$.

Let $f \in S_{1} L^{2}(\mathbf{R})$ and $f \in \operatorname{ker} T_{1}$. Recall (3.22), we have

$$
\begin{aligned}
0=\left\langle T_{1} f, f\right\rangle= & \left\langle S_{1} v G_{1} v S_{1} f, f\right\rangle+\frac{6}{\|V\|_{L^{1}}}\left\langle S_{1} v G_{-1} v P v G_{-1} v S_{1} f, f\right\rangle \\
& -384\left\langle S_{1} T_{0} D_{0} T_{0} S_{1} f, f\right\rangle .
\end{aligned}
$$

Firstly, for the first part of (3.26), using $S_{1}(v(x))=S_{1}(x v(x))=0$, we have

$$
\begin{aligned}
S_{1} v G_{1} v S_{1} f & =S_{1} v(x) \int_{\mathbf{R}}\left(x^{4}-4 x^{3} y-4 x y^{3}+6 x^{2} y^{2}+y^{4}\right) v(y) f(y) d y \\
& =6\left\langle f, x^{2} v\right\rangle S_{1}\left(x^{2} v\right) .
\end{aligned}
$$

Thus, we have

$$
\left\langle S_{1} v G_{1} v S_{1} f, f\right\rangle=6\left|\left\langle f, x^{2} v\right\rangle\right|^{2} .
$$

Next, for the second part of (3.26), since $P f=\frac{\langle f, v\rangle}{\|V\|_{L^{1}}} v$ and $S_{1}(v(x))=S_{1}(x v(x))=0$, we have

$$
P v G_{-1} v S_{1} f=P v(x) \int_{\mathbf{R}^{2}}|x-y|^{2} v(y) f(y) d y=v(x) \int_{\mathbf{R}} x^{2} v(x) f(x) d x=\left\langle f, x^{2} v\right\rangle v(x),
$$

and

$$
S_{1} v G_{-1} v P v G_{-1} v S_{1} f=\left\langle f, x^{2} v\right\rangle\|V\|_{L^{1}} S_{1}\left(x^{2} v(x)\right) .
$$

Thus

$$
\left\langle S_{1} v G_{-1} v P v G_{-1} v S_{1} f, f\right\rangle=\|V\|_{L^{1}} \cdot\left|\left\langle f, x^{2} v\right\rangle\right|^{2} .
$$

Finally, for the third part of (3.26), using $Q D_{0}=D_{0} Q=D_{0}$ and by the definition of $v_{1}$, we know that $v_{1} \in\left(Q-S_{0}\right) L^{2}(\mathbf{R})$. Indeed, we have $\left(Q-S_{0}\right) L^{2}(\mathbf{R})=\operatorname{span}\left\{v_{1}\right\}$, then we can obtain that

$$
\left(Q-S_{0}\right) T_{0} f=\frac{\left\langle T_{0} f, v_{1}\right\rangle}{\left\langle v_{1}, v_{1}\right\rangle}
$$

Thus, using (3.25) and $D_{0} P=P D_{0}=0, S_{1} T_{0} S_{0}=S_{0} T_{0} S_{1}=0$, for all $f \in S_{1} L^{2}(\mathbf{R})$, we have

$$
\begin{aligned}
S_{1} T_{0} D_{0} T_{0} S_{1} f & =S_{1} T_{0}\left[P+\left(Q-S_{0}\right)+S_{0}\right] D_{0}\left[P+\left(Q-S_{0}\right)+S_{0}\right] T_{0} f \\
& =S_{1} T_{0}\left(Q-S_{0}\right) D_{0}\left(Q-S_{0}\right) T_{0} f \\
& =-\frac{\left\langle T_{0} f, v_{1}\right\rangle}{2\left\langle v_{1}, v_{1}\right\rangle^{2}} S_{1}\left(T_{0} v_{1}\right) .
\end{aligned}
$$

Thus,

$$
\left\langle S_{1} T_{0} D_{0} T_{0} S_{1} f, f\right\rangle=-\frac{\left|\left\langle T_{0} f, v_{1}\right\rangle\right|^{2}}{2\left\langle v_{1}, v_{1}\right\rangle^{2}} .
$$

Hence, substituting (3.27), (3.28) and (3.29) into (3.26), we have

which implies that

$$
0=\left\langle T_{1} f, f\right\rangle=12\left|\left\langle f, x^{2} v\right\rangle\right|^{2}+192 \frac{\left|\left\langle T_{0} f, v_{1}\right\rangle\right|^{2}}{\left\langle v_{1}, v_{1}\right\rangle^{2}},
$$

$$
\left\langle f, x^{2} v\right\rangle=0, \quad\left\langle T_{0} f, v_{1}\right\rangle=0 .
$$

Furthermore, since $f \in S_{1} L^{2}(\mathbf{R})$, thus $S_{0} T_{0} f=0$, combined with $\left\langle T_{0} f, v_{1}\right\rangle=0$, we have $Q T_{0} f=0$. Hence we have $f \in S_{2} L^{2}(\mathbf{R})$.

On the other hand, if $f \in S_{2} L^{2}(\mathbf{R})$, then it is easy to know that

$$
T_{1} f=12\left\langle f, x^{2} v\right\rangle S_{1}\left(x^{2} v\right)+192 \frac{\left\langle T_{0} f, v_{1}\right\rangle}{\left\langle v_{1}, v_{1}\right\rangle^{2}} S_{1}\left(T_{0} v_{1}\right)=0,
$$


which means that $f \in \operatorname{ker} T_{1}$.

(4) Let $f \in S_{2} L^{2}(\mathbf{R})$ and $f \in \operatorname{ker} T_{2}$. It follows that from (3.23)

$$
\begin{aligned}
0= & \left\langle T_{2} f, f\right\rangle=\left\langle S_{2} v G_{3} v S_{2} f, f\right\rangle-\frac{8 \cdot(6 !)}{\|V\|_{L^{1}}}\left\langle S_{2} T_{0}^{2} S_{2} f, f\right\rangle+\frac{5}{2}\left\langle S_{2} v G_{1} v D_{0} v G_{1} v S_{2} f, f\right\rangle \\
& +\frac{48 \cdot(6 !)}{\|V\|_{L^{1}}^{2}}\left\langle\left(S_{2} T_{0} v G_{-1} v-\frac{\|V\|_{L^{1}}}{6} S_{2} v G_{1} v D_{0} T_{0}\right) D_{2}\left(v G_{-1} v T_{0} S_{2}-\frac{\|V\|_{L^{1}}}{6} T_{0} D_{0} v G_{1} v S_{2}\right) f, f\right\rangle .
\end{aligned}
$$

For the first part of (3.30), using $S_{2}(v(x))=S_{2}(x v(x))=S_{2}\left(x^{2} v(x)\right)=0$ and $Q T_{0} f=0$, we have

$$
S_{2} v G_{3} v S_{2} f=S_{2} v(x) \int_{\mathbf{R}}|x-y|^{6} v(y) f(y) d y=-20\left\langle f, x^{3} v\right\rangle S_{2}\left(x^{3} v(x)\right),
$$

thus

$$
\left\langle S_{2} v G_{3} v f, f\right\rangle=-20\left|\left\langle f, x^{3} v\right\rangle\right|^{2} .
$$

Secondly, for the second part of (3.30), using $Q T_{0} f=0$, we have

so

$$
S_{2} T_{0}^{2} S_{2} f=S_{2} T_{0} P T_{0} S_{2} f=\frac{\left\langle T_{0} f, v\right\rangle}{\|V\|_{L^{1}}} S_{2}\left(T_{0} v\right),
$$

$$
\left\langle S_{2} T_{0}^{2} S_{2} f, f\right\rangle=\frac{\left\langle T_{0} f, v\right\rangle^{2}}{\|V\|_{L^{1}}} .
$$

Then, for the third part of (3.30), using (3.25) and

$$
Q D_{0} Q=D_{0}, \quad Q(x v(x))=v_{1}, \quad S_{2}(v(x))=S_{2}(x v(x))=S_{2}\left(x^{2} v(x)\right)=0,
$$

we have

$$
S_{2} v G_{1} v D_{0} v G_{1} v S_{2} f=S_{2} v G_{1} v Q D_{0} Q v G_{1} v f=-8\left\langle f, x^{3} v\right\rangle S_{2}\left(x^{3} v\right),
$$

and

$$
\left\langle S_{2} v G_{1} v D_{0} v G_{1} v S_{2} f, f\right\rangle=-8\left|\left\langle f, x^{3} v\right\rangle\right|^{2} .
$$

Finally, for the last part of (3.30), using (3.25) and

$$
S_{1} D_{2} S_{1}=D_{2}, \quad S_{1}(v)=S_{1}(x v)=0, \quad S_{2}(v(x))=S_{2}(x v(x))=S_{2}\left(x^{2} v(x)\right)=0,
$$

we have

$$
\begin{gathered}
S_{2} T_{0} v G_{-1} v D_{2} v G_{-1} v T_{0} S_{2} f=\left\langle T_{0} f, v\right\rangle \cdot\left\langle D_{2} S_{1}\left(x^{2} v\right), S_{1}\left(x^{2} v\right)\right\rangle S_{2}\left(T_{0} v\right), \\
S_{2} T_{0} v G_{-1} v D_{2} T_{0} D_{0} v G_{1} v S_{2} f=2 \frac{\left\langle f, x^{3} v\right\rangle}{\left\langle v_{1}, v_{1}\right\rangle} \cdot\left\langle D_{2} S_{1}\left(T_{0} v_{1}\right), S_{1}\left(x^{2} v\right)\right\rangle S_{2}\left(T_{0} v\right), \\
S_{2} v G_{1} v D_{0} T_{0} D_{2} v G_{-1} v T_{0} S_{2} f=2 \frac{\left\langle T_{0} f, v\right\rangle}{\left\langle v_{1}, v_{1}\right\rangle} \cdot\left\langle D_{2} S_{1}\left(T_{0} v_{1}\right), S_{1}\left(x^{2} v\right)\right\rangle S_{2}\left(x^{3} v\right), \\
S_{2} v G_{1} v D_{0} T_{0} D_{2} T_{0} D_{0} v G_{1} v S_{2} f=4 \frac{\left\langle f, x^{3} v\right\rangle}{\left\langle v_{1}, v_{1}\right\rangle^{2}} \cdot\left\langle D_{2} S_{1}\left(T_{0} v_{1}\right), S_{1}\left(T_{0} v_{1}\right)\right\rangle S_{2}\left(x^{3} v\right) .
\end{gathered}
$$

Denote $X=\left\langle f, x^{3} v\right\rangle, Y=\left\langle T_{0} f, v\right\rangle$, we obtain that

$$
\begin{aligned}
& \left\langle\left(S_{2} T_{0} v G_{-1} v-\frac{\|V\|_{L^{1}}}{6} S_{2} v G_{1} v D_{0} T_{0}\right) D_{2}\left(v G_{-1} v T_{0} S_{2}-\frac{\|V\|_{L^{1}}}{6} T_{0} D_{0} v G_{1} v S_{2}\right) f, f\right\rangle \\
= & \frac{\|V\|_{L^{1}}^{2} \cdot\left\langle D_{2} S_{1}\left(T_{0} v_{1}\right), S_{1}\left(T_{0} v_{1}\right)\right\rangle}{9\left\langle v_{1}, v_{1}\right\rangle^{2}} \cdot|X|^{2}+\left\langle D_{2} S_{1}\left(x^{2} v\right), S_{1}\left(x^{2} v\right)\right\rangle \cdot|Y|^{2} \\
& -\frac{2\|V\|_{L^{1}} \cdot\left\langle D_{2} S_{1}\left(T_{0} v_{1}\right), S_{1}\left(x^{2} v\right)\right\rangle}{3\left\langle v_{1}, v_{1}\right\rangle} \cdot \operatorname{Re}(X \bar{Y}) .
\end{aligned}
$$


Then, substituting (3.31), (3.32), (3.33) and (3.34) into (3.30), we have

$$
\begin{aligned}
0=\left\langle T_{2} f, f\right\rangle & =\left(\frac{32 \cdot(5 !) \cdot\left\langle D_{2} S_{1}\left(T_{0} v_{1}\right), S_{1}\left(T_{0} v_{1}\right)\right\rangle}{\left\langle v_{1}, v_{1}\right\rangle^{2}}-40\right) \cdot|X|^{2} \\
& +\frac{8 \cdot(6 !)}{\|V\|_{L^{1}}^{2}}\left(6\left\langle D_{2} S_{1}\left(x^{2} v\right), S_{1}\left(x^{2} v\right)\right\rangle-1\right) \cdot|Y|^{2}-\frac{32 \cdot(6 !)\left\langle D_{2} S_{1}\left(T_{0} v_{1}\right), S_{1}\left(x^{2} v\right)\right\rangle}{\left\langle v_{1}, v_{1}\right\rangle \cdot\|V\|_{L^{1}}} \cdot \operatorname{Re}(X \bar{Y}) \\
& :=a|X|^{2}+b|Y|^{2}+c \operatorname{Re}(X \bar{Y}) .
\end{aligned}
$$

Let $X=X_{1}+i X_{2}$ and $Y=Y_{1}+i Y_{2}$ with $X_{1}, X_{2}, Y_{1}, Y_{2}$ are real. Then we have

$$
0=\left\langle T_{2} f, f\right\rangle=a\left(X_{1}^{2}+X_{2}^{2}\right)+b\left(Y_{1}^{2}+Y_{2}^{2}\right)+c\left(X_{1} Y_{1}+X_{2} Y_{2}\right)=\sum_{k=1}^{2}\left(a X_{k}^{2}+b Y_{k}^{2}+c X_{k} Y_{k}\right) .
$$

Hence, without loss of generality, we only need to consider the case that $X$ and $Y$ are real, that is,

$$
0=\left\langle T_{2} f, f\right\rangle=a X^{2}+b Y^{2}+c X Y .
$$

Let $g_{1}=S_{1}\left(x^{2} v\right), h_{1}=D_{2} g_{1} \in S_{1} L^{2}(\mathbf{R}), g_{2}=S_{1}\left(T_{0} v_{1}\right), h_{2}=D_{2} g_{2}$, since $D_{2}=\left(T_{1}+S_{2}\right)^{-1}$, using $S_{1}(v(x))=S_{1}(x v(x))=0$, we have

$$
g_{1}=\left(T_{1}+S_{2}\right) h_{1}=12\left\langle g_{1}, h_{1}\right\rangle g_{1}+192 \frac{\left\langle g_{2}, h_{1}\right\rangle}{\left\langle v_{1}, v_{1}\right\rangle^{2}} g_{2}+S_{2} h_{1}
$$

and

$$
g_{2}=12\left\langle g_{1}, h_{2}\right\rangle g_{1}+192 \frac{\left\langle g_{2}, h_{2}\right\rangle}{\left\langle v_{1}, v_{1}\right\rangle^{2}} g_{2}+S_{2} h_{2}
$$

Then we have

$$
\left\langle D_{2} S_{1}\left(x^{2} v\right), S_{1}\left(x^{2} v\right)\right\rangle=\left\langle g_{1}, h_{1}\right\rangle=12\left\langle g_{1}, h_{1}\right\rangle^{2}+192 \frac{\left\langle g_{2}, h_{1}\right\rangle^{2}}{\left\langle v_{1}, v_{1}\right\rangle^{2}}+\left\langle S_{2} h_{1}, h_{1}\right\rangle
$$

since $\left\langle S_{2} h_{1}, h_{1}\right\rangle \geq 0$ and $\frac{\left\langle g_{2}, h_{1}\right\rangle^{2}}{\left\langle v_{1}, v_{1}\right\rangle^{2}} \geq 0$, we have

$$
\left\langle g_{1}, h_{1}\right\rangle \geq 12\left\langle g_{1}, h_{1}\right\rangle^{2}
$$

thus, we obtain that $\left\langle g_{1}, h_{1}\right\rangle \leq \frac{1}{12}$. Furthermore, if $\left\langle g_{2}, h_{1}\right\rangle \neq 0$, then $\left\langle g_{1}, h_{1}\right\rangle<\frac{1}{12}$.

Similarly, we have

$$
\left\langle D_{2} S_{1}\left(T_{0} v_{1}\right), S_{1}\left(T_{0} v_{1}\right)\right\rangle=\left\langle g_{2}, h_{2}\right\rangle=12\left\langle g_{1}, h_{2}\right\rangle^{2}+192 \frac{\left\langle g_{2}, h_{2}\right\rangle^{2}}{\left\langle v_{1}, v_{1}\right\rangle^{2}}+\left\langle S_{2} h_{2}, h_{2}\right\rangle,
$$

thus we also have

$$
\left\langle g_{2}, h_{2}\right\rangle \leq \frac{\left\langle v_{1}, v_{1}\right\rangle^{2}}{192}
$$

and if $\left\langle g_{1}, h_{2}\right\rangle \neq 0$, we have $\left\langle g_{2}, h_{2}\right\rangle<\frac{\left\langle v_{1}, v_{1}\right\rangle^{2}}{192}$.

Hence, we obtain that

$$
\begin{aligned}
& a=\left(\frac{32 \cdot(5 !) \cdot\left\langle D_{2} S_{1}\left(T_{0} v_{1}\right), S_{1}\left(T_{0} v_{1}\right)\right\rangle}{\left\langle v_{1}, v_{1}\right\rangle^{2}}-40\right) \leq \frac{32 \cdot(5 !)}{192}-40=-20<0, \\
& b=\left(6\left\langle D_{2} S_{1}\left(x^{2} v\right), S_{1}\left(x^{2} v\right)\right\rangle-1\right) \leq \frac{6}{12}-1=-\frac{1}{2}<0 .
\end{aligned}
$$

Furthermore, by Cauchy-Schwarz inequality, we obtain that

$$
\left\langle g_{1}, h_{2}\right\rangle^{2}=\left\langle g_{1}, D_{2} g_{2}\right\rangle^{2} \leq\left\langle g_{1}, D_{2} g_{1}\right\rangle\left\langle g_{2}, D_{2} g_{2}\right\rangle=\left\langle g_{1}, h_{1}\right\rangle\left\langle g_{2}, h_{2}\right\rangle .
$$


Thus

$$
\begin{aligned}
c^{2}-4 a b & =\frac{32^{2} \cdot(6 !)^{2}\left\langle g_{1}, h_{2}\right\rangle^{2}}{\left\langle v_{1}, v_{1}\right\rangle^{2} \cdot\|V\|_{L^{1}}^{2}}-\left(\frac{32 \cdot(5 !) \cdot\left\langle g_{2}, h_{2}\right\rangle}{\left\langle v_{1}, v_{1}\right\rangle^{2}}-40\right) \cdot \frac{32 \cdot(6 !)}{\|V\|_{L^{1}}^{2}}\left(6\left\langle g_{1}, h_{1}\right\rangle-1\right) \\
& \leq \frac{32 \cdot(6 !)}{\|V\|_{L^{1}}^{2}}\left(\frac{32 \cdot(5 !) \cdot\left\langle g_{2}, h_{2}\right\rangle}{\left\langle v_{1}, v_{1}\right\rangle^{2}}+240\left\langle g_{1}, h_{1}\right\rangle-40\right) \\
& \leq \frac{32 \cdot(6 !)}{\|V\|_{L^{1}}^{2}}\left(\frac{32 \cdot(5 !)}{192}+\frac{240}{12}-40\right)=0 .
\end{aligned}
$$

Since "=" holds if and only if $\left\langle g_{1}, h_{2}\right\rangle=\left\langle g_{2}, h_{1}\right\rangle=0$, and in this case, we have

$$
0=\left\langle T_{2} f, f\right\rangle \leq a X^{2}+b Y^{2} \leq 0,
$$

thus it is necessary that

$$
X=\left\langle f, x^{3} v\right\rangle=0, \quad Y=\left\langle T_{0} f, v\right\rangle=0 .
$$

Then $f \in S_{3} L^{2}(\mathbf{R})$. On the other hand, it is obvious that if $f \in S_{3} L^{2}(\mathbf{R})$, we can obtain that $T_{2} f=0$, which means that $f \in \operatorname{ker} T_{2}$.

Lemma 3.5. $S_{3} L^{2}(\mathbf{R})=\{0\}$.

Proof. The proof can be divided into the following two steps.

Step I: We first show that $f(x) \in S_{3} L^{2}(\mathbf{R})$ if and only if $f(x)=U v \phi$ with some $\phi \in L^{2}(\mathbf{R})$ such that $H \phi=0$ in the distributional sense. Furthermore, $\phi=-G_{0} v f$.

Since $S_{3} \leq S_{2}$, by Theorem 4.1(ii) below, we have known that if $f \in S_{2} L^{2}(\mathbf{R})$, then $f=U v \phi$ with $\phi \in W_{\frac{1}{2}}(\mathbf{R})$ such that $H \phi=0$ in the distributional sense and

$$
\phi=-G_{0} v f+\frac{\left\langle v, T_{0} f\right\rangle}{\|V\|_{L^{1}}} .
$$

Thus, we just need to prove that $\frac{\left\langle T_{0} f, v\right\rangle}{\|V\|_{L^{1}(\mathbf{R})}}=0$ and $\phi \in L^{2}(\mathbf{R})$. Indeed, if $f \in S_{3} L^{2}(\mathbf{R})$, then

$$
\int_{\mathbf{R}} v(y) f(y) d y=\int_{\mathbf{R}} y v(y) f(y) d y=\int_{\mathbf{R}} y^{2} v(y) f(y) d y=\int_{\mathbf{R}} y^{3} v(y) f(y) d y=Q T_{0} f=P T_{0} f=0 .
$$

Thus, we have $\frac{\left\langle v, T_{0} f\right\rangle}{\|V\|_{L^{1}}}=0$.

Next we show that $\phi \in L^{2}(\mathbf{R})$. Since $\phi=-G_{0} v f$, and using

$$
S_{3}(v)=S_{3}(x v)=S_{3}\left(x^{2} v\right)=S_{3}\left(x^{3} v\right)=0,
$$

we have

$$
\begin{aligned}
\phi(x) & =-\frac{1}{12} \int_{\mathbf{R}}\left(|x-y|^{3}-|x|^{3}+3|x| x \cdot y-3|x| y^{2}+\frac{x}{|x|} y^{3}\right) v(y) f(y) d y \\
& :=-\frac{1}{12} \int_{\mathbf{R}} K_{3}(x, y) v(y) f(y) d y,
\end{aligned}
$$

where

$$
K_{3}(x, y)=|x-y|^{3}-|x|^{3}+3|x| x \cdot y-3|x| y^{2}+\frac{x}{|x|} y^{3}
$$


Indeed, we can divided $K_{3}(x, y)$ into five parts as follows

$$
\begin{aligned}
K_{3}(x, y)= & {\left[y^{2}(|x-y|-|x|)+\frac{x}{|x|} y^{3}\right]+\left[\frac{x}{|x|} y^{3}-\frac{2 x y^{3}}{|x-y|+|x|}\right] } \\
& +\frac{5}{2}\left[\frac{|x| y^{2}(|x|-|x-y|)}{|x-y|+|x|}-\frac{1}{2} \frac{x}{|x|} y^{3}\right]+\left[\frac{x^{2} y^{2}(|x-y|-|x|)}{(|x-y|+|x|)^{2}}+\frac{1}{4} \frac{x}{|x|} y^{3}\right] \\
& +\frac{|x| y^{4}}{2(|x-y|+|x|)^{2}} \\
:= & K_{31}(x, y)+K_{32}(x, y)+K_{33}(x, y)+K_{34}(x, y)+K_{35}(x, y) .
\end{aligned}
$$

For the first part $K_{31}(x, y)$, we have

$$
\begin{aligned}
\left|K_{31}(x, y)\right| & =\left|\frac{|x| y^{2}\left(y^{2}-2 x \cdot y\right)}{(|x-y|+|x|)|x|}+\frac{x y^{3}(|x-y|+|x|)}{(|x-y|+|x|)|x|}\right| \\
& \lesssim \frac{y^{4}}{|x-y|+|x|}+\left|\frac{x y^{3}(|x-y|-|x|)}{(|x-y|+|x|)|x|}\right| \\
& \lesssim \frac{y^{4}}{|x-y|+|x|} .
\end{aligned}
$$

Moreover,

$$
\left|K_{31}(x, y)\right| \lesssim \frac{y^{4}}{|x-y|+|x|} \lesssim \begin{cases}|y|^{3}, & 0 \leq|x| \leq 1 \\ \frac{y^{4}}{|x|}, & |x|>1\end{cases}
$$

Thus

$$
\left|\int_{\mathbf{R}} K_{31}(x, y) v(y) f(y) d y\right| \lesssim \int_{\mathbf{R}} \frac{y^{4}}{|x-y|+|x|}|v(y) f(y)| d y \in L^{2}(\mathbf{R}) .
$$

For the second part $K_{32}(x, y)$, we have

$$
\begin{aligned}
\left|K_{32}(x, y)\right| & =\left|\frac{x|x| y^{3}+x y^{3}|x-y|-2|x| x y^{3}}{|x|(|x-y|+\mid x) \mid}\right| \\
& =\left|\frac{x y^{3}(|x-y|-|x|)}{|x|(|x-y|+\mid x) \mid}\right| \lesssim \frac{y^{4}}{|x-y|+|x|}
\end{aligned}
$$

Thus

$$
\left|\int_{\mathbf{R}} K_{32}(x, y) v(y) f(y) d y\right| \lesssim \int_{\mathbf{R}} \frac{y^{4}}{|x-y|+|x|}|v(y) f(y)| d y \in L^{2}(\mathbf{R}) .
$$

For the third part $K_{33}(x, y)$, we have

$$
\begin{aligned}
\left|K_{33}(x, y)\right| & =\frac{5}{2}\left|\frac{x^{2} y^{2}\left(2 x \cdot y-y^{2}\right)}{|x|(|x-y|+|x|)^{2}}-\frac{1}{2} \frac{x y^{3}\left(2|x-y| \cdot|x|+2 x^{2}-2 x \cdot y+y^{2}\right)}{|x|(|x-y|+|x|)^{2}}\right| \\
& \lesssim \frac{|x||y|^{5}}{|x|(|x-y|+|x|)^{2}}+\left|\frac{x y^{3}(|x-y|-|x|)}{(|x-y|+|x|)^{2}}\right| \\
& \lesssim \frac{|x| y^{4}}{(|x-y|+|x|)^{2}} .
\end{aligned}
$$

Noting that

$$
\left|K_{33}(x, y)\right| \lesssim \frac{|x| y^{4}}{(|x-y|+|x|)^{2}} \lesssim \begin{cases}|y|^{3}, & 0 \leq|x| \leq 1 \\ \frac{y^{4}}{|x|}, & |x|>1 .\end{cases}
$$

Thus

$$
\left|\int_{\mathbf{R}} K_{33}(x, y) v(y) f(y) d y\right| \lesssim \int_{\mathbf{R}} \frac{|x| y^{4}}{(|x-y|+|x|)^{2}}|v(y) f(y)| d y \in L^{2}(\mathbf{R}) .
$$


Then, for the fourth part $K_{34}(x, y)$, we have

$$
\begin{aligned}
\left|K_{34}(x, y)\right|= & \left|\frac{|x| x^{2} y^{2}\left(y^{2}-2 x \cdot y\right)}{|x|(|x-y|+|x|)^{3}}+\frac{x y^{3}(|x-y|+|x|)^{3}}{4|x|(|x-y|+|x|)^{3}}\right| \\
\lesssim & \frac{x^{2} y^{4}}{(|x-y|+|x|)^{3}}+\frac{|x| \cdot|y|^{5}|x-y|}{(|x-y|+|x|)^{3} \cdot|x|} \\
& +\frac{|x| \cdot|y|^{5}}{(|x-y|+|x|)^{3}}+\left|\frac{x^{3} y^{3}(|x-y|-|x|)}{(|x-y|+|x|)^{3}|x|}\right| \\
\lesssim & \frac{x^{2} y^{4}}{(|x-y|+|x|)^{3}}+\frac{|y|^{5}}{(|x-y|+|x|)^{2}}+\frac{|x| \cdot|y|^{5}}{(|x-y|+|x|)^{3}},
\end{aligned}
$$

and it is obvious that

$$
\frac{x^{2} y^{4}}{(|x-y|+|x|)^{3}}+\frac{|y|^{5}}{(|x-y|+|x|)^{2}}+\frac{|x| \cdot|y|^{5}}{(|x-y|+|x|)^{3}} \lesssim \begin{cases}|y|^{3}, & 0 \leq|x| \leq 1 \\ \frac{y^{4}}{|x|}, & |x|>1\end{cases}
$$

Thus again

$$
\begin{aligned}
& \left|\int_{\mathbf{R}} K_{34}(x, y) v(y) f(y) d y\right| \\
& \lesssim \int_{\mathbf{R}}\left[\frac{x^{2} y^{4}}{(|x-y|+|x|)^{3}}+\frac{|y|^{5}}{(|x-y|+|x|)^{2}}+\frac{|x| \cdot|y|^{5}}{(|x-y|+|x|)^{3}}\right]|v(y) f(y)| d y \in L^{2}(\mathbf{R}) .
\end{aligned}
$$

Finally, for the fifth part $K_{35}(x, y)$, using (3.35), it is easy to know that

$$
\left|\int_{\mathbf{R}} K_{35}(x, y) v(y) f(y) d y\right|=\int_{\mathbf{R}} \frac{|x| y^{4}}{2(|x-y|+|x|)^{2}}|v(y) f(y)| d y \in L^{2}(\mathbf{R}) .
$$

Thus, we obtain that $\phi=-G_{0} v f \in L^{2}(\mathbf{R})$, and $\int_{\mathbf{R}} K_{3}(x, y) v(y) f(y) d y \in L^{2}(\mathbf{R})$.

On the other hand, if $\phi \in L^{2}(\mathbf{R})$ such that $H \phi=0$. Denote $f=U v \phi$, using the similar method of (4.2) and (4.6), we have

$$
\begin{gathered}
\int_{\mathbf{R}} v(y) f(y) d y=0, \int_{\mathbf{R}} y v(y) f(y) d y=0, \int_{\mathbf{R}} y^{2} v(y) f(y) d y=0, \\
\int_{\mathbf{R}} y^{3} v(y) f(y) d y=0, S_{0} T_{0} S_{0} f=0,
\end{gathered}
$$

then we can obtain that $f \in S_{1} L^{2}(\mathbf{R})$ and $G_{0} v f \in L^{2}(\mathbf{R})$. Moreover, by Theorem 4.1, we know that $\phi=-G_{0} v f+c_{1} x+c_{2}$ since $L^{2}(\mathbf{R}) \subset W_{\frac{3}{2}}(\mathbf{R})$. Thus we need that $c_{1} x+c_{2}=\phi+G_{0} v f \in W_{\frac{1}{2}}(\mathbf{R})$ this necessitates that $c_{1}=c_{2}=0$, which implies that $T_{0} f=0$ and $\phi=-G_{0} v f$. Hence, we have $f \in S_{3} L^{2}(\mathbf{R})$.

2Step II: Now we turn to prove that zero is not an eigenvalue of $H$ for $V(x)$ satisfies the condition in Theorem 1.3.

Let $\phi(x) \in L^{2}(\mathbf{R})$ such that $H \phi(x)=0$. Denote $f=U v \phi$, by the Step I, then it follows that $f \in S_{3} L^{2}(\mathbf{R})$ and

$$
\begin{aligned}
L^{2}(\mathbf{R}) \ni \phi(x)=-G_{0} v f & =-\frac{1}{12} \int_{\mathbf{R}}|y-x|^{3} v(y) f(y) d y \\
& =-\frac{1}{12}\left(\int_{x}^{\infty}(y-x)^{3} v(y) f(y) d y+\int_{-\infty}^{x}(x-y)^{3} v(y) f(y) d y\right) .
\end{aligned}
$$

\footnotetext{
${ }^{2}$ The proof was suggested to us by M. Goldberg Gold21]
} 
Then using the following orthogonality relationships from $S_{3} L^{2}(\mathbf{R})$ :

$$
\int_{\mathbf{R}} v(y) f(y) d y=0, \int_{\mathbf{R}} y v(y) f(y) d y=0, \int_{\mathbf{R}} y^{2} v(y) f(y) d y=0, \int_{\mathbf{R}} y^{3} v(y) f(y) d y=0,
$$

and $v(y) f(y)=V(y) \phi(y)$, we have

$$
\phi(x)=-\frac{1}{6} \int_{x}^{\infty}(y-x)^{3} v(y) f(y) d y=-\frac{1}{6} \int_{x}^{\infty}(y-x)^{3} V(y) \phi(y) d y .
$$

Based on the decay condition of Theorem [1.3, that is, $|V(x)| \lesssim\langle x\rangle^{-\beta}$ for $\beta>25$, and $\langle x\rangle=$ $\left(1+|x|^{2}\right)^{1 / 2}$, then $\lim _{x \rightarrow+\infty}|\phi(x)|=0$ and for any $x_{0}>x$,

$$
|\phi(x)| \leq \frac{1}{6} \int_{x}^{x_{0}}(y-x)^{3}|V(y)| \cdot|\phi(y)| d y+\left(\sup _{y \in\left(x_{0},+\infty\right)}|\phi(y)|\right) \int_{x_{0}}^{+\infty} \frac{1}{6}(y-x)^{3}|V(y)| d y .
$$

Hence, we have

$$
\langle x\rangle^{-3}|\phi(x)| \lesssim \int_{x}^{x_{0}}\langle y\rangle^{6}|V(y)| \cdot\langle y\rangle^{-3}|\phi(y)| d y+\left(\sup _{y \in\left(x_{0},+\infty\right)}|\phi(y)|\right) \int_{x_{0}}^{+\infty}\langle y\rangle^{3}|V(y)| d y .
$$

Let

$$
u(x)=\langle x\rangle^{-3}|\phi(x)|, \quad B(y)=\langle y\rangle^{6}|V(y)|
$$

and

$$
A\left(x_{0}\right)=\left(\sup _{y \in\left(x_{0},+\infty\right)}|\phi(y)|\right) \int_{x_{0}}^{+\infty}\langle y\rangle^{3}|V(y)| d y
$$

Then we have

$$
u(x) \lesssim \int_{x}^{x_{0}} B(y) u(y) d y+A\left(x_{0}\right) .
$$

Hence by Gronwall's inequality ( see e.g. [Tao06, Theorem 1.10] ), we conclude that

$$
u(x) \lesssim A\left(x_{0}\right) e^{C \int_{x}^{x_{0}} B(y) d y} .
$$

Note that $\langle y\rangle^{3}|V(y)| \in L^{1}(\mathbf{R})$, hence combining with $\lim _{y \rightarrow+\infty} \phi(y)=0$, we can obtain that $\lim _{x_{0} \rightarrow+\infty} A\left(x_{0}\right)=$ 0. Moreover, $\int_{x}^{x_{0}} B(y) d y$ can be bounded by a fixed constant. Hence for any $x \in \mathbf{R}$,

$$
u(x) \lesssim \lim _{x_{0} \rightarrow+\infty} A\left(x_{0}\right) e^{C \int_{x}^{x_{0}} B(y) d y}=0,
$$

which implies $\phi(x) \equiv 0$. That is, zero can not be an eigenvalue of $H$.

Combined Step I with Step II, we can conclude that $S_{3} L^{2}(\mathbf{R})=\{0\}$.

Remark 3.6. Since the proof of Step II depends on the orthogonality relationships of $S_{3} L^{2}(\mathbf{R})$, so we need the decay rate $\beta$ of $V$ satisfies (1.5). However, we remark that the required decay rate $\beta$ in the proof above may not be optimal to ensure the absence of zero eigenvalue of $H$. In fact, in view of Remark 1.2, it is reasonable to guess that $\beta>4$ might be the best one.

In the following, we come to show the proof of Theorem 1.8 case by case. For the convenience, we will use the notation $A_{k j}^{i}(i$ means the kind of zero resonance, $k$ respects to the power of $\mu$ ) as some bounded operators in $L^{2}(\mathbf{R})$, and these operators may vary from line to line.

Proof of Theorem 1.8; We prove the assertion for + sign. By Lemma 3.3, we know that $\widetilde{M}^{+}(\mu)$ is invertible on $L^{2}(\mathbf{R})$ if and only if

$$
M_{1}^{+}(\mu):=Q-Q\left(\widetilde{M^{+}}(\mu)+Q\right)^{-1} Q
$$


is invertible on $Q L^{2}(\mathbf{R})$. Moreover, using (3.14) and by the definition of $B_{1}^{+}$, we get

$$
\begin{aligned}
M_{1}^{+}(\mu) & =\sum_{k=1}^{12} \mu^{k+1} Q B_{k}^{+} Q+Q \Gamma_{14}(\mu) Q \\
& =\frac{a_{-1}^{+}}{\widetilde{a}^{+}} \mu^{2}\left(Q v G_{-1} v Q+\frac{\widetilde{a}^{+}}{a_{1}^{+}} \sum_{k=2}^{12} \mu^{k-1} Q B_{k}^{+} Q+Q \Gamma_{12}(\mu) Q\right) \\
& :=\frac{a_{-1}^{+}}{\widetilde{a}^{+}} \mu^{2} \widetilde{M}_{1}^{+}(\mu) .
\end{aligned}
$$

Thus, in order to compute the expansions of $\left(M_{1}^{+}(\mu)\right)^{-1}$, we need to obtain the expansions of $\left(\widetilde{M_{1}^{+}}(\mu)\right)^{-1}$ as $\mu \rightarrow 0$ on $Q L^{2}(\mathbf{R})$.

By Lemma 3.4(1), we know that $Q v G_{-1} v Q$ is not invertible on $Q L^{2}(\mathbf{R})$. Since $S_{0}$ be the Riesz projection onto the kernel of $Q v G_{-1} v Q$, then $Q v G_{-1} v Q+S_{0}$ is invertible on $Q L^{2}(\mathbf{R})$. By using (3.37), we have

$$
\widetilde{M_{1}^{+}}(\mu)+S_{0}=\left(Q v G_{-1} v Q+S_{0}\right)+\frac{\widetilde{a}^{+}}{a_{1}^{+}} \sum_{k=2}^{12} \mu^{k-1} Q B_{k}^{+} Q+Q \Gamma_{12}(\mu) Q .
$$

Then, using the Neumann series, we obtain that

$$
\left(\widetilde{M_{1}^{+}}(\mu)+S_{0}\right)^{-1}=D_{0}-\sum_{k=1}^{11} \mu^{k} B_{k}^{0}+D_{0} \Gamma_{12}(\mu) D_{0},
$$

where $B_{k}^{0}(1 \leq k \leq 11)$ are bounded operators in $Q L^{2}(\mathbf{R})$ and we have

$$
\begin{aligned}
B_{1}^{0}= & \frac{\widetilde{a}^{+}}{a_{-1}^{+}} D_{0} B_{2}^{+} D_{0}, \quad B_{2}^{0}=\frac{\widetilde{a}^{+}}{a_{-1}^{+}} D_{0} B_{3}^{+} D_{0}-\frac{\left(\widetilde{a}^{+}\right)^{2}}{\left(a_{-1}^{+}\right)^{2}} D_{0}\left(B_{2}^{+} D_{0}\right)^{2} \\
B_{3}^{0}= & \frac{\widetilde{a}^{+}}{a_{-1}^{+}} D_{0} B_{4}^{+} D_{0}+\frac{\left(\widetilde{a}^{+}\right)^{3}}{\left(a_{-1}^{+}\right)^{3}} D_{0}\left(B_{2}^{+} D_{0}\right)^{3}-\frac{\left(\widetilde{a}^{+}\right)^{2}}{\left(a_{-1}^{+}\right)^{2}}\left(D_{0} B_{2}^{+} D_{0} B_{3}^{+} D_{0}+D_{0} B_{3}^{+} D_{0} B_{2}^{+} D_{0}\right) \\
B_{4}^{0}= & \frac{\widetilde{a}^{+}}{a_{-1}^{+}} D_{0} B_{5}^{+} D_{0}-\frac{\left(\widetilde{a}^{+}\right)^{4}}{\left(a_{-1}^{+}\right)^{4}} D_{0}\left(B_{2}^{+} D_{0}\right)^{4}+\frac{\left(\widetilde{a}^{+}\right)^{3}}{\left(a_{-1}^{+}\right)^{3}}\left(D_{0}\left(B_{2}^{+} D_{0}\right)^{2} B_{3}^{+} D_{0}+D_{0} B_{2}^{+} D_{0} B_{5}^{+} D_{0} B_{2}^{+} D_{0}\right. \\
& \left.+D_{0} B_{3}^{+} D_{0}\left(B_{2}^{+} D_{2}\right)^{2}\right)-\frac{\left(\widetilde{a}^{+}\right)^{2}}{\left(a_{-1}^{+}\right)^{2}}\left(D_{0} B_{2}^{+} D_{0} B_{4}^{+} D_{0}+D_{0} B_{4}^{+} D_{0} B_{2}^{+} D_{0}+D_{0}\left(B_{3}^{+} D_{0}\right)^{2}\right) \\
B_{5}^{0}= & \frac{\widetilde{a}^{+}}{a_{-1}^{+}} D_{0} B_{6}^{+} D_{0}+\frac{\left(\widetilde{a}^{+}\right)^{5}}{\left(a_{-1}^{+}\right)^{5}} D_{0}\left(B_{2}^{+} D_{0}\right)^{5} \\
& -\frac{\left(\widetilde{a}^{+}\right)^{4}}{\left(a_{-1}^{+}\right)^{4}}\left(D_{0}\left(B_{2}^{+} D_{0}\right)^{3} B_{3}^{+} D_{0}+D_{0}\left(B_{2}^{+} D_{0}\right)^{2} B_{3}^{+} D_{0} B_{2}^{+} D_{0}+D_{0} B_{2}^{+} D_{0} B_{3}^{+} D_{0}\left(B_{2}^{+} D_{0}\right)^{2}\right. \\
+ & \left.D_{0} B_{3}^{+} D_{0}\left(B_{2}^{+} D_{0}\right)^{3}\right)+\frac{\left(\widetilde{a}^{+}\right)^{3}}{\left(a_{-1}^{+}\right)^{3}}\left(D_{0} B_{2}^{+} D_{0}\left(B_{3}^{+} D_{0}\right)^{2}+D_{0} B_{3}^{+} D_{0} B_{2}^{+} D_{0} B_{3}^{+} D_{0}+D_{0}\left(B_{3}^{+} D_{0}\right)^{2} B_{2}^{+} D_{0}\right. \\
& \left.+D_{0}\left(B_{2}^{+} D_{0}\right)^{2} B_{4}^{+} D_{0}+D_{0} B_{2}^{+} D_{0} B_{4}^{+} D_{0} B_{2}^{+} D_{0}+D_{0} B_{4}^{+} D_{0}\left(B_{2}^{+} D_{0}\right)^{2}\right) \\
& -\frac{\left(\widetilde{a}^{+}\right)^{2}}{\left(a_{-1}^{+}\right)^{2}}\left(D_{0} B_{3}^{+} D_{0} B_{4}^{+} D_{0}+D_{0} B_{4}^{+} D_{0} B_{3}^{+} D_{0}+D_{0} B_{2}^{+} D_{0} B_{5}^{+} D_{0}+D_{0} B_{5}^{+} D_{0} B_{2}^{+} D_{0}\right)
\end{aligned}
$$

Using Lemma 3.3 again, we know that $\widetilde{M_{1}^{+}}(\mu)$ invertible on $Q L^{2}(\mathbf{R})$ if and only if

$$
M_{2}^{+}(\mu):=S_{0}-S_{0}\left(\widetilde{M_{1}^{+}}(\mu)+S_{0}\right)^{-1} S_{0}
$$


invertible on $S_{0} L^{2}(\mathbf{R})$. Notice that $S_{0} D_{0}=D_{0} S_{0}=S_{0}$, and using (3.39), we obtain that

$$
\begin{aligned}
M_{2}^{+}(\mu) & =\mu S_{0} B_{1}^{0} S_{0}+\sum_{k=2}^{11} \mu^{k} B_{k}^{0}+S_{0} \Gamma_{12}(\mu) S_{0} \\
& =\frac{\mu}{a_{-1}^{+}}\left(S_{0} T_{0} S_{0}+a_{-1}^{+} \sum_{k=2}^{11} \mu^{k-1} B_{k}^{0}+S_{0} \Gamma_{11}(\mu) S_{0}\right) \\
& :=\frac{\mu}{a_{-1}^{+}} \widetilde{M_{2}^{+}}(\mu) .
\end{aligned}
$$

(1) Regular Case. In this case, we using (3.5) which need $|V(x)| \lesssim(1+|x|)^{-13-}$, see Lemma 3.1. Then by the same process, we can rewrite $M_{2}^{+}(\mu)$ as follows:

$$
\begin{aligned}
M_{2}^{+}(\mu) & =\mu S_{0} B_{1}^{0} S_{0}+\mu^{2} B_{2}^{0}+\mu^{3} B_{3}^{0}+S_{0} \Gamma_{4}(\mu) S_{0} \\
& =\frac{\mu}{a_{-1}^{+}}\left(S_{0} T_{0} S_{0}+a_{-1}^{+} \mu B_{2}^{0}+a_{-1}^{+} \mu^{2} B_{3}^{0}+S_{0} \Gamma_{3}(\mu) S_{0}\right) \\
& :=\frac{\mu}{a_{-1}^{+}} \widetilde{M_{2}^{+}}(\mu) .
\end{aligned}
$$

If zero is a regular point of the spectrum of $H$, then $S_{0} T_{0} S_{0}$ is invertible on $S_{0} L^{2}(\mathbf{R})$. Denote $\widetilde{D_{0}}=\left(S_{0} T_{0} S_{0}\right)^{-1}$, by (3.41), using Neumann series and $S_{0} \widetilde{D}_{0}=\widetilde{D}_{0} S_{0}=\widetilde{D}_{0}$, we obtain that

$$
\left(\widetilde{M_{2}^{+}}(\mu)\right)^{-1}=\widetilde{D_{0}}-a_{-1}^{+} \mu \widetilde{D}_{0} B_{2}^{0} \widetilde{D}_{0}+\mu^{2}\left(\left(a_{-1}^{+}\right)^{2} \widetilde{D}_{0}\left(B_{2}^{0} \widetilde{D}_{0}\right)^{2}-a_{-1}^{+} \widetilde{D}_{0} B_{3}^{0} \widetilde{D}_{0}\right)+\Gamma_{3}(\mu)
$$

Then, using (3.41), we have

$$
\left(M_{2}^{+}(\mu)\right)^{-1}=\frac{a_{-1}^{+}}{\mu} \widetilde{D_{0}}-\left(a_{-1}^{+}\right)^{2} \widetilde{D}_{0} B_{2}^{0} \widetilde{D}_{0}+\mu\left(\left(a_{-1}^{+}\right)^{3} \widetilde{D}_{0}\left(B_{2}^{0} \widetilde{D}_{0}\right)^{2}-\left(a_{-1}^{+}\right)^{2} \widetilde{D}_{0} B_{3}^{0} \widetilde{D}_{0}\right)+\Gamma_{2}(\mu) .
$$

By Lemma 3.3 with $A=\widetilde{M_{1}^{+}}(\mu), S=S_{0}$ and $B=M_{2}^{+}(\mu)$, using the orthogonality properties ( see (3.16), (3.17)), we obtain that

$$
\begin{aligned}
\left(\widetilde{M_{1}^{+}}(\mu)\right)^{-1} & =\left(\widetilde{M_{1}^{+}}(\mu)+S_{0}\right)^{-1}+\left(\widetilde{M_{1}^{+}}(\mu)+S_{0}\right)^{-1} S_{0}\left(M_{2}^{+}(\mu)\right)^{-1} S_{0}\left(\widetilde{M_{1}^{+}}(\mu)+S_{0}\right)^{-1} \\
& =\frac{S_{0} A_{-1,1}^{0} S_{0}}{\mu}+Q A_{01}^{0} Q+\mu Q A_{11}^{0} Q+\Gamma_{2}(\mu) .
\end{aligned}
$$

Then, using (3.37), we have

$$
\left(M_{1}^{+}(\mu)\right)^{-1}=\frac{S_{0} A_{-3,1}^{0} S_{0}}{\mu^{3}}+\frac{Q A_{-2,1}^{0} Q}{\mu^{2}}+\frac{Q A_{-1,1}^{0} Q}{\mu}+\Gamma_{0}(\mu) .
$$

Then, we use Lemma 3.3 with $A=\widetilde{M^{+}}(\mu), S=Q$ and $B=M_{1}^{+}(\mu)$, and using the orthogonality properties ( see (3.16), (3.17)), we have

$$
\begin{aligned}
\left(\widetilde{M^{+}}(\mu)\right)^{-1} & =\left(\widetilde{M^{+}}(\mu)+Q\right)^{-1}+\left(\widetilde{M^{+}}(\mu)+Q\right)^{-1} Q\left(M_{1}^{+}(\mu)\right)^{-1} Q\left(\widetilde{M^{+}}(\mu)+Q\right)^{-1} \\
& =\frac{S_{0} A_{-3,1}^{0} S_{0}}{\mu^{3}}+\frac{Q A_{-2,1}^{0} Q}{\mu^{2}}+\frac{Q A_{-1,1}^{0} Q+S_{0} A_{-1,2}^{0}+A_{-1,3}^{0} S_{0}}{\mu}+\Gamma_{0}(\mu) .
\end{aligned}
$$

Finally, since $M^{+}(\mu)=\frac{\widetilde{a}^{+}}{\mu^{3}} \widetilde{M^{+}}(\mu)$, then we can say that

$$
\left(M^{+}(\mu)\right)^{-1}=S_{0} A_{01}^{0} S_{0}+\mu Q A_{11}^{0} Q+\mu^{2}\left(Q A_{21}^{0} Q+S_{0} A_{22}^{0}+A_{23}^{0} S_{0}\right)+\Gamma_{3}^{0}(\mu) .
$$


(2) First Kind of Resonance. In this case, we using (3.6) with $|V(x)| \lesssim(1+|x|)^{-17-}$, see Lemma 3.1. Then by the same process, we can rewrite $M_{2}^{+}(\mu)$ as follows:

$$
\begin{aligned}
M_{2}^{+}(\mu) & =\mu S_{0} B_{1}^{0} S_{0}+\sum_{k=2}^{5} \mu^{k} B_{k}^{0}+S_{0} \Gamma_{6}(\mu) S_{0} \\
& =\frac{\mu}{a_{-1}^{+}}\left(S_{0} T_{0} S_{0}+a_{-1}^{+} \sum_{k=2}^{5} \mu^{k-1} B_{k}^{0}+S_{0} \Gamma_{5}(\mu) S_{0}\right) \\
& :=\frac{\mu}{a_{-1}^{+}} \widetilde{M_{2}^{+}}(\mu) .
\end{aligned}
$$

For the first kind of resonance, we aim to derive the expansions of $\left(\widetilde{M_{2}^{+}}(\mu)\right)^{-1}$. By the definition of the first kind of resonance, we know that the operator $S_{0} T_{0} S_{0}$ is not invertible on $S_{0} L^{2}(\mathbf{R})$. Since $S_{1}$ is the Riesz projection onto the kernel of $S_{0} T_{0} S_{0}$ on $S_{0} L^{2}(\mathbf{R})$, thus $S_{0} T_{0} S_{0}+S_{1}$ is invertible on $S_{0} L^{2}(\mathbf{R})$. By Neumann series, we have

$$
\left(\widetilde{M_{2}^{+}}(\mu)+S_{1}\right)^{-1}=D_{1}-\sum_{k=1}^{4} \mu^{k} B_{k}^{1}+\Gamma_{5}(\mu),
$$

where $B_{k}^{1}(1 \leq k \leq 4)$ are bounded operators in $S_{0} L^{2}(\mathbf{R})$ with the following definition

$$
\begin{aligned}
B_{1}^{1}= & a_{-1}^{+} D_{1} B_{2}^{0} D_{1}, \quad B_{2}^{1}=a_{-1}^{+} D_{1} B_{3}^{0} D_{1}-\left(a_{-1}^{+}\right)^{2} D_{1}\left(B_{2}^{0} D_{1}\right)^{2}, \\
B_{3}^{1}= & a_{-1}^{+} D_{1} B_{4}^{0} D_{1}+\left(a_{-1}^{+}\right)^{3} D_{1}\left(B_{1}^{0} D_{1}\right)^{3}-\left(a_{-1}^{+}\right)^{2}\left(D_{1} B_{2}^{0} D_{1} B_{3}^{0} D_{1}+D_{1} B_{3}^{0} D_{1} B_{2}^{0} D_{1}\right), \\
B_{4}^{1}= & \left(a_{-1}^{+}\right) D_{1} B_{5}^{0} D_{1}-\left(a_{-1}^{+}\right)^{4} D_{1}\left(B_{2}^{0} D_{1}\right)^{4} \\
& +\left(a_{-1}^{+}\right)^{3}\left(D_{1}\left(B_{2}^{0} D_{1}\right)^{2} B_{3}^{0} D_{1}+D_{1} B_{2}^{0} D_{1} B_{3}^{0} D_{1} B_{2}^{0} D_{1}+D_{1} B_{3}^{0} D_{1}\left(B_{2}^{0} D_{1}\right)^{2}\right) \\
& +\left(a_{-1}^{+}\right)^{2}\left(D_{1} B_{2}^{0} D_{1} B_{4}^{0} D_{1}+D_{1} B_{4}^{0} D_{1} B_{2}^{0} D_{1}+D_{1}\left(B_{3}^{0} D_{1}\right)^{2}\right) .
\end{aligned}
$$

According to Lemma 3.3, we know that $\widetilde{M_{2}^{+}}(\mu)$ is invertible on $S_{0} L^{2}(\mathbf{R})$ if and only if

$$
\left.M_{3}^{+}(\mu):=S_{1}-S_{1} \widetilde{M_{2}^{+}}(\mu)+S_{1}\right)^{-1} S_{1}
$$

is invertible on $S_{1} L^{2}(\mathbf{R})$. Moreover, using (3.43) and (3.17), we obtain

$$
M_{3}^{+}(\mu)=\mu S_{1} B_{1}^{1} S_{1}+\mu^{2} S_{1} B_{2}^{1} S_{1}+\mu^{3} S_{1} B_{3}^{1} S_{1}+\mu^{4} S_{1} B_{4}^{1} S_{1}+S_{1} \Gamma_{5}(\mu) S_{1} .
$$

Now, we shall compute the $S_{1} B_{1}^{1} S_{1}$ in $M_{3}^{+}(\mu)$. Noting that $S_{1} D_{1}=D_{1} S_{1}=S_{1}$, we have $S_{1} B_{1}^{1} S_{1}=$ $a_{-1}^{+} S_{1} B_{2}^{0} S_{1}$. Since

$$
\begin{aligned}
B_{2}^{0} & =\frac{\widetilde{a}^{+}}{a_{-1}^{+}} D_{0} B_{3}^{+} D_{0}-\frac{\left(\widetilde{a}^{+}\right)^{2}}{\left(a_{-1}^{+}\right)^{2}} D_{0}\left(B_{2}^{+} D_{0}\right)^{2} \\
& =\frac{a_{1}^{+}}{a_{-1}^{+}} D_{0} v G_{1} v D_{0}-\frac{a_{-1}^{+}}{\widetilde{a}^{+}} D_{0}\left(v G_{-1} v\right)^{2} D_{0}-\frac{1}{\left(a_{-1}^{+}\right)^{2}} D_{0} T_{0} D_{0} T_{0} D_{0}
\end{aligned}
$$

and $S_{1} D_{0}=D_{0} S_{1}=S_{1}$, we have

$$
S_{1} B_{1}^{1} S_{1}=a_{-1}^{+}\left(\frac{a_{1}^{+}}{a_{-1}^{+}} S_{1} v G_{1} v S_{1}-\frac{a_{-1}^{+}}{\widetilde{a}^{+}} S_{1}\left(v G_{-1} v\right)^{2} S_{1}-\frac{1}{\left(a_{-1}^{+}\right)^{2}} S_{1} T_{0} D_{0} T_{0} S_{1}\right) .
$$

Notice that $\left(v G_{-1} v\right)^{2}=v G_{-1} v v G_{-1} v=v G_{-1} v P v G_{-1} v$, we have

$$
S_{1} B_{1}^{1} S_{1}=a_{1}^{+} T_{1},
$$


where

$$
\begin{aligned}
T_{1} & =S_{1} v G_{1} v S_{1}-\frac{\left(a_{-1}^{+}\right)^{2}}{\widetilde{a}^{+} a_{1}^{+}} S_{1} v G_{-1} v P v G_{-1} v S_{1}-\frac{1}{a_{1}^{+} a_{-1}^{+}} S_{1} T_{0} D_{0} T_{0} S_{1} \\
& =S_{1} v G_{1} v S_{1}+\frac{6}{\|V\|_{L^{1}}} S_{1} v G_{-1} v P v G_{-1} v S_{1}-384 S_{1} T_{0} D_{0} T_{0} S_{1} .
\end{aligned}
$$

Recall that (3.46) and (3.48), we can rewrite $M_{3}^{+}(\mu)$ as follows:

$$
\begin{aligned}
M_{3}^{+}(\mu) & =a_{1}^{+} \mu T_{1}+\mu^{2} S_{1} B_{2}^{1} S_{1}+\mu^{3} S_{1} B_{3}^{1} S_{1}+\mu^{4} S_{1} B_{4}^{1} S_{1}+S_{1} \Gamma_{5}(\mu) S_{1} \\
& =a_{1}^{+} \mu\left(T_{1}+\frac{1}{a_{1}^{+}}\left(\mu S_{1} B_{2}^{1} S_{1}+\mu^{2} S_{1} B_{3}^{1} S_{1}+\mu^{3} S_{1} B_{4}^{1} S_{1}\right)+S_{1} \Gamma_{4}(\mu) S_{1}\right) \\
& :=a_{1}^{+} \mu \widetilde{M_{3}^{+}}(\mu) .
\end{aligned}
$$

Since $T_{1}$ is invertible on $S_{1} L^{2}(\mathbf{R})$, we have $S_{1} T_{1}^{-1}=T_{1}^{-1} S_{1}=T_{1}^{-1}$, then using Neumann series, we have the following asymptotic expansions of $\left(\widetilde{M_{3}^{+}}(\mu)\right)^{-1}$ as $\mu \rightarrow 0$ :

$$
\left(\widetilde{M_{3}^{+}}(\mu)\right)^{-1}=S_{1} A_{01}^{1} S_{1}+\mu S_{1} A_{11}^{1} S_{1}+\mu^{2} S_{1} A_{21}^{1} S_{1}+\mu^{3} S_{1} A_{31}^{1} S_{1}+\Gamma_{4}(\mu) .
$$

Then, we have

$$
\left(M_{3}^{+}(\mu)\right)^{-1}=\frac{S_{1} A_{-1,1}^{1} S_{1}}{\mu}+S_{1} A_{01}^{1} S_{1}+\mu S_{1} A_{11}^{1} S_{1}+\mu^{2} S_{1} A_{21}^{1} S_{1}+\Gamma_{3}(\mu) .
$$

Next, by Lemma 3.3 and using (3.17), we have

$$
\begin{aligned}
\left(\widetilde{M_{2}^{+}}(\mu)\right)^{-1} & =\left(\widetilde{M_{2}^{+}}(\mu)+S_{1}\right)^{-1}+\left(\widetilde{M_{2}^{+}}(\mu)+S_{1}\right)^{-1} S_{1}\left(M_{3}^{+}(\mu)\right)^{-1} S_{1}\left(\widetilde{M_{2}^{+}}(\mu)+S_{1}\right)^{-1} \\
& =\frac{S_{1} A_{-1,1}^{1} S_{1}}{\mu}+S_{0} A_{01}^{1} S_{0}+\mu S_{0} A_{11}^{1} S_{0}+\mu^{2} S_{0} A_{21}^{1} S_{0}+\Gamma_{3}(\mu) .
\end{aligned}
$$

Furthermore, we have

$$
\left(M_{2}^{+}(\mu)\right)^{-1}=\frac{S_{1} A_{-2,1}^{1} S_{1}}{\mu^{2}}+\frac{S_{0} A_{-1,1}^{1} S_{0}}{\mu}+S_{0} A_{01}^{1} S_{0}+\mu S_{0} A_{11}^{1} S_{0}+\Gamma_{2}(\mu) .
$$

Then using the orthogonality properties (see (3.16), (3.16)), and following the similar process of Regular Case, we obtain that

$$
\begin{aligned}
\left(M^{+}(\mu)\right)^{-1}= & \frac{S_{1} A_{-1,1}^{1} S_{1}}{\mu}+S_{0} A_{01}^{1} Q+Q A_{02}^{1} S_{0}+\mu\left(Q A_{11}^{1} Q+S_{0} A_{12}^{1}+A_{13}^{1} S_{0}\right) \\
& +\mu^{2}\left(Q A_{21}^{1}+A_{22}^{1} Q\right)+\Gamma_{3}^{1}(\mu) .
\end{aligned}
$$

(3) Second Kind of Resonance. In this case, we using (3.7) with $|V(x)| \lesssim(1+|x|)^{-25-}$, see Lemma 3.1. Then by the same process, we can rewrite $M_{2}^{+}(\mu)$ as follows:

$$
\begin{aligned}
M_{2}^{+}(\mu) & =\mu S_{0} B_{1}^{0} S_{0}+\sum_{k=2}^{8} \mu^{k} B_{k}^{0}+S_{0} \Gamma_{10}(\mu) S_{0} \\
& =\frac{\mu}{a_{-1}^{+}}\left(S_{0} T_{0} S_{0}+a_{-1}^{+} \sum_{k=2}^{5} \mu^{k-1} B_{k}^{0}+S_{0} \Gamma_{9}(\mu) S_{0}\right) \\
& :=\frac{\mu}{a_{-1}^{+}} \widetilde{M_{2}^{+}}(\mu) .
\end{aligned}
$$


Furthermore, we obtain a more detail expansion of $M_{3}^{+}(\mu)$ :

$$
\begin{aligned}
M_{3}^{+}(\mu) & =a_{1}^{+} \mu T_{1}+\sum_{k=2}^{8} \mu^{k} S_{1} B_{k}^{1} S_{1}+S_{1} \Gamma_{9}(\mu) S_{1} \\
& =a_{1}^{+} \mu\left(T_{1}+\frac{1}{a_{1}^{+}}\left(\mu S_{1} B_{2}^{1} S_{1}+\sum_{k=2}^{8} \mu^{k-1} S_{1} B_{k}^{1} S_{1}+S_{1} \Gamma_{8}(\mu) S_{1}\right)\right. \\
& :=a_{1}^{+} \mu \widetilde{M_{3}^{+}}(\mu),
\end{aligned}
$$

where $B_{2}^{1}, B_{3}^{1}, B_{4}^{1}$ are given in (3.44) and $B_{k}^{1}(5 \leq k \leq 8)$ are also bounded operators in $L^{2}(\mathbf{R})$.

For the second kind of resonance, we aim to derive the expansions of $\left(\widetilde{M_{3}^{+}}(\mu)\right)^{-1}$. By the definition of the second kind of resonance, we know that the operator $T_{1}$ is not invertible on $S_{1} L^{2}(\mathbf{R})$. Since $S_{2}$ is the Riesz projection onto the kernel of $T_{1}$ on $S_{1} L^{2}(\mathbf{R})$, thus $T_{1}+S_{2}$ is invertible on $S_{1} L^{2}(\mathbf{R})$. Using Neumann series, we have

$$
\left(\widetilde{M_{3}^{+}}(\mu)+S_{2}\right)^{-1}=D_{2}-\sum_{k=1}^{7} \mu^{k} B_{k}^{2}+\Gamma_{8}(\mu),
$$

where $B_{k}^{2}(1 \leq k \leq 7)$ are bounded operators in $S_{1} L^{2}(\mathbf{R})$, and $B_{1}^{2}, B_{2}^{2}, B_{3}^{2}$ are given as follows:

$$
\begin{aligned}
& B_{1}^{2}=\frac{1}{a_{1}^{+}} D_{2} B_{2}^{1} D_{2}, \quad B_{2}^{2}=\frac{1}{a_{1}^{+}} D_{2} B_{3}^{1} D_{2}-\frac{1}{\left(a_{1}^{+}\right)^{2}} D_{2} B_{2}^{1} D_{2} B_{2}^{1} D_{2}, \\
& B_{3}^{2}=\frac{1}{a_{1}^{+}} D_{2} B_{4}^{1} D_{2}+\frac{1}{\left(a_{1}^{+}\right)^{3}} D_{2} B_{2}^{1} D_{2} B_{2}^{1} D_{2}-\frac{1}{\left(a_{1}^{+}\right)^{2}}\left(D_{2} B_{2}^{1} D_{2} B_{3}^{1} D_{2}+D_{2} B_{3}^{1} D_{2} B_{2}^{1} D_{2}\right) .
\end{aligned}
$$

By Lemma 3.3, we know that $\widetilde{M_{3}^{+}}(\mu)$ is invertible on $S_{1} L^{2}(\mathbf{R})$ if and only if

$$
M_{4}^{+}(\mu):=S_{2}-S_{2}\left(\widetilde{M_{3}^{+}}(\mu)+S_{2}\right)^{-1} S_{2}
$$

is invertible on $S_{2} L^{2}(\mathbf{R})$. Moreover, using (3.51) and noting that $S_{2} D_{2}=D_{2} S_{2}=S_{2}$, we obtain

$$
M_{4}^{+}(\mu)=\sum_{k=1}^{7} \mu^{k} S_{2} B_{k}^{2} S_{2}+\Gamma_{8}(\mu) .
$$

Indeed, using the orthogonal properties of $S_{2}$ (see (3.17)-(3.20) $)$, we obtain that $S_{2} B_{1}^{2} S_{2}=0$ and

$$
\begin{aligned}
S_{2} B_{2}^{2} S_{2}= & \frac{a_{3}^{+}}{a_{1}^{+}}\left(S_{2} v G_{3} v S_{2}-\frac{1}{a_{2}^{+} \widetilde{a}^{+}} S_{2} T_{0}^{2} S_{2}-\frac{\left(a_{1}^{+}\right)^{2}}{a_{-1}^{+} a_{3}^{+}} S_{2} v G_{1} v D_{0} v G_{1} v S_{2}\right. \\
& \left.\quad-\frac{\left(a_{-1}^{+}\right)^{2}}{a_{1}^{+} a_{3}^{+}\left(\widetilde{a}^{+}\right)^{2}} S_{2} T_{0} v G_{-1} v D_{2} v G_{-1} v T_{0} S_{2}\right) \\
= & \frac{a_{3}^{+}}{a_{1}^{+}}\left(S_{2} v G_{3} v S_{2}-\frac{8 \cdot(6 !)}{\|V\|_{L^{1}}} S_{2} T_{0}^{2} S_{2}+\frac{5}{2} S_{2} v G_{1} v D_{0} v G_{1} v S_{2}\right. \\
& \quad+\frac{48 \cdot(6 !)}{\|V\|_{L^{1}}^{2}}\left(S_{2} T_{0} v G_{-1} v-\frac{\|V\|_{L^{1}}}{6} S_{2} v G_{1} v D_{0} T_{0}\right) D_{2}\left(v G_{-1} v T_{0} S_{2}-\frac{\|V\|_{L^{1}}}{6} T_{0} D_{0} v G_{1} v S_{2}\right) \\
:= & \frac{a_{3}^{+}}{a_{1}^{+}} T_{2} .
\end{aligned}
$$


Furthermore, we can rewrite (3.53) as follows:

$$
\begin{aligned}
M_{4}^{+}(\mu) & =\frac{a_{3}^{+}}{a_{1}} \mu^{2} T_{2}+\sum_{k=3}^{7} \mu^{k} S_{2} B_{k}^{2} S_{2}+S_{2} \Gamma_{8}(\mu) S_{2} \\
& =\frac{a_{3}^{+}}{a_{1}^{+}} \mu^{2}\left(T_{2}+\frac{a_{1}^{+}}{a_{2}^{+}} \sum_{k=3}^{7} \mu^{k-2} S_{2} B_{k}^{2} S_{2}+S_{2} \Gamma_{6}(\mu) S_{2}\right) \\
& :=\frac{a_{3}^{+}}{a_{1}^{+}} \mu^{2} \widetilde{M_{4}^{+}}(\mu) .
\end{aligned}
$$

According to Lemma 3.4 and Lemma 3.5, we know that $\operatorname{ker} T_{2}=\{0\}$, which means that $T_{2}$ is invertible on $S_{2} L^{2}(\mathbf{R})$, by Neumann series and using $T_{2}^{-1}=S_{2} T_{2}^{-1}=T_{2}^{-1} S_{2}$, we have the following asymptotic expansions of $\left(\widetilde{M_{4}^{+}}(\mu)\right)^{-1}$ as $\mu \rightarrow 0$ :

$$
\left(\widetilde{M_{4}^{+}}(\mu)\right)^{-1}=S_{2} A_{01}^{2} S_{2}+\sum_{k=1}^{5} \mu^{k} S_{2} A_{k 1}^{2} S_{2}+\Gamma_{6}(\mu) .
$$

Thus we have

$$
\left(M_{4}^{+}(\mu)\right)^{-1}=\frac{S_{2} A_{-2,1}^{2} S_{2}}{\mu^{2}}+\sum_{k=-1}^{3} \mu^{k} S_{2} A_{k 1}^{2} S_{2}+\Gamma_{4}(\mu)
$$

Then by Lemma 3.3 and using the orthogonal relationships (3.17), we have

$$
\begin{aligned}
\left(\widetilde{M_{3}^{+}}(\mu)\right)^{-1} & =\left(\widetilde{M_{3}^{+}}(\mu)+S_{2}\right)^{-1}+\left(\widetilde{M_{3}^{+}}(\mu)+S_{2}\right)^{-1} S_{2}\left(M_{4}^{+}(\mu)\right)^{-1} S_{2}\left(\widetilde{M_{3}^{+}}(\mu)+S_{2}\right)^{-1} \\
& =\frac{S_{2} A_{-2,1}^{2} S_{2}}{\mu^{2}}+\frac{S_{2} A_{-1,1}^{2} S_{1}+S_{1} A_{-1,2}^{2} S_{2}}{\mu}+\sum_{k=0}^{3} \mu^{k} S_{1} A_{k 1}^{2} S_{1}+\Gamma_{4}(\mu) .
\end{aligned}
$$

Next, we can obtain that

$$
\left(M_{3}^{+}(\mu)\right)^{-1}=\frac{S_{2} A_{-3,1}^{2} S_{2}}{\mu^{3}}+\frac{S_{2} A_{-2,1}^{2} S_{1}+S_{1} A_{-2,2}^{2} S_{2}}{\mu^{2}}+\sum_{k=-1}^{2} \mu^{k} S_{1} A_{k 1}^{2} S_{1}+\Gamma_{3}(\mu)
$$

Using the orthogonal relationships (3.17) and Lemma 3.3, we have

$$
\begin{aligned}
\left(\widetilde{M_{2}^{+}}(\mu)\right)^{-1} & =\left(\widetilde{M_{2}^{+}}(\mu)+S_{1}\right)^{-1}+\left(\widetilde{M_{2}^{+}}(\mu)+S_{1}\right)^{-1} S_{1}\left(M_{3}^{+}(\mu)\right)^{-1} S_{1}\left(\widetilde{M_{2}^{+}}(\mu)+S_{1}\right)^{-1} \\
& =\frac{S_{2} A_{-3,1}^{2} S_{2}}{\mu^{3}}+\frac{S_{2} A_{-2,1}^{2} S_{0}+S_{0} A_{-2,2}^{2} S_{2}}{\mu^{2}}+\sum_{k=-1}^{2} \mu^{k} S_{0} A_{k 1}^{2} S_{0}+\Gamma_{3}(\mu) .
\end{aligned}
$$

Furthermore, since $M_{2}^{+}(\mu)=\frac{\mu}{a_{-1}^{+}} \widetilde{M_{2}^{+}}(\mu)$, then

$$
\begin{aligned}
\left(M_{2}^{+}(\mu)\right)^{-1}= & \frac{S_{2} A_{-4,1}^{2} S_{2}}{\mu^{4}}+\frac{S_{2} A_{-3,1}^{2} S_{0}+S_{0} A_{-3,2}^{2} S_{2}}{\mu^{3}}+\frac{S_{0} A_{-2,1}^{2} S_{0}}{\mu^{2}}+\frac{S_{0} A_{-1,1}^{2} S_{0}}{\mu} \\
& +\mu S_{0} A_{01}^{2} S_{0}+\Gamma_{2}(\mu) .
\end{aligned}
$$


Next, using Lemma 3.3 and the orthogonal properties of $S_{2}$ (see (3.16)-(3.20) ), we have

$$
\begin{aligned}
\left(\widetilde{M_{1}^{+}}(\mu)\right)^{-1}= & \left(\widetilde{M_{1}^{+}}(\mu)+S_{0}\right)^{-1}+\left(\widetilde{M_{1}^{+}}(\mu)+S_{0}\right)^{-1} S_{0}\left(M_{2}^{+}(\mu)\right)^{-1} S_{0}\left(\widetilde{M_{1}^{+}}(\mu)+S_{0}\right)^{-1} \\
= & \frac{S_{2} A_{-4,1}^{2} S_{2}}{\mu^{4}}+\frac{S_{2} A_{-3,1}^{2} S_{0}+S_{0} A_{-3,2}^{2} S_{2}}{\mu^{3}}+\frac{S_{0} A_{-2,1}^{2} S_{0}+Q A_{-2,2}^{2} S_{2}+S_{2} A_{-2,3}^{2} Q}{\mu^{2}} \\
& +\frac{Q A_{-1,1}^{2} S_{0}+S_{0} A_{-1,2}^{2} Q}{\mu}+Q A_{01}^{2} Q+\mu Q A_{11}^{2} Q+\Gamma_{2}(\mu) .
\end{aligned}
$$

Since $M_{1}^{+}(\mu)=\frac{a_{-1}^{+}}{\widetilde{a}^{+}} \widetilde{M}_{1}^{+}(\mu)$, then

$$
\begin{aligned}
\left(M_{1}^{+}(\mu)\right)^{-1}= & \frac{S_{2} A_{-6,1}^{2} S_{2}}{\mu^{6}}+\frac{S_{2} A_{-5,1}^{2} S_{0}+S_{0} A_{-5,2}^{2} S_{2}}{\mu^{5}}+\frac{S_{0} A_{-4,1}^{2} S_{0}+Q A_{-4,2}^{2} S_{2}+S_{2} A_{-4,3}^{2} Q}{\mu^{4}} \\
& +\frac{Q A_{-3,1}^{2} S_{0}+S_{0} A_{-3,2}^{2} Q}{\mu^{3}}+\frac{Q A_{-2,1}^{2} Q}{\mu^{2}}+\frac{Q A_{11}^{2} Q}{\mu}+\Gamma_{1}(\mu) .
\end{aligned}
$$

Finally, using the orthogonality relationships (see (3.16)-(3.20) $)$, we have

$$
\begin{aligned}
\left(\widetilde{M^{+}}(\mu)\right)^{-1}= & \left(\widetilde{M^{+}}(\mu)+Q\right)^{-1}+\left(\widetilde{M^{+}}(\mu)+Q\right)^{-1} Q\left(M_{1}^{+}(\mu)\right)^{-1} Q\left(\widetilde{M^{+}}(\mu)+Q\right)^{-1} \\
= & \frac{S_{2} A_{-6,1}^{2} S_{2}}{\mu^{6}}+\frac{S_{2} A_{-5,1}^{2} S_{0}+S_{0} A_{-5,2}^{2} S_{2}}{\mu^{5}}+\frac{S_{0} A_{-4,1}^{2} S_{0}+Q A_{-4,2}^{2} S_{2}+S_{2} A_{-4,3}^{2} Q}{\mu^{4}} \\
& +\frac{Q A_{-3,1}^{2} S_{0}+S_{0} A_{-3,2}^{2} Q+S_{2} A_{-3,3}^{2}+A_{-3,4}^{2} S_{2}}{\mu^{3}}+\frac{Q A_{-2,1}^{2} Q+S_{0} A_{-2,2}^{2}+A_{-2,3}^{2} S_{0}}{\mu^{2}} \\
& +\frac{Q A_{-1,1}^{2}+A_{-1,2}^{2}}{\mu}+\Gamma_{0}(\mu) .
\end{aligned}
$$

Furthermore, we obtain the following asymptotic expansions of $\left(M^{+}(\mu)\right)^{-1}$ for zero is the second resonance of $H$,

$$
\begin{aligned}
\left(M^{+}(\mu)\right)^{-1}= & \frac{S_{2} A_{-3,1}^{2} S_{2}}{\mu^{3}}+\frac{S_{2} A_{-2,1}^{2} S_{0}+S_{0} A_{-2,2}^{2} S_{2}}{\mu^{2}}+\frac{S_{0} A_{-1,1}^{2} S_{0}+Q A_{-1,2}^{2} S_{2}+S_{2} A_{-1,3}^{2} Q}{\mu} \\
& +\left(Q A_{01}^{2} S_{0}+S_{0} A_{02}^{2} Q+S_{2} A_{03}^{2}+A_{04}^{2} S_{2}\right)+\mu\left(Q A_{11}^{2} Q+S_{0} A_{12}^{2}+A_{13} S_{0}\right) \\
& +\mu^{2}\left(Q A_{21}^{2}+A_{21}^{2} Q\right)+\Gamma_{3}^{2}(\mu) .
\end{aligned}
$$

\section{Proof of Theorem 1.7}

In this part, we aim to give the proof of Theorem 1.7. In fact, it suffices to establish the following characterizations of resonance spaces.

Theorem 4.1. Assume that $|V(x)| \lesssim(1+|x|)^{-\beta}$ with some $\beta>17$. Then the following statements hold:

(i) $f(x) \in S_{1} L^{2}(\mathbf{R})$ if and only if $f(x)=U v \phi$ with $\phi \in W_{\frac{3}{2}}(\mathbf{R})$ such that $H \phi=0$ in the distributional sense. Furthermore,

$$
\phi=-G_{0} v f+c_{1} x+c_{2},
$$

where

$$
c_{1}=\frac{\left\langle v_{1}, T_{0} f\right\rangle}{\left\|v_{1}\right\|_{L^{2}(\mathbf{R})}} \quad \text { and } \quad c_{2}=\frac{\left\langle v, T_{0} f\right\rangle}{\|V\|_{L^{1}(\mathbf{R})}}-\frac{\langle x v, v\rangle}{\|V\|_{L^{1}(\mathbf{R})}} c_{1}
$$


with $v_{1}=x v-\frac{\langle x v, v\rangle}{\|V\|_{L^{1}(\mathbf{R})}} v \in\left(Q-S_{0}\right) L^{2}(\mathbf{R})$.

(ii) $f(x) \in S_{2} L^{2}(\mathbf{R})$ if and only if $f(x)=U v \phi$ with $\phi \in W_{\frac{1}{2}}(\mathbf{R})$ such that $H \phi=0$ in the distributional sense. Furthermore,

$$
\phi=-G_{0} v f+\frac{\left\langle v, T_{0} f\right\rangle}{\|V\|_{L^{1}(\mathbf{R})}} .
$$

Proof. (i) Assume that $f \in S_{1} L^{2}(\mathbf{R})$. One has

$$
0=S_{0} T_{0} S_{0} f=S_{0}\left(U+v G_{0} v\right) f .
$$

Note that

$$
0=S_{0}\left(U+v G_{0} v\right) f=(I-\widetilde{P})\left(U+v G_{0} v\right) f=U f+v G_{0} v f-\widetilde{P} T_{0} f,
$$

where $\widetilde{P}$ is the projection onto the $\operatorname{span}\{v, x v\}=\left(S_{0} L^{2}(\mathbf{R})\right)^{\perp}$. In fact, we know that $v$ and $v_{1}=x v-\frac{\langle x v, v\rangle}{\|V\|_{L^{1}(\mathbf{R})}} v$ are the orthogonal basis of $\left(S_{0} L^{2}(\mathbf{R})\right)^{\perp}$. Hence, we have

$$
\begin{aligned}
\widetilde{P} T_{0} f & =\frac{\left\langle v_{1}, T_{0} f\right\rangle}{\left\|v_{1}\right\|_{L^{2}(\mathbf{R})}} v_{1}+\frac{\left\langle v, T_{0} f\right\rangle}{\|V\|_{L^{1}(\mathbf{R})}} v \\
& =x v \frac{\left\langle v_{1}, T_{0} f\right\rangle}{\left\|v_{1}\right\|_{L^{2}(\mathbf{R})}}++v\left(\frac{\left\langle v, T_{0} f\right\rangle}{\|V\|_{L^{1}(\mathbf{R})}}-\frac{\langle x v, v\rangle}{\|V\|_{L^{1}(\mathbf{R})}} \cdot \frac{\left\langle v_{1}, T_{0} f\right\rangle}{\left\|v_{1}\right\|_{L^{2}(\mathbf{R})}}\right) \\
& :=c_{1} x v+c_{2} v .
\end{aligned}
$$

Furthermore, we have

$$
U f=-v G_{0} v f+\widetilde{P} T_{0} f=-v G_{0} v f+c_{1} x v+c_{2} v .
$$

Since $U^{2}=1$, then

$$
f=U^{2} f=U v\left(-G_{0} v f\right)+U\left(c_{1} x v+c_{2} v\right)=U v\left(-G_{0} v f+c_{1} x+c_{2}\right):=U v \phi,
$$

and $v f=v U v \phi=V \phi$. By $G_{0}=\left(\Delta^{2}\right)^{-1}$ and noting that $\Delta^{2}\left(c_{1} x+c_{2}\right)=0$, we have

$$
H \phi=\left(\Delta^{2}+V\right) \phi=\Delta^{2} \phi+V \phi=-v f+V \phi=0 .
$$

Next, we show that $\phi \in W_{\frac{3}{2}}(\mathbf{R})$. It is obvious that $c_{1} x+c_{2} \in W_{\frac{3}{2}}(\mathbf{R})$, so it suffices to show that

$$
G_{0} v f=\frac{1}{12} \int_{\mathbf{R}}|x-y|^{3} v(y) f(y) d y \in W_{\frac{3}{2}}(\mathbf{R}) .
$$

Since $S_{1} \leq S_{0}$, and we know that if $f \in S_{0} L^{2}(\mathbf{R})$, then

$$
\int_{\mathbf{R}} v(y) f(y) d y=0, \text { and } \int_{\mathbf{R}} y v(y) f(y) d y=0 .
$$

Hence

$$
G_{0} v f=\frac{1}{12} \int_{\mathbf{R}}\left(|x-y|^{3}-|x|^{3}+3|x| x \cdot y\right) v(y) f(y) d y:=\frac{1}{12} \int_{\mathbf{R}} K_{1}(x, y) v(y) f(y) d y
$$

with $K_{1}(x, y)=|x-y|^{3}-|x|^{3}+3|x| x \cdot y$.

Indeed, we can divided $K_{1}(x, y)$ into three parts as follows:

$$
\begin{aligned}
K_{1}(x, y)= & |x-y|^{3}-|x|^{3}+3|x| x \cdot y \\
= & \left(x^{2}(|x-y|-|x|)+|x| x \cdot y\right)+y^{2}(|x-y|-|x|) \\
& \quad+\left(y^{2}|x|-2 x \cdot y(|x-y|-|x|)\right) \\
:= & K_{11}(x, y)+K_{12}(x, y)+K_{13}(x, y) .
\end{aligned}
$$


For the first part $K_{11}(x, y)$, we have

$$
\begin{aligned}
K_{11}(x, y) & =\left|\frac{x^{2}\left(y^{2}-2 x y\right)}{|x-y|+|x|}+\frac{x^{3} y+x y|x| \cdot|x-y|}{|x-y|+|x|}\right| \\
& \leq \frac{x^{2} y^{2}}{|x-y|+|x|}+\left|\frac{|x| x y(|x-y|-|x|)}{|x-y|+|x|}\right| \\
& \lesssim|x| y^{2} .
\end{aligned}
$$

Thus, we have

$$
\left|\int_{\mathbf{R}} K_{11}(x, y) v(y) f(y) d y\right| \in W_{\frac{3}{2}}(\mathbf{R}) .
$$

Then, for the second part $K_{22}(x, y)$, we have

$$
\left|K_{12}(x, y)\right|=\left|y^{2}(|x-y|-|x|)\right| \lesssim|y|^{3} .
$$

It is obvious that

$$
\left|\int_{\mathbf{R}} K_{12}(x, y) v(y) f(y) d y\right| \lesssim \int_{\mathbf{R}}|y|^{3} v(y) f(y) d y \in W_{\frac{3}{2}}(\mathbf{R}) .
$$

Finally, for the last part $K_{13}(x, y)$, we have

$$
\left|K_{13}(x, y)\right|=\left|y^{2}\right| x|-2 x y(|x-y|-|x|)| \leq|x| y^{2}+2|x| y^{2} \lesssim|x| y^{2} .
$$

It is easy to know that

$$
\left|\int_{\mathbf{R}} K_{13}(x, y) v(y) f(y) d y\right| \lesssim|x| \int_{\mathbf{R}}|y|^{2} v(y) f(y) d y \in W_{\frac{3}{2}}(\mathbf{R}) .
$$

Hence, we obtain that

$$
\begin{aligned}
\left|G_{0} v f\right|= & \left|\frac{1}{12} \int_{\mathbf{R}} K_{1}(x, y) v(y) f(y) d y\right| \\
\lesssim & \left|\int_{\mathbf{R}} K_{11}(x, y) v(y) f(y) d y\right|+\left|\int_{\mathbf{R}} K_{12}(x, y) v(y) f(y) d y\right| \\
& +\left|\int_{\mathbf{R}} K_{13}(x, y) v(y) f(y) d y\right|
\end{aligned}
$$

which implies that $G_{0} v f \in W_{\frac{3}{2}}(\mathbf{R})$.

On the other hand, let $\phi \in W_{\frac{3}{2}}(\mathbf{R})$ such that $H \phi=0$. We first show that $f=U v \phi \in S_{0} L^{2}(\mathbf{R})$, that is,

$$
\int_{\mathbf{R}} v(y) f(y) d y=0 \text { and } \int_{\mathbf{R}} y v(y) f(y) d y=0 .
$$

Noting that $v f=V \phi=-\Delta^{2} \phi$. Let $\eta(x) \in C_{0}^{\infty}(\mathbf{R})$ such that $\eta(x)=1$ for $|x| \leq 1$ and $\eta(x)=0$ for $|x|>2$. Then for any $\delta>0$, we have

$$
\begin{aligned}
\left|\int_{\mathbf{R}} v(y) f(y) \eta(\delta y) d y\right| & =\left|\int_{\mathbf{R}}\left(\Delta^{2} \phi(y)\right) \eta(\delta y) d y\right|=\left|\int_{\mathbf{R}} \phi(y) \delta^{4} \eta^{(4)}(\delta y) d y\right| \\
& \lesssim \delta^{4}\left\|\langle y\rangle^{-\frac{3}{2}-} \phi(y)\right\|_{L^{2}(\mathbf{R})} \cdot\left\|\langle y\rangle^{\frac{3}{2}+} \eta^{(4)}(\delta y)\right\|_{L^{2}(\mathbf{R})} \\
& \lesssim \delta^{\frac{5}{2}-}\left\|\langle y\rangle^{-\frac{3}{2}-} \phi(y)\right\|_{L^{2}(\mathbf{R})} \cdot\left\|\eta^{(4)}(y)\right\|_{L^{2}(\mathbf{R})} \cdot
\end{aligned}
$$

Taking the limit $\delta \rightarrow 0$ and using the dominated convergence theorem we obtain $\int_{\mathbf{R}} v(y) f(y) d y=0$. Similarly, we have $\int_{\mathbf{R}} v(y) f(y) d y=0$. Thus, $f=U v \phi \in S_{0} L^{2}(\mathbf{R})$. 
Now we turn to prove that $\phi=-G_{0} v f+c_{1} x+c_{2}$. Let $\widetilde{\phi}=\phi+G_{0} v f$, then by assumption and (4.1), $\widetilde{\phi} \in W_{\frac{3}{2}}(\mathbf{R})$ and $\Delta^{2} \widetilde{\phi}=0$, thus $\widetilde{\phi}=\widetilde{c}_{1} x+\widetilde{c}_{2}$. This implies that $\phi=-G_{0} v f+\widetilde{c}_{1} x+\widetilde{c}_{2}$.

Since

$$
\begin{aligned}
0=H \phi=\left[\Delta^{2}+V\right] \phi & =-\left[\Delta^{2}+V\right]\left(G_{0} v f\right)+\left[\Delta^{2}+V\right]\left(\widetilde{c}_{1} x+\widetilde{c}_{2}\right) \\
& =-U v\left(U+v G_{0} v\right) f+U\left(\widetilde{c}_{1} x v^{2}+\widetilde{c}_{2} v^{2}\right) \\
& =U\left(-v T_{0} f+\widetilde{c}_{1} x v^{2}+\widetilde{c}_{2} v^{2}\right),
\end{aligned}
$$

thus we have

$$
\begin{aligned}
\widetilde{c}_{1} x v^{2}+\widetilde{c}_{2} v^{2}=v T_{0} f & \Longrightarrow \widetilde{c}_{1}\langle x v, v\rangle+\widetilde{c}_{2}\langle v, v\rangle=\left\langle v, T_{0} f\right\rangle, \\
\widetilde{c}_{1} x^{2} v^{2}+\widetilde{c}_{2} x v^{2}=x v T_{0} f & \Longrightarrow \widetilde{c}_{1}\langle x v, x v\rangle+\widetilde{c}_{2}\langle x v, v\rangle=\left\langle x v, T_{0} f\right\rangle .
\end{aligned}
$$

By (4.3), we have

$$
\widetilde{c_{2}}=\frac{\left\langle v, T_{0} f\right.}{\|V\|_{L^{1}(\mathbf{R})}}-\widetilde{c}_{1} \frac{\langle x v, v\rangle}{\|V\|_{L^{1}(\mathbf{R})}}
$$

Then, substituting (4.5), $x v=v_{1}+\frac{\langle x v, v\rangle}{\|V\|_{L^{1}(\mathbf{R})}} v$ and $\left\langle v_{1}, v\right\rangle=0$ into (4.4), we have

$$
\widetilde{c}_{1}=\frac{\left\langle v_{1}, T_{0} f\right\rangle}{\left\|v_{1}\right\|_{L^{2}(\mathbf{R})}} \quad \text { and } \quad \widetilde{c}_{2}=\frac{\left\langle v, T_{0} f\right\rangle}{\|V\|_{L^{1}(\mathbf{R})}}-\frac{\langle x v, v\rangle}{\|V\|_{L^{1}(\mathbf{R})}} \widetilde{c}_{1},
$$

which means that $\widetilde{c}_{1}=c_{1}, \widetilde{c}_{2}=c_{2}$. Furthermore, using $f=U v \phi$ and $H \phi=0$, we have

$$
\begin{aligned}
S_{0} T_{0} S_{0} f & =S_{0} T_{0}(U v \phi)=S_{0}\left(U+v G_{0} v\right)(U v \phi)=S_{0}\left(v \phi+v G_{0} v U v \phi\right) \\
& =S_{0} v G_{0}\left(\Delta^{2}+V\right) \phi=S_{0} v G_{0} H \phi=0 .
\end{aligned}
$$

Hence, we obtain that $f \in S_{1} L^{2}(\mathbf{R})$. The proof of Theorem 4.1 (i) is completed.

(ii) Since $S_{2} \leq S_{1}$, by Theorem 4.1 (i), we know that $f \in S_{1} L^{2}(\mathbf{R})$ if and only if $f=U v \phi$ with $\phi \in W_{\frac{3}{2}}(\mathbf{R})$ such that $H \phi=0$ in the distributional sense and

$$
\phi=-G_{0} v f+c_{1} x+c_{2} .
$$

Thus, we just need to prove that $c_{1}=0$ and $\phi \in W_{\frac{1}{2}}(\mathbf{R})$. Indeed, by the proof of Theorem 4.1(i), we know that

$$
v_{1}=x v-\frac{\langle x v, v\rangle}{\|V\|_{L^{1}(\mathbf{R})}} v \in Q L^{2}(\mathbf{R}) .
$$

By Table 1.6, if $f \in S_{2} L^{2}(\mathbf{R})$, then $Q T_{0} f=0$, thus

$$
c_{1}=\left\langle v_{1}, T_{0} f\right\rangle=0 .
$$

Next we show that $\phi \in W_{\frac{1}{2}}(\mathbf{R})$. Since $\phi=-G_{0} v f+\frac{\left\langle v, T_{0} f\right\rangle}{\|V\|_{L^{1}(\mathbf{R})}}$, thus it suffices to show that

$$
G_{0} v f=\frac{1}{12} \int_{\mathbf{R}}|x-y|^{3} v(y) f(y) d y \in W_{\frac{1}{2}}(\mathbf{R}) .
$$

We obtain that if $f \in S_{2} L^{2}(\mathbf{R})$, then

$$
\int_{\mathbf{R}} v(y) f(y) d y=\int_{\mathbf{R}} y v(y) f(y) d y=\int_{\mathbf{R}} y^{2} v(y) f(y) d y=0 .
$$


Thus,

$$
\begin{aligned}
G_{0} v f & =\frac{1}{12} \int_{\mathbf{R}}\left(|x-y|^{3}-|x|^{3}+3|x| x \cdot y-3|x| y^{2}\right) v(y) f(y) d y \\
& :=\frac{1}{12} \int_{\mathbf{R}} K_{2}(x, y) v(y) f(y) d y
\end{aligned}
$$

with $K_{2}(x, y)=|x-y|^{3}-|x|^{3}+3|x| x \cdot y-3|x| y^{2}$.

Indeed, we can divided $K_{2}(x, y)$ into four parts as follows

$$
\begin{aligned}
K_{2}(x, y)= & y^{2}(|x-y|-|x|)-2\left[x y(|x-y|-|x|)+|x| y^{2}\right]+\left[\frac{x^{2} y^{2}}{|x-y|+|x|}-\frac{1}{2}|x| y^{2}\right] \\
& +\left[\frac{|x| x \cdot y(|x-y|-|x|)}{|x-y|+|x|}+\frac{1}{2}|x| y^{2}\right] \\
:= & K_{21}(x, y)+K_{22}(x, y)+K_{23}(x, y)+K_{24}(x, y) .
\end{aligned}
$$

For the first part $K_{21}(x, y)$, we have

$$
\left|K_{21}(x, y)\right|=\left|y^{2}(|x-y|-|x|)\right| \lesssim|y|^{3} .
$$

Thus

$$
\left|\int_{\mathbf{R}} K_{21}(x, y) v(y) f(y) d y\right| \lesssim \int_{\mathbf{R}}|y|^{3}|v(y) f(y)| d y \in W_{\frac{1}{2}}(\mathbf{R}) .
$$

For the second part $K_{22}(x, y)$, we have

$$
\begin{aligned}
\left|K_{22}(x, y)\right| & =2\left|\frac{x \cdot y\left(y^{2}-2 x \cdot y\right)}{|x-y|+|x|}+\frac{|x| y^{2}(|x-y|+|x|)}{|x-y|+|x|}\right| \\
& \lesssim \frac{|x| \cdot|y|^{3}}{|x-y|+|x|}+\left|\frac{|x| y^{2}(|x-y|-|x|)}{|x-y|+|x|}\right| \\
& \lesssim|y|^{3} .
\end{aligned}
$$

Thus

$$
\left|\int_{\mathbf{R}} K_{22}(x, y) v(y) f(y) d y\right| \lesssim \int_{\mathbf{R}}|y|^{3}|v(y) f(y)| d y \in W_{\frac{1}{2}}(\mathbf{R}) .
$$

Then, for the third part $K_{23}(x, y)$, we have

$$
\begin{aligned}
\left|K_{23}(x, y)\right| & =\left|\frac{2 x^{2} y^{2}}{2(|x-y|+|x|)}-\frac{|x| y^{2}(|x-y|+|x|)}{2(|x-y|+|x|)}\right| \\
& =\left|\frac{|x| y^{2} \cdot|(|x-y|-|x|)|}{2(|x-y|+|x|)}\right| \\
& \lesssim|y|^{3} .
\end{aligned}
$$

Thus

$$
\left|\int_{\mathbf{R}} K_{23}(x, y) v(y) f(y) d y\right| \lesssim \int_{\mathbf{R}}|y|^{3}|v(y) f(y)| d y \in W_{\frac{1}{2}}(\mathbf{R}) .
$$

Finally, for the fourth part $K_{24}(x, y)$, we have

$$
\begin{aligned}
\left|K_{24}(x, y)\right| & =\left|\frac{|x| x \cdot y\left(y^{2}-2 x \cdot y\right)}{(|x-y|+|x|)^{2}}+\frac{|x| y^{2}(|x-y|+|x|)^{2}}{2(|x-y|+|x|)^{2}}\right| \\
& \leq \frac{|x| y^{4}}{2(|x-y|+|x|)^{2}}+\left|\frac{x^{2} y^{2}(|x-y|-|x|)}{(|x-y|+|x|)^{2}}\right| \\
& \lesssim|y|^{3} .
\end{aligned}
$$

Thus

$$
\left|\int_{\mathbf{R}} K_{24}(x, y) v(y) f(y) d y\right| \lesssim \int_{\mathbf{R}}|y|^{3} \cdot|v(y) f(y)| d y \in W_{\frac{1}{2}}(\mathbf{R}) .
$$


Hence, we obtain that $G_{0} v f \in W_{\frac{1}{2}}(\mathbf{R})$. Furthermore, we have $\phi \in W_{\frac{1}{2}}(\mathbf{R})$.

On the other hand, let $\phi(x) \in W_{\frac{1}{2}}(\mathbf{R})$ such that $H \phi(x)=0$. Denote $f=U v \phi$, with the similar method of (4.2) and (4.6), we have

$$
\int_{\mathbf{R}} v(y) f(y) d y=0, \int_{\mathbf{R}} y v(y) f(y) d y=0, \int_{\mathbf{R}} y^{2} v(y) f(y) d y=0, S_{0} T_{0} S_{0} f=0,
$$

then we can obtain that $f \in S_{1} L^{2}(\mathbf{R})$ and $G_{0} v f \in W_{\frac{1}{2}}(\mathbf{R})$. Moreover, by Theorem 4.1(i), we know that $\phi=-G_{0} v f+c_{1} x+c_{2}$ since $W_{\frac{1}{2}}(\mathbf{R}) \subset W_{\frac{3}{2}}(\mathbf{R})$. Thus we need that $c_{1} x=\phi+G_{0} v f-c_{2} \in W_{\frac{1}{2}}(\mathbf{R})$ this necessitates that $c_{1}=0$, which implies that $T_{0} f \in \operatorname{span}\{v\}$ and $\phi=-G_{0} v f+\frac{\left\langle v, T_{0} f\right\rangle}{\|V\|_{L^{1}(\mathbf{R})}}$. Hence, we have $f \in S_{2} L^{2}(\mathbf{R})$. The proof of Theorem 4.1 (ii) is completed.

Acknowledgements: A. Soffer is partially supported by the Simon's Foundation (No.395767). Z. Wu and X. H. Yao are partially supported by NSFC (No.11771165 and 12171182). The authors would like to express their sincere gratitude to the reviewing referee for many constructive comments which helped us greatly improve the previous version. We also would like to thank Professor P. Li for his useful discussions, and thank Professor M. Goldberg for his helpful comments and insights for the previous version.

\section{REFERENCES}

[Agm75] S. Agmon, Spectral properties of Schrödinger operators and scattering theory, Ann. Scuola Norm. Sup. Pisa Cl. Sci. (4) 2 (1975), no. 2, 151-218. MR 0397194

[BKS] M. Ben-Artzi, H.Koch, and J.Saut, Dispersion estimates for fourth order Schroödinger equations, C. R. Acad. Sci. Paris Sér. I Math., 330(2000), No.1,87-92. MR 1745182

[BS91] M. Sh. Birman and M. Z. Solomyak, Estimates for the number of negative eigenvalues of the Schrödinger operator and its generalizations, Estimates and asymptotics for discrete spectra of integral and differential equations (Leningrad, 1989-90), Adv. Soviet Math., vol. 7, Amer. Math. Soc., Providence, RI, 1991, pp. 1-55. MR 1306507

[Bou91] J. Bourgain, Global solutions of nonlinear Schrödinger equations. American Mathematical Society Colloquium Publications, 46. American Mathematical Society, Providence, RI, 1999. MR 1691575

[Caz03] Thierry Cazenave, Semilinear Schrödinger equations, Courant Lecture Notes in Mathematics, vol. 10, New York University, Courant Institute of Mathematical Sciences, New York; American Mathematical Society, Providence, RI, 2003. MR 2002047

[CMY12] W. Chen, C. Miao and X. Yao, Dispersive estimates with geometry of finite type, Comm. Partial Differential Equations. 37(2012) 479-510. MR 2889560

[CS01] O. Costin and A. Soffer, Resonance theory for Schrödinger operators, Comm. Math. Phys. 224 (2001), no. 1, 133-152, Dedicated to Joel L. Lebowitz. MR 1868995

[Dav97] E. B. Davies, Limits on $L^{p}$ regularity of self-adjoint elliptic operators, J. Differential Equations 135 (1997), no. 1, 83-102. MR 1434916

[DDY14] Q. Deng, Y. Ding, and X. Yao, Gaussian bounds for higher-order elliptic differential operators with Kato type potentials, J. Funct. Anal. 266 (2014), no. 8, 5377-5397. MR 3177340

[DT79] P. A. Deift and E. Trubowitz, Inverse scattering on the line, Comm. Pure Appl. Math. 32 (1979), no. 2, 121-251. MR 0512420

[DF08] P. D'Ancona and L. Fanelli, Strichartz and smoothing estimates of dispersive equations with magnetic potentials, Comm. Partial Differential Equations 33 (2008), no. 4-6, 1082-1112. MR 2424390

[DH98a] E. B. Davies and A. M. Hinz, Explicit constants for Rellich inequalities in $L_{p}(\Omega)$, Math. Z. 227 (1998), no. 3, 511-523. MR 1612685

[DH98b] E. B. Davies and A. M. Hinz, Kato class potentials for higher order elliptic operators, J. London Math. Soc. (2) 58 (1998), no. 3, 669-678. MR 1678156

[EGT21] M. B. Erdoğan, W. R. Green, and E. Toprak, On the fourth order schrödinger equation in three dimensions: dispersive estimates and zero energy resonance, J. Differential Equations 271 (2021), 152-185. MR 4151179

[EKMT16] I. E. Egorova, E. A. Kopylova, V. A. Marchenko, and G. Teschl, On the sharpening of dispersion estimates for One-Dimensional Schrödinger and Klein-Gordon equations, Russian Math. Surveys 71 (2016), no. 3, 391-415. MR 3535363 
[ES04] M. B. Erdoğan and W. Schlag, Dispersive estimates for Schrödinger operators in the presence of a resonance and/or an eigenvalue at zero energy in dimension three. I, Dyn. Partial Differ. Equ. 1 (2004), no. 4, 359-379. MR 2127577

[ES06] M. B. Erdoğan and W. Schlag, Dispersive estimates for Schrödinger operators in the presence of a resonance and/or an eigenvalue at zero energy in dimension three. II, J. Anal. Math. 99 (2006), 199-248. MR 2279551

MR 687957

[FSY18] H. Feng, A. Soffer, and X. Yao, Decay estimates and Strichartz estimates of fourth-order Schrödinger operator, J. Funct. Anal. 274 (2018), no. 2, 605-658. MR 3724151

[FSWY20] H. Feng, A. Soffer, Z. Wu and X. Yao, Decay estimates for higher-order elliptic operators, Trans. Amer. Math. Soc. 373 (2020), no. 4, 2805-2859. MR 3724151

[FWY18] H. Feng, Z. Wu, and X. Yao, Time asymptotic expansions of solution for fourth-order schrödinger equation with zero resonance or eigenvalue, https://arxiv.org/abs/1812.00223 (2018).

[Gold21] M. Goldberg, In private communication.

[GG15] M. Goldberg and W. R. Green, Dispersive estimates for higher dimensional Schrödinger operators with threshold eigenvalues I: The odd dimensional case, J. Funct. Anal. 269 (2015), no. 3, 633-682. MR 3350725

[GG17] M. Goldberg and W. R. Green, Dispersive estimates for higher dimensional Schrödinger operators with threshold eigenvalues II. The even dimensional case, J. Spectr. Theory 7 (2017), no. 1, 33-86. MR 3629407

[GS04] M. Goldberg and W. Schlag, Dispersive estimates for Schrödinger operators in dimensions one and three, Comm. Math. Phys. 251 (2004), no. 1, 157-178. MR 2096737

[GT19] W. R. Green and E. Toprak, On the fourth order schrödinger equation in four dimensions: dispersive estimates and zero energy resonance, J. Differential Equations 267 (2019), no. 3, 1899-1954. MR 3945621

[GV06] M. Goldberg and M. Visan, A counterexample to dispersive estimates for Schrödinger operators in higher dimensions, Comm. Math. Phys. 266 (2006), no. 1, 211-238. MR 2231971

[Hill20] T. Hill, Dispersive estimates of Schrödinger and Schrödinger-like equations in one dimension, Thesis (Ph.D.)-University of Cincinnati. 2020. MR 4187680

[Hö05] L. Hörmander, The analysis of linear partial differential operators. II, Classics in Mathematics, Springer-Verlag, Berlin, 2005, Differential operators with constant coefficients, Reprint of the 1983 original. MR 2108588

[HS15] I. Herbst and E. Skibsted, Decay of eigenfunctions of elliptic PDE's, I, Adv. Math. 270 (2015), 138180. MR 3286533

[HS17] I. Herbst and E. Skibsted, Decay of eigenfunctions of elliptic PDE's, II, Adv. Math. 306 (2017), 177-199. MR 3581301

[JK79] A. Jensen and T. Kato, Spectral properties of Schrödinger operators and time-decay of the wave functions, Duke Math. J. 46 (1979), no. 3, 583-611. MR 544248

[JN01] A. Jensen and G. Nenciu, A unified approach to resolvent expansions at thresholds, Rev. Math. Phys. 13 (2001), no. 6, 717-754. MR 1841744

[JN04] A. Jensen and G. Nenciu, Erratum: "A unified approach to resolvent expansions at thresholds" [Rev. Math. Phys. 13 (2001), no. 6, 717-754; mr1841744], Rev. Math. Phys. 16 (2004), no. 5, 675-677. MR 2079122

[JSS91] J.-L. Journé, A. Soffer, and C. D. Sogge, Decay estimates for Schrödinger operators, Comm. Pure Appl. Math. 44 (1991), no. 5, 573-604. MR 1105875

[Kat59] T. Kato, Growth properties of solutions of the reduced wave equation with a variable coefficient, Comm. Pure Appl. Math. 12 (1959), 403-425. MR 0108633

[KT98] M. Keel and T. Tao, Endpoint Strichartz estimates, Amer. J. Math. 120 (1998), no. 5, 955-980. MR 1646048

[KT06] H. Koch and D. Tataru, Carleman estimates and absence of embedded eigenvalues, Comm. Math. Phys. 267 (2006), no. 2, 419-449. MR 2252331

[Kur78] S. T. Kuroda, An introduction to scattering theory, Lecture Notes Series, vol. 51, Aarhus Universitet, Matematisk Institut, Aarhus, 1978. MR 528757

[LSY21] P. Li, A. Soffer and X. Yao, Decay Estimates for Fourth-Order Schrödinger Operator in Dimension Two, http://arxiv.org/abs/2110.07154.

[Mou81] E. Mourre, Absence of singular continuous spectrum for certain selfadjoint operators, Comm. Math. Phys. 78 (1980/81), no. 3, 391-408. MR 603501

[Mur82] M. Murata, Asymptotic expansions in time for solutions of Schrödinger-type equations, J. Funct. Anal. 49 (1982), no. 1, 10-56. MR 680855 
[NaSc11] K. Nakanishi, W. Schlag, Invariant manifolds and dispersive Hamiltonian evolution equations. Zurich Lectures in Advanced Mathematics. European Mathematical Society (EMS), Zrich, 2011. MR 2847755

[RS04] I. Rodnianski and W. Schlag, Time decay for solutions of Schrödinger equations with rough and timedependent potentials, Invent. Math. 155 (2004), no. 3, 451-513. MR 2038194

[Sch71] M. Schechter, Spectra of partial differential operators, North-Holland Publishing Co., AmsterdamLondon; American Elsevier Publishing Co., Inc., New York, 1971, North-Holland Series in Applied Mathematics and Mechanics, Vol. 14. MR 0447834

[Sch05] W. Schlag, Dispersive estimates for Schrödinger operators in dimension two, Comm. Math. Phys. 257 (2005), no. 1, 87-117. MR 2163570

[Sch07] W. Schlag, Dispersive estimates for Schrödinger operators: a survey, Mathematical aspects of nonlinear dispersive equations, Ann. of Math. Stud., vol. 163, Princeton Univ. Press, Princeton, NJ, 2007, pp. 255-285. MR 2333215

[Sch20] W. Schlag, On pointwise decay of waves, J. Math. Phys. 62 (2021), no. 6, Paper No. 061509,27 pp. MR 4276283

[Sim18a] B. Simon, Tosio Kato's work on non-relativistic quantum mechanics: part 1, Bull. Math. Sci. 8 (2018), no. 1, 121-232. MR 3775269

[Sim18b] B. Simon, Tosio Kato's work on non-relativistic quantum mechanics: part 2, Bull. Math. Sci. (2018), In Press.

[Ste93] E. M. Stein, Harmonic analysis: real-variable methods, orthogonality, and oscillatory integrals, Princeton Mathematical Series, vol. 43, Princeton University Press, Princeton, NJ, 1993, With the assistance of Timothy S. Murphy, Monographs in Harmonic Analysis, III. MR 1232192

[SYY18] A. Sikora, L. Yan, and X. Yao, Spectral multipliers, Bochner-Riesz means and uniform Sobolev inequalities for elliptic operators, Int. Math. Res. Not. IMRN (2018), no. 10, 3070-3121. MR 3805196

[Tao06] T. Tao, Nonlinear dispersive equations, CBMS Regional Conference Series in Mathematics, vol. 106, Published for the Conference Board of the Mathematical Sciences, Washington, DC; by the American Mathematical Society, Providence, RI, 2006, Local and global analysis. MR 2233925

[Wed99] R. A. Weder, The $W^{k, p}$ - continuity of the Schrödinger wave operatos on the line, Comm. Math. Phys. 208 (1999), no. 2, 507-520. MR 1729096

[Wed00] R. A. Weder, $L^{p}-L^{p^{\prime}}$ estimates for the Schrödinger equation on the line and inverse scattering for the non linear Schrödinger equation with a potential, J. Funct. Analysis 170(2000), 37-68.

[Yaj95] K. Yajima, The $W^{k, p}$-continuity of wave operators for Schrödinger operators, J. Math. Soc. Japan 47 (1995), no. 3, 551-581. MR 1331331

Avy Soffer, Mathematics Department, Rutgers University, New Brunswick, NJ, 08903, USA

Email address: soffer@math.rutgers.edu

Zhao Wu, School of Mathematics and Statistics, Central China Normal University, Wuhan, 430079, P.R. China

Email address: wuzhao218@yahoo.com

Xiaohua Yao, Department of Mathematics and Hubei Province Key Laboratory of Mathematical Physics, Central China Normal University, Wuhan, 430079, P.R. China

Email address: yaoxiaohua@mail.ccnu.edu.cn 